\title{
Application of GAP (Groups, Algorithms and Programming) System to Stereoisograms for Characterizing $R S$-Stereoisomers of Cubane Derivatives
}

\author{
Shinsaku Fujita \\ Shonan Institute of Chemoinformatics and Mathematical Chemistry, \\ Kaneko 479-7 Ooimachi, Ashigara-Kami-Gun, Kanagawa-Ken, \\ 258-0019 Japan \\ shinsaku_fujita@nifty.com
}

(Received September 10, 2021)

\begin{abstract}
The PCI (Partial-Cycle-Index) method of Fujita's USCI (Unit-Subduced-CycleIndex) approach has been applied to symmetry-itemized enumerations of cubane derivatives, where groups for specifying three-aspects of symmetry, i.e., the point group $\mathbf{O}_{h}$ for chirality/achirality, the $R S$-stereogenic group $\mathbf{O}_{\tilde{\sigma}}$ for $R S$-stereogenicity/ $R S$-astereogenicity, and the $L R$-permutation group $\mathbf{O}_{\widehat{I}}$ for sclerality/ascrelarity are considered as the subgroups of the $R S$-stereoisomeric group $\mathbf{O}_{h \widetilde{\sigma} \widehat{I}}$. Five types of stereoisograms are adopted as diagrammatical expressions of $\mathbf{O}_{h \widetilde{\sigma} \widehat{I}}$, after combinedpermutation representations (CPR) are created as new tools for treating various groups according to Fujita's stereoisogram approach. The use of CPRs under the GAP (Groups, Algorithms and Programming) system has provided new GAP functions for promoting symmetry-itemized enumerations. The type indices for characterizing stereoisograms (e.g., $[a,-,-]$ for a type-V stereoisogram) have been sophisticated into $R S$-stereoisomeric indices (e.g., $\left[\left[\mathbf{C}_{s}^{\prime}, \widetilde{\mathbf{C}}_{s}^{\prime}, \mathbf{C}_{1}\right]\right]$ for a cubane derivative with the composition $\left.\mathrm{H}_{5} \mathrm{Ap} \overline{\mathrm{p}}\right)$. The type- $\mathrm{V}$ stereoisograms for cubane derivatives with the composition $\mathrm{H}_{5} \mathrm{Ap} \overline{\mathrm{p}}$ are discussed under extended pseudoasymmetry as a new concept.
\end{abstract}




\section{Introduction}

Through the history of stereochemistry, two different ways taken by van't Hoff (asymmetry) $[1,2]$ and by Le Bel (dissymmetry) [3,4] have caused serious conceptual confusions continuously, as discussed detailedly [5]. Although the van't Hoff's term "asymmetry" was replaced by the term "stereogenicity" in the article by Mislow-Siegel [6], while the Le Bel's term "dissymmetry" was replaced by the term "chirality" in the lecture by Lord Kelvin [7], the serious conceptual confusions described above have not been overcome. Mislow-Siegel's "stereogenicity" [6] has been found to be an ad hoc remedy without mathematical formulations. Thus, Mislow-Siegel's "stereogenicity" with the use of the term "chirotopicity" has enhanced exceptional treatment of "pseudoasymmetry", so as to increase the confusion due to permutation groups without differentiating reflections from rotations.

Modern stereochemistry lays stress on van't Hoff's way (asymmetry and later stereogenicity) as if Le Bel's way (dissymmetry and later chirality) is involved subsidiarily in van't Hoff's way. This methodology results in the misleading attitude that the conclusion due to van't Hoff's way (asymmetry and later stereogenicity) is discussed from the aspect of chirality. Even if their target molecules are chiral, for example, experimental approaches (e.g., catalytic asymmetric synthesis by Ryoji Noyori (Nobel-Prize 2001)) are based on van't Hoff's way (asymmetry and later stereogenicity) as far as they are interested in processes of stereospecific syntheses. Moreover, the chirality of each target molecule is specified by means of $R / S$-stereodescriptors, which stem from the CIP (Cahn-Ingold-Prelog) system by Vladimir Prelog (Nobel-Prize 1975) et al. Note that although such $R / S$-stereodescriptors rely mainly on stereogenicity proposed by Mislow and Siegel [6] (not chirality, nor $R S$-stereogenicity), they aim at specifying chirality without integration between misleading attitude concerning stereogenicity and chirality.

In order to discuss the net interaction between chirality and stereogenicity, the author (Fujita) has proposed the concept of $R S$-stereogenicity [8,9], which is a substantial and meaningful restriction of Mislow-Siegel's stereogenicity [6]. Fujita's $R S$-stereogenicity is differentiated from Mislow-Siegel's stereogenicity by considering five types of stereoisograms. In a previous account article [10], the author (Fujita) has pointed out two aspects of symmetry, i.e., chirality and $R S$-stereogenicity, as two kinds of handedness. After he has proposed sclerality as an additional aspect, he has accomplished the Aufheben of the 
three aspects so as to propose the concept of $R S$-stereoisomerism. Group-theoretically speaking, a pair of chirality/achirality is discussed on the basis of point groups; a pair of $R S$-stereogenicity/RS-astereogenicity is discussed on the basis of $R S$-stereogenic groups; and a pair of sclerality/asclerality is discussed on the basis of LR (ligand-reflection) permutation groups; and finally $R S$-stereoisomerism as the integrated concept is discussed on the basis of $R S$-stereoisomeric groups [8,9,11].

The applicabilities of Fujita's proligand method for gross enumeration [12] have been studied by using a cubane skeleton of $\mathbf{O}_{h}$-point group as a probe; i.e., gross enumerations of cubane derivatives by Fujita's proligand method [13], by Fujita's markaracter method [14], by Fujita's characteristic-monomial method [15], by Fujita's extended-superposition method [16], and by Fujita's double-coset-representation method [17].

Fujita's USCI (unit-subduced-cycle-index) approach is based on the concepts of subduction of coset representations, sphericities, and chirality fittingness, which are integrated to develop the concept of unit subduced cycle indices without and with chirality fittingness (USCIs and USCI-CFs) [18]. Fujita's USCI approach [18, 19] have been applied to symmetry-itemized enumerations of cubane derivatives by the partial-cycle-index method [20] and the elementary-superposition method of Fujita's USCI approach [21]; as well as enumeration due to the edge strategy [22]. Systematic enumeration and symmetries of cubane derivatives have been discussed in a review article [23]. Fujita's stereoisogram approach $[24,25]$ has been applied to discuss stereoisograms of cubane derivatives by using an $R S$-stereoisomeric group [26].

Remaining tasks are the examination of applicability of newly-defined combinedpermutation representations (CPRs) [27] to Fujita's stereoisogram approach under the GAP (Groups, Algorithms and Programming) system, [28] as well as further feasibility of Fujita's stereoisogram approach to studies concerning symmetry-itemized enumeration of $R S$-stereoisomers.

\section{Construction of stereoisograms for cubane deriva- tives}

\subsection{Elementary stereoisogram for a cubane skeleton}

Although stereoisograms of cubane derivatives have been studied by using an $R S$-stereoisomeric group [26], they are now reinvestigated by using newly-defined combined-permu- 
tation representations (CPRs) [27] under the GAP system [28]. Note that early enumerations of Fujita's stereoisogram approach [26] under the $R S$-stereoisomeric group $\mathbf{O}_{h \widetilde{\sigma} \widehat{I}}$ are conducted without adopting CPRs by using the Maple programming system as the continuation of Fujita's USCI approach [20].

Essences of the previous report [26] are repeated by using CPRs as a new matter. Fujita's stereoisogram approach adopts the concept of proligand-promolecule model based on a given skeleton, where a proligand is presumed to be an abstract ligand (substituent) with chirality/achirality but with no concrete 3D information, and a promolecule is regarded as an abstract molecule which is formed from a skeleton and several proligands. During this process of forming a promolecule, the symmetry of a skeleton (e.g., the point group $\mathbf{O}_{h}$ for a cubane skeleton) is restricted into an appropriate subgroup of $\mathbf{O}_{h}$ to specify the point-group symmetry of the resulting promolecule. Fujita's stereoisogram approach presumes that such a skeleton is governed by an $R S$-stereoisomeric group (e.g., $\mathbf{O}_{h \widetilde{\sigma} \widehat{I}}$ for a cubane skeleton), which is an extention of the point group $\mathbf{O}_{h}$. According to the restriction of the point group $\mathbf{O}_{h}$, the corresponding $R S$-stereoisomeric group $\mathbf{O}_{h \widetilde{\sigma} \widehat{I}}$ is restricted to its subgroup to specify the resulting promolecule.

Figure 1 illustrates an elementary stereoisogram for a cubane skeleton. The skeleton 1 (A) is selected as a reference skeleton for indicating a cubane skeleton, which is paired with an enantiomeric skeleton $\overline{\mathbf{1}}(\mathrm{B})$. The pair of enantiomers $\left[\begin{array}{ll}\mathbf{1} & \overline{\mathbf{1}}\end{array}\right]$ belongs to the point group $\mathbf{O}_{h}$.

\subsection{Chiral point group $\mathrm{O}$ as a fundamental group}

The skeleton 1 (A) is stabilized by the point group $\mathbf{O}$ (0_cube: order 24), which is composed by 24 rotations generated from a set of generators gen_1 by using a GAP function Group as follows. The order of $\mathbf{O}$ (O_cube) is obtained by using a GAP function Size and the 24 rotations (as permutations) are obtained by a GAP function Elements.

\section{Point Group O (0_cube: order 24)}

gap> \#Point group 0

gap> gen_1 := $[(1,2,3,4)(5,6,7,8),(2,4,5)(3,8,6)] ;$;

gap> 0_cube := Group (gen_1); \#cube-vertical

Group $([(1,2,3,4)(5,6,7,8),(2,4,5)(3,8,6)])$

gap> Size (0_cube):

24

gap> Display (Elements (0_cube));

$[(),(2,4,5)(3,8,6),(2,5,4)(3,6,8),(1,2)(3,5)(4,6)(7,8),(1,2,3,4)(5,6,7,8)$,

$(1,2,6,5)(3,7,8,4),(1,3,6)(4,7,5),(1,3)(2,4)(5,7)(6,8),(1,3,8)(2,7,5)$,

$(1,4,3,2)(5,8,7,6),(1,4,8,5)(2,3,7,6),(1,4)(2,8)(3,5)(6,7),(1,5,6,2)(3,4,8,7)$, 


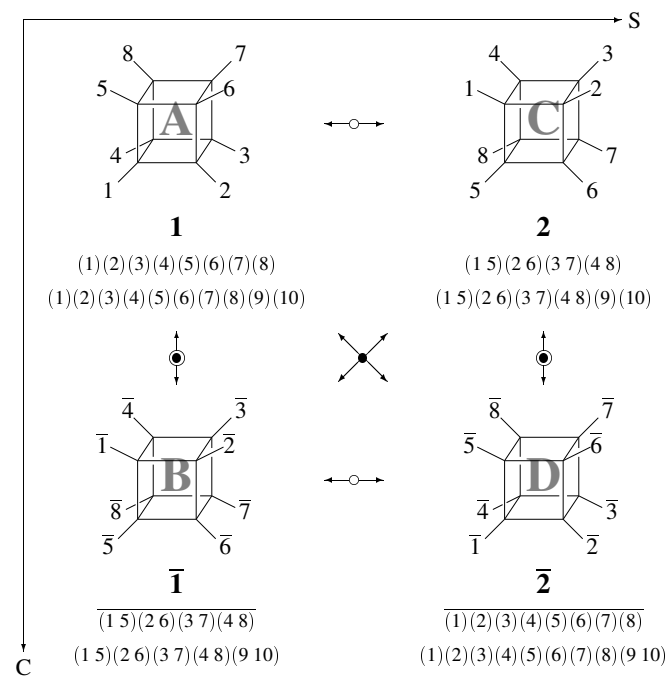

Figure 1. Elementary stereoisogram for a cubane skeleton. The respective skeletons are tentatively linked with double-headed arrows [26].

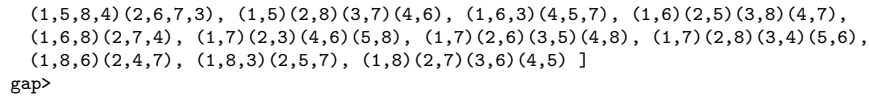

The mark table (tom_O_cube) of the point group $\mathbf{O}$ (0_cube) is obtained by using the GAP function Table0fMarks as a $11 \times 11$ lower-triangular matrix as follows:

Mark Table of Point Group O (0_cube: order 24)

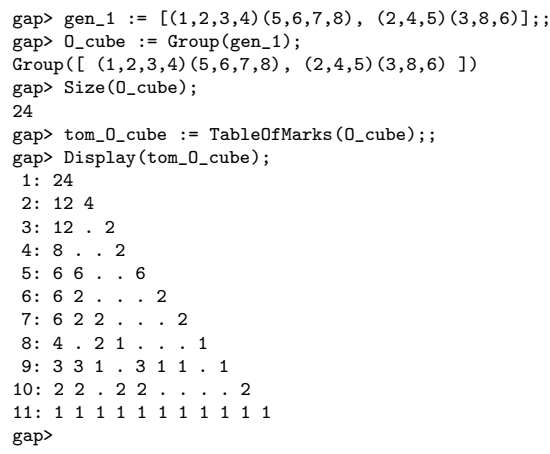

This mark table tom_0_cube is not standardized (cf. Ref. [29]) so that the sequence of the subgroups obey the convention of the GAP system, which is shown above each 
upperbrace of the following $\mathrm{SSG}_{\mathbf{O}}$. It should be noted that the use of Size is neccessary to obtain a mark table tom_0_cube with a settled sequence.

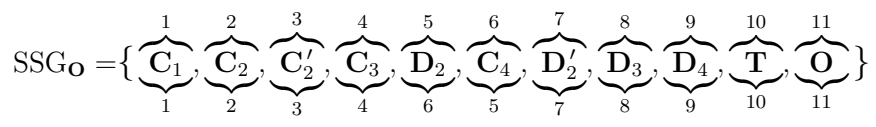

The author (Fujita) has calculated the following standard mark table by means of the FORTRAN77 calculation [20,21]. The mark table tom_0_cube calculated above by means of the GAP function Table0fMarks should be sorted to convert into the standard mark table shown below. In particular, tom_0_cube has the sequence $\overbrace{\underbrace{\mathbf{D}_{2}}_{6}}^{5} \overbrace{\mathbf{C}_{4}}^{6} \overbrace{\underbrace{\mathbf{D}_{2}^{\prime}}_{7}}^{7}$ according to upper sequential numbers, which should be sorted into the standard sequence $\overbrace{\underbrace{C_{4}}_{5}}^{\overbrace{\underbrace{5}_{D_{2}}}^{5}}$ $\overbrace{\underbrace{\mathbf{D}_{2}^{\prime}}_{7}}^{7}$ according to lower sequential numbers. As a result of this sorting, there emerges the following standard mark table, which has originally been constructed by the subduction procedure based on the FORTRAN77 programming language.

\section{Standard Mark Table for the Point Group O (O_cube)}

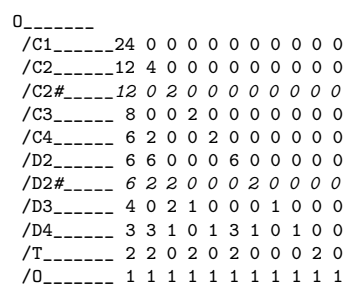

The upper sequence of Eq. 1 is reprented by list1, while the lower sequence is represented by list2. The resulting permutation is obtained by the GAP function PermListList to be perm $=(5,6)$. This is applied to the unsorted mark table tom_0_cube. Thereby, the resulting sorted mark table is identical with the standard mark table shown above obtained by the FORTRAN77 language.

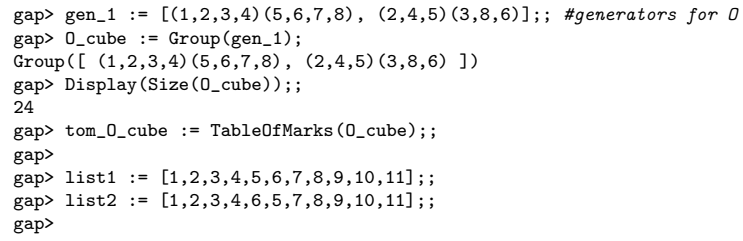




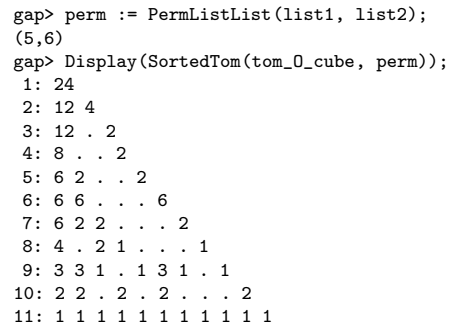

Such standardizations of mark tables and USCI-CF (unit subduced cycle indices with chirality fittingness) tables have been discussed by using different $\mathbf{O}_{h}$-skeletons (e.g., octahedron (OC-6), cube (CU-8), cuboctahedron, truncated octahedron, and truncated hexahedron) as probes [30] after various GAP functions were newly developed to carry out concordant generation of standard mark tables and USCI tables [29].

\subsection{Point group $\mathrm{O}_{h}$ for the first-kind of handedness}

A pair of enantiomeric skeletons, $\mathbf{1}(\mathrm{A})$ and $\mathbf{\mathbf { 1 }}(\mathrm{B})$, is controlled by the point group $\mathbf{O}_{h}$ (Oh_cube: order 48) which is composed of the 24 rotations $(\in \mathbf{O})$ and additional 24 reflections generated from a set of generators gen_2. The reflection $\sigma_{h(1)}$ is illustrated in Figure 2, where the interconversion of a pair of enantiomers $\mathbf{1} \overline{\mathbf{1}}$ is controlled by a CPR (combined-permutation representation). In the CPR, an overline for a mirrorpermutation (for the Maple programming language [20]) is replaced by an additional 2-cycle permutation (9 10) (for the GAP system in this article).

\section{Point Group $\mathbf{O}_{h}$ (Oh_cube: order 48)}

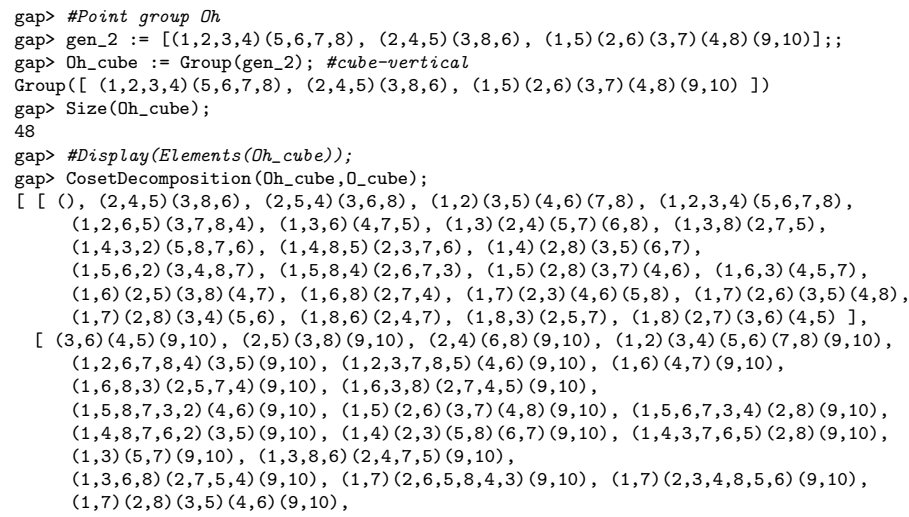


The resulting group $\mathbf{O}_{h}$ (Oh_cube: order 48) is decomposed into two cosets by using the GAP function CosetDecomposition. The 24 rotations of the first coset (surrounded by the first inner pair of square brackets) belong to its maximum chiral subgroup $\mathbf{O}$ (order 24) and the 24 reflections of the second coset (surrounded by the second inner pair of square brackets) belongs to the coset $\mathbf{O} \sigma_{h(1)}$ according to the following coset decomposition:

$$
\begin{aligned}
\mathrm{O}_{h} & =\underbrace{\mathrm{O}}_{\mathrm{A}}+\underbrace{\mathrm{O} \sigma}_{\mathrm{B} \sigma} \\
& =\underbrace{\mathrm{O}}_{\mathrm{B}}+\underbrace{\mathrm{O} i}
\end{aligned}
$$

where the symbol $\sigma$ represents an appropriate reflection (e.g., $\left.\sigma_{h(1)}\right)$. Note that the inversion $i$ is regarded as a kind of reflection, so that the coset $\mathbf{O} \sigma$ is identical with the coset $\mathbf{O} i$. The resulting coset for reflections, i.e., $\underbrace{\mathbf{O} \sigma}_{\mathrm{B}}$ (or $\underbrace{\mathbf{O} i}_{\mathrm{B}})$, is composed of permutations with an additional 2-cycle permutation ( $\left.\begin{array}{ll}9 & 10\end{array}\right)$.

\section{$2.4 R S$-stereogenic group $\mathrm{O}_{\widetilde{\sigma}}$ for the second-kind of handedness}

The horizontal direction of the elementary stereoisogram (Figure 1) indicates $R S$-stereogenicity, where the two skeletons, i.e., $\mathbf{1}(\mathrm{A})$ and $\mathbf{2}(\mathrm{C})$, construct a pair of $R S$-diastereomers, which are interchanged by an $R S$-permutation $\widetilde{\sigma}_{h(1)}(\sim(15)(26)(37)(48)(1)(2) \in \mathbf{O} \widetilde{\sigma})$. The effect of the $R S$-permutation $\widetilde{\sigma}_{h(1)}$ onto the cubane skeleton $\mathbf{1}$ is depicted in Figure 3. If we obey the conventions of the GAP system, any 1-cycle can be omitted, so that we can use $(15)(26)(37)(48)$ after the deletion of an overline from $\overline{(15)(26)(37)(48)}$ or the deletion of two 1-cycles (1)(2) from (1 5)(2 6)(3 7)(4 8)(9)(10), which is generated from a 2-cycle $(9$ 10). The resulting numbered skeleton 2 is not accompanied with ligand reflections, so that the eight positions are numbered without using an overbar.

The effect of $\widetilde{\sigma}_{h(1)}$ means the detachment of substituents from one skeleton $\mathbf{1}(\mathrm{A})$ and the reattachment of the detached substituents, so as to give the other skeleton $\mathbf{2}(\mathrm{C})$. No inversion in each substituent occurs during the detachment-reattachment process shown in Figure 3, so that no overline is attached in each position of the resulting skeleton 2 (C). The detachment-reattachment process (Figure 3) can be alternatively considered to be the intervention of such a graph as $\mathbf{3}$, where the inner square of $\mathbf{3}$ moves upwards or downwards to form a cubane skeleton. 

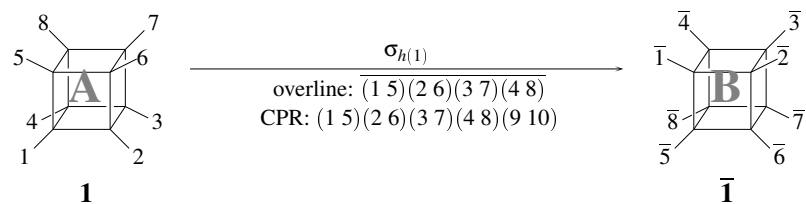

Figure 2. Effects of a reflection for interconversion of a pair of enantiomers $\mathbf{1} / \mathbf{1}$. CPR (combined-permutation representation) is used for representing a reflection operation, where an overline is replaced by a permutation (9 10).

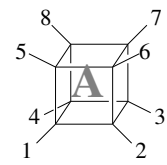

1
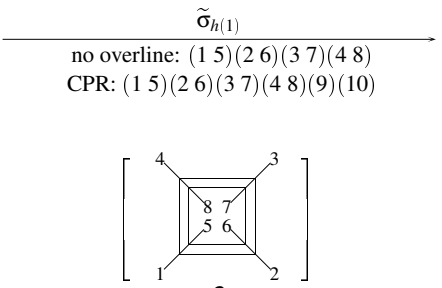

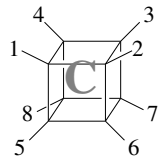

2

3

Figure 3. Effects of an $R S$-permutation for interconversion of a pair of $R S$ dastereomers $\mathbf{1} / \mathbf{2}$ as well as its Interpretation via a graph $\mathbf{3}$.

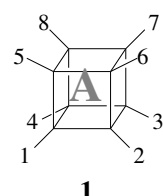

1

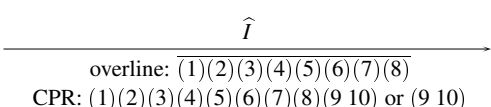

CPR: $(1)(2)(3)(4)(5)(6)(7)(8)(910)$ or $(910)$

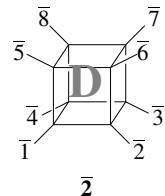

$\overline{\mathbf{2}}$

Figure 4. Effects of a LR (ligand-reflection) permuation for interconversion of a pair of holantimers $\mathbf{1} / \overline{\mathbf{2}}$. 
Group-theoretically speaking, the horizontal directions of the elementary stereoisogram are governed by an $R S$-stereogenic group $\mathbf{O}_{\widetilde{\sigma}}$ (Os_cube), which is generated by a set of generators gen_3 as follows:

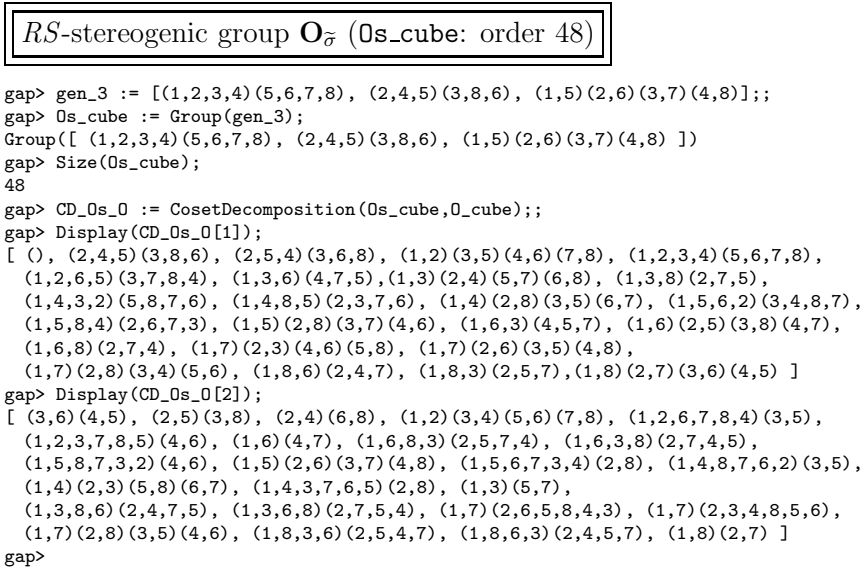

The coset decomposition CD_0s_0, which is generated by means of the GAP function CosetDecomposition, is separated into two cosets, where the second coset CD_Os_0[2] $(\mathbf{O} \widetilde{\sigma}: \mathrm{C})$ is generated by omitting ligand-reflections $(910)$ from the reflections collected in the coset $\mathbf{O} \sigma(\mathrm{B})$ of Eq. 2. They are collected as $\mathbf{O} \widetilde{\sigma}(\mathrm{C})$ of Eq. 3:

$$
\begin{aligned}
\mathrm{O}_{\tilde{\sigma}} & =\underbrace{\mathrm{O}}_{\mathrm{A}}+\underbrace{\mathrm{O}_{\tilde{\sigma}}}_{\mathrm{C}} \\
& =\underbrace{\mathrm{O}}_{\mathrm{A}}+\underbrace{\mathrm{O}_{\tilde{\tau}}}_{\mathrm{C}}
\end{aligned}
$$

\section{$2.5 \quad L R$-permutation group $\mathrm{O}_{\widehat{I}}$ as a key group for integrating two-kinds of handedness}

Let us examine the diagonal directions of the elementary stereoisogram (Figure 1). At first, the relationship between $\overline{\mathbf{1}}$ (Figure 2) and $\mathbf{2}$ (Figure 3) indicates the following relationship:

$$
\widetilde{\sigma}_{h(1)} \sigma_{h(1)}^{-1}=\widehat{I} \sim(1)(2)(3)(4)(5)(6)(7)(8)(910)=(910)
$$

It follows that the action of $(9$ 10) $(=\widehat{I})$ on $\overline{\mathbf{1}}$ generates $\mathbf{2}$ vice versa along one of the diagonal directions. In a parallel way, the action of $(9$ 10) $(=\widehat{I})$ on $\mathbf{1}$ generates $\overline{\mathbf{2}}$ along 
the other one of diagonal directions. Because $\overline{\mathbf{2}}$ is enantiomeric to $\mathbf{2}$ (Compare Figure 3 and Figure 4), the relationship between $\mathbf{1}$ and $\overline{\mathbf{2}}$ (or between $\overline{\mathbf{1}}$ and $\mathbf{2}$ ) is called $a$ holantimeric relationship. Note that the CPR $(1)(2)(3)(4)(5)(6)(7)(8)(910)$ or $(910)$ is used to designate $\widehat{I}$ in place of the overlined expression, $\overline{(1)(2)(3)(4)(5)(6)(7)(8)}$.

Group-theoretically speaking, such a holantimeric pair $\mathbf{1}$ and $\overline{\mathbf{2}}$ along the one of the diagonal direction is governed by an $L R$-permutation group $\mathbf{O}_{\widehat{I}}$. A set of generators gen_4 generates the $L R$-permutation group $\mathbf{O}_{\widehat{I}}$ (OI_cube) as follows:

\section{$L R$-permutation group $\mathbf{O}_{\widehat{I}}\left(\mathrm{OI}_{-}\right.$cube: order 48$)$}

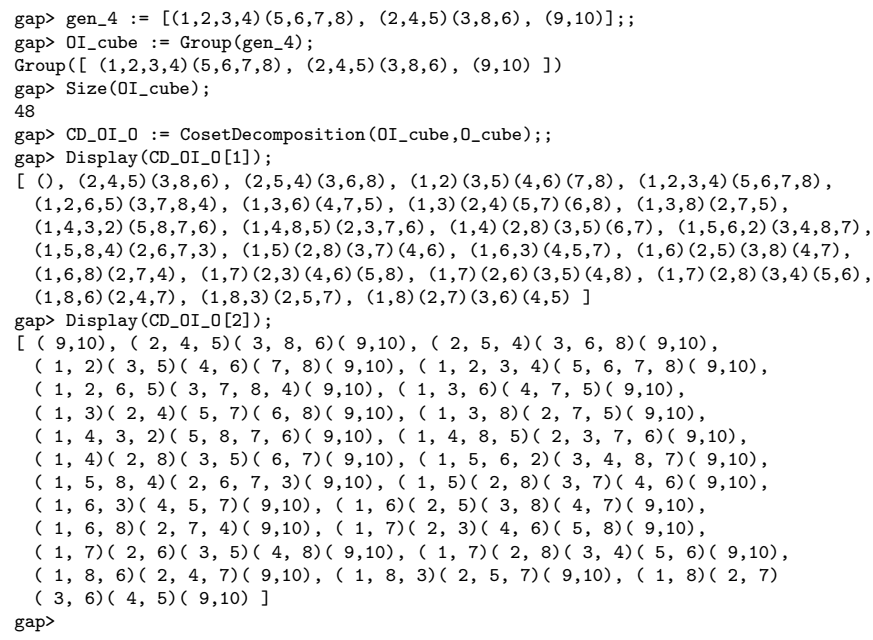

The coset decomposition CD_OI_O, which is generated by means of the GAP function CosetDecomposition, is separated into two cosets, where the second coset CD_OI_O[2] $(\mathbf{O} \widehat{I}$ : D) is generated by operating an $L R$-permutation (9 10 ) additionally onto the operations of $\mathbf{O}$ (the first coset CD_OI_O[1]).

This process can be expressed by an usual mathematical expression. Thus, the second coset $\mathbf{O} \widehat{I}$ (CD_OI_O[2]) can be constructed by starting the proper rotations contained in $\mathbf{O}$. The resulting elements contained in $\mathbf{O} \widehat{I}$ are called ligand-reflections, where their symbols are attached by hat accents. As a result, the $L R$-permutation group $\mathbf{O}_{\widehat{I}}$ (order 48) is constructed as follows:

$$
\mathbf{O}_{\widehat{I}}=\underbrace{\mathrm{O}}_{\mathrm{A}}+\underbrace{\mathrm{O} \widehat{I}}_{\mathrm{D}}
$$

where the representative element $\widehat{I}$ can be replaced by an arbitrary $L R$-permutation in- 
cluded in the coset $\mathbf{O} \widehat{I}$ (CD_OI_O[2]).

\subsection{The $R S$-stereoisomeric group $\mathrm{O}_{h \widetilde{\sigma} \widehat{I}}$ for integrating two-kinds of handedness}

The $R S$-stereoisomeric group $\mathbf{O}_{h \widetilde{\sigma} \widehat{I}}$ is generated from a set of generators gen_5 by means of the GAP function Group as follows. The GAP function CosetDecomposition is used to obtain the coset decomposition of the group $\mathbf{O}_{h \widetilde{\sigma} \widehat{I}}$ by $\mathbf{O}$ (CD_OhsI_0). Each coset is obtained by inputting CD_OhsI_O[1] to CD_OhsI_O[4]. The results are consistent with Eq. 6.

The $R S$-stereoisomeric group $\mathbf{O}_{h \widetilde{\sigma} \widehat{I}}($ Ohs I_cube: order 96)

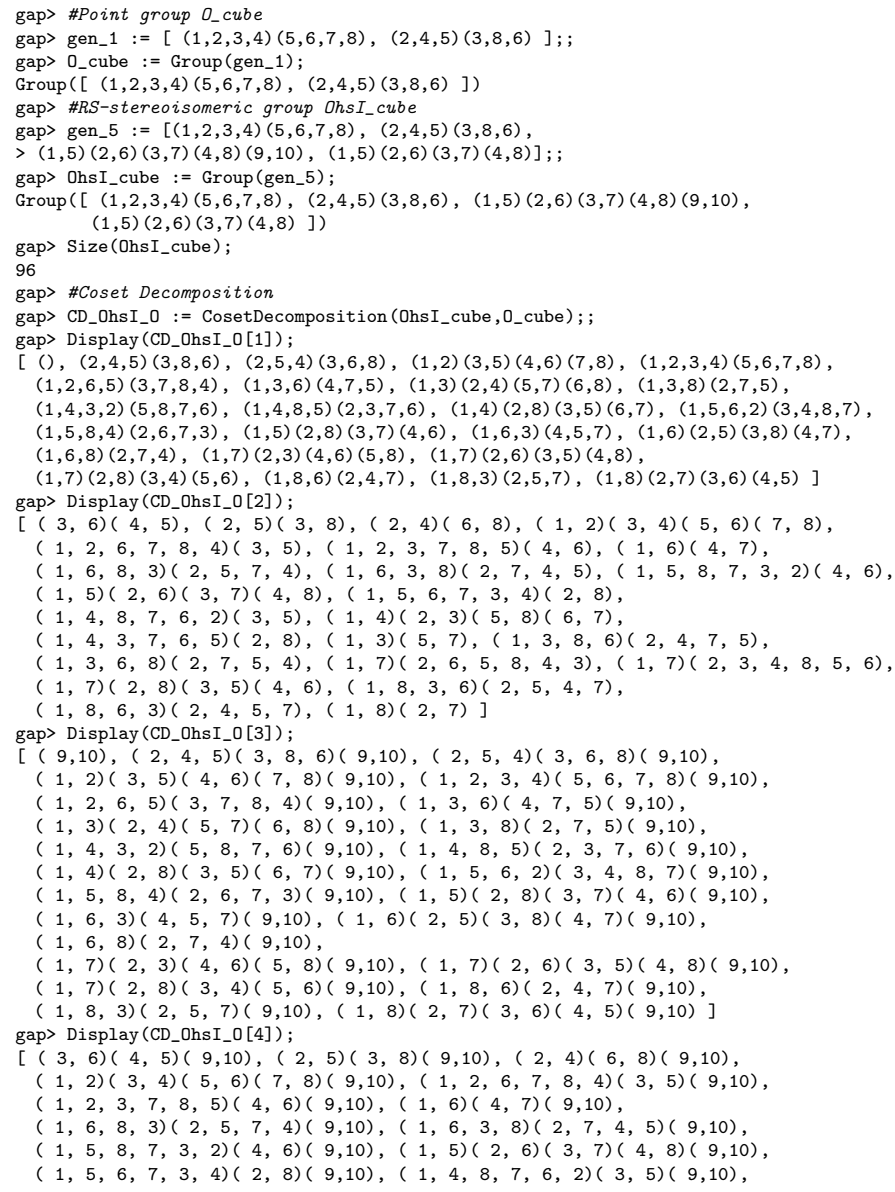


$(1,4)(2,3)(5,8)(6,7)(9,10),(1,4,3,7,6,5)(2,8)(9,10)$,

$(1,3)(5,7)(9,10),(1,3,8,6)(2,4,7,5)(9,10)$,

$(1,3,6,8)(2,7,5,4)(9,10),(1,7)(2,6,5,8,4,3)(9,10)$,

$(1,7)(2,3,4,8,5,6)(9,10),(1,7)(2,8)(3,5)(4,6)(9,10)$,

$(1,8,3,6)(2,5,4,7)(9,10)$,

$(1,8,6,3)(2,4,5,7)(9,10),(1,8)(2,7)(9,10)]$ gap>

The element ()$(\sim I)$ in the first coset CD_OhsI_O [1] corresponds to the reference skeleton 1 (A) shown in Figure 1; the element $(1,5)(2,6)(3,7)(4,8)(\sim \widetilde{\sigma})$ in the second coset CD_OhsI_O[2] corresponds to the $R S$-diasteromeric skeleton $2(\mathrm{C})$; the element $(9,10)$ $(\sim \widehat{I})$ in the third coset CD_OhsI_O[3] corresponds to the holantimeric skeleton $\overline{2}(\mathrm{D})$; and finally the element $(1,5)(2,6)(3,7)(4,8)(9,10)(\sim \sigma)$ in the fourth coset CD_OhsI_0 [4] corresponds to the enantiomeric skeleton $\overline{\mathbf{1}}(\mathrm{B})$. The assignment of the symbols $\mathrm{A}$ to $\mathrm{D}$ are consistent to the assignment shown in Eq. 6.

Figure 1 shows an elementary stereoisogram for a cubane skeleton, from which we are able to depict stereoisograms for characterizing the $R S$-stereoisomeric group $\mathbf{O}_{h \widetilde{\sigma} \widehat{I}}$. A skeleton having an appropriate numbering such as $\mathbf{1}$ is selected as a reference skeleton. Then, a mirror-numbered skeleton $\overline{\mathbf{1}}$, an $R S$-diastereomeric skeleton $\mathbf{2}$, and a ligandreflection (LR) skeleton $\overline{\mathbf{2}}$ are depicted according to the following coset decomposition of the $R S$-stereoisomeric group $\mathbf{O}_{h \widetilde{\sigma} \hat{I}}$ by the $\mathbf{O}$-subgroup:

$$
\begin{aligned}
\mathrm{O}_{h \widetilde{\sigma} \widehat{I}} & =\underbrace{\mathrm{O}}_{\mathrm{A}}+\underbrace{\mathrm{O} \sigma}_{\mathrm{B}}+\underbrace{\mathrm{O} \widetilde{\sigma}}_{\mathrm{C}}+\underbrace{\mathrm{O} \hat{I}}_{\mathrm{D}} \\
& =\underbrace{\mathrm{O}}_{\mathrm{A}}+\underbrace{\mathrm{O} i}_{\mathrm{B}}+\underbrace{\mathrm{O} \tilde{\imath}}_{\mathrm{C}}+\underbrace{\mathrm{O} \widehat{I}}_{\mathrm{D}},
\end{aligned}
$$

\section{Symmetry-itemized enumeration of cubane deriva- tives}

\subsection{Partial cycle indices with chirality fittingness (PCI-CFs) un- der the point group $\mathrm{O}_{h}$}

The point group $\mathbf{O}_{h}$ (order 48) has 33 subgroups up to conjugacy, which have been discussed in terms of a non-redundant set of subgroups (SSG) [31]: 


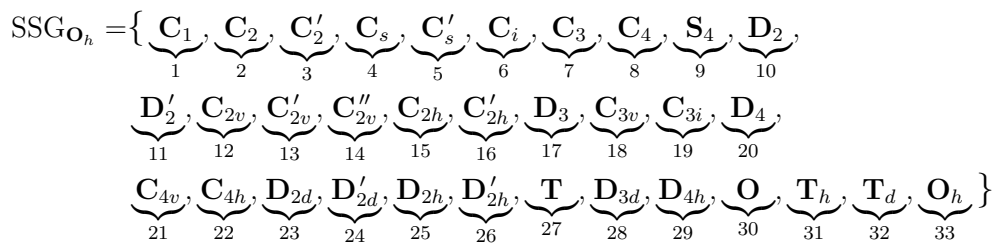

where the subgroups are aligned and numbered sequentially in the ascending order of their orders.

The author (Fujita) has calculated the following standard mark table of the point group $\mathbf{O}_{h}$ by means of the FORTRAN77 calculation.

Standard Mark Table for the Point Group $\mathbf{O}_{h}$ (Oh_cube)

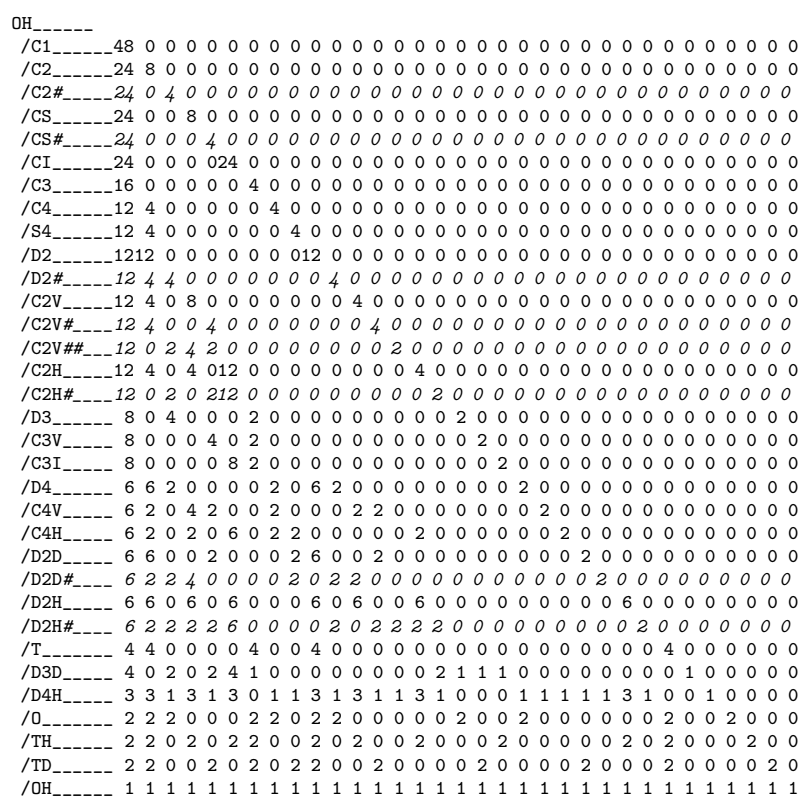

The mark table generated by the GAP function Table0fMarks is sorted to be converted into the standard mark table (obeying $\mathrm{SSG}_{\mathbf{O}_{h}}$ of Eq. 7) [30]. This calculation depending on the GAP system by using CPRs is consistent with the results due to the FORTRAN77 calculation [20,21].

The resulting standard mark table of $\mathbf{O}_{h}$ is applied to calculate partial-cycle indices 
with chirality fittingness (PCI-CFs) [20] as shown below.

PCI-CFs under the point group $\mathbf{O}_{h}$

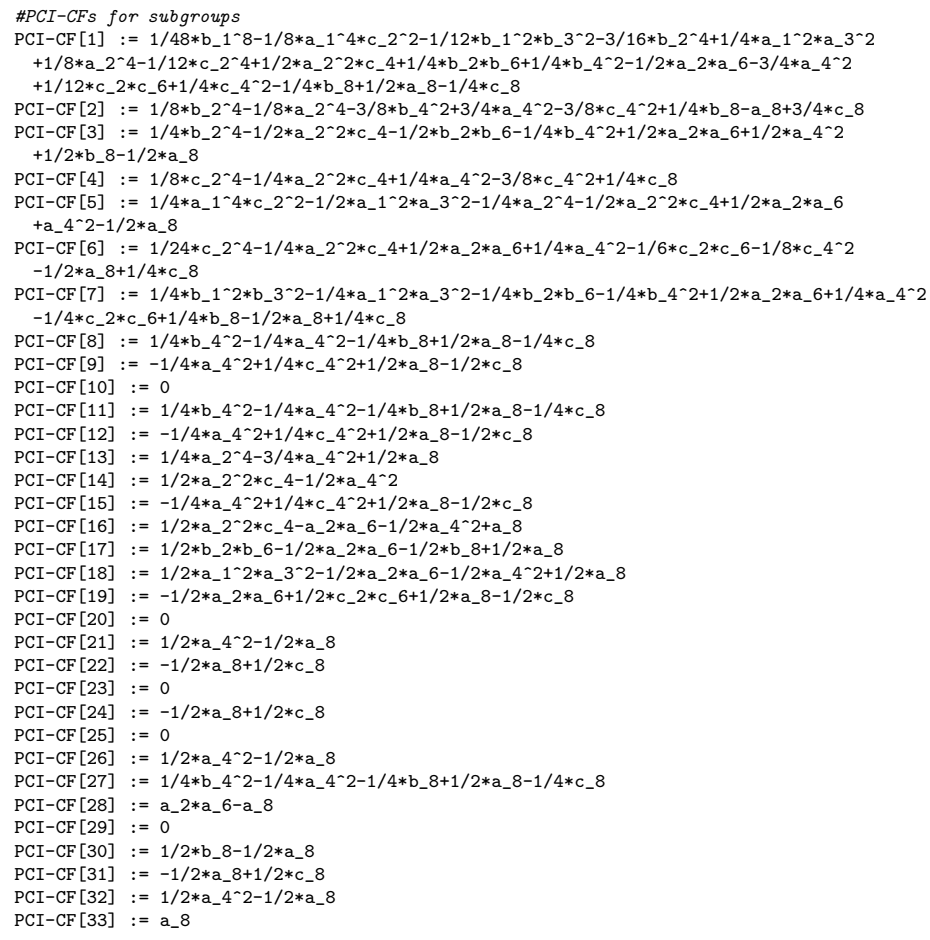

These PCI-CFs in the GAP expressions are converted into formulas of usual mathe- 
matic conventions as follows:

$$
\begin{aligned}
& \mathrm{PCI}-\mathrm{CF}_{\mathbf{O}_{h} \mathbf{1}}\left(\mathbf{C}_{1}, \$_{d}\right)=\frac{1}{48} b_{1}^{8}-\frac{1}{8} c_{2}^{2} a_{1}^{4}-\frac{1}{12} b_{1}^{2} b_{3}^{2}-\frac{3}{16} b_{2}^{4}-\frac{1}{12} c_{2}^{4}+\frac{1}{4} a_{1}^{2} a_{3}^{2} \\
& +\frac{1}{8} a_{2}^{4}+\frac{1}{2} c_{4} a_{2}^{2}+\frac{1}{4} b_{2} b_{6}+\frac{1}{12} c_{2} c_{6}+\frac{1}{4} b_{4}^{2}+\frac{1}{4} c_{4}^{2}-\frac{1}{2} a_{2} a_{6}-\frac{3}{4} a_{4}^{2} \\
& -\frac{1}{4} b_{8}-\frac{1}{4} c_{8}+\frac{1}{2} a_{8} \\
& \text { PCI-CF } \mathbf{O}_{h} \mathbf{1}\left(\mathbf{C}_{2}, \$_{d}\right)=\frac{1}{8} b_{2}^{4}-\frac{1}{8} a_{2}^{4}-\frac{3}{8} b_{4}^{2}-\frac{3}{8} c_{4}^{2}+\frac{3}{4} a_{4}^{2}+\frac{1}{4} b_{8}+\frac{3}{4} c_{8}-a_{8} \\
& \text { PCI-CF } \mathbf{O}_{h} \mathbf{1}\left(\mathbf{C}_{2}^{\prime}, \$_{d}\right)=\frac{1}{4} b_{2}^{4}-\frac{1}{2} c_{4} a_{2}^{2}-\frac{1}{2} b_{2} b_{6}-\frac{1}{4} b_{4}^{2}+\frac{1}{2} a_{2} a_{6}+\frac{1}{2} a_{4}^{2}+\frac{1}{2} b_{8}-\frac{1}{2} a_{8} \\
& \text { PCI-CF } \mathbf{O}_{h} \mathbf{1}\left(\mathbf{C}_{s}, \$_{d}\right)=\frac{1}{8} c_{2}^{4}-\frac{1}{4} c_{4} a_{2}^{2}-\frac{3}{8} c_{4}^{2}+\frac{1}{4} a_{4}^{2}+\frac{1}{4} c_{8} \\
& \text { PCI-CF } \mathbf{O}_{h} \mathbf{1}\left(\mathbf{C}_{s}^{\prime}, \$_{d}\right)=\frac{1}{4} c_{2}^{2} a_{1}^{4}-\frac{1}{2} a_{1}^{2} a_{3}^{2}-\frac{1}{4} a_{2}^{4}-\frac{1}{2} c_{4} a_{2}^{2}+\frac{1}{2} a_{2} a_{6}+a_{4}^{2}-\frac{1}{2} a_{8} \\
& \text { PCI-CF } \mathbf{O}_{h} \mathbf{1}\left(\mathbf{C}_{i}, \$_{d}\right)=\frac{1}{24} c_{2}^{4}-\frac{1}{4} c_{4} a_{2}^{2}-\frac{1}{6} c_{2} c_{6}-\frac{1}{8} c_{4}^{2}+\frac{1}{2} a_{2} a_{6}+\frac{1}{4} a_{4}^{2} \\
& +\frac{1}{4} c_{8}-\frac{1}{2} a_{8} \\
& \text { PCI-CF } \mathbf{O}_{h} \mathbf{1}\left(\mathbf{C}_{3}, \$_{d}\right)=\frac{1}{4} b_{1}^{2} b_{3}^{2}-\frac{1}{4} a_{1}^{2} a_{3}^{2}-\frac{1}{4} b_{2} b_{6}-\frac{1}{4} c_{2} c_{6}-\frac{1}{4} b_{4}^{2}+\frac{1}{2} a_{2} a_{6}+\frac{1}{4} a_{4}^{2} \\
& +\frac{1}{4} b_{8}+\frac{1}{4} c_{8}-\frac{1}{2} a_{8} \\
& \text { PCI-CF } \mathbf{O}_{h} \mathbf{1}\left(\mathbf{C}_{4}, \$_{d}\right)=\frac{1}{4} b_{4}^{2}-\frac{1}{4} a_{4}^{2}-\frac{1}{4} b_{8}-\frac{1}{4} c_{8}+\frac{1}{2} a_{8} \\
& \text { PCI-CF } \mathbf{O}_{h} \mathbf{1}\left(\mathbf{S}_{4}, \$_{d}\right)=\frac{1}{4} c_{4}^{2}-\frac{1}{4} a_{4}^{2}-\frac{1}{2} c_{8}+\frac{1}{2} a_{8} \\
& \text { PCI-CF } \mathbf{O}_{h} \mathbf{1}\left(\mathbf{D}_{2}, \$_{d}\right)=0 \\
& \text { PCI-CF } \mathbf{O}_{h} \mathbf{1}\left(\mathbf{D}_{2}^{\prime}, \$_{d}\right)=\frac{1}{4} b_{4}^{2}-\frac{1}{4} a_{4}^{2}-\frac{1}{4} b_{8}-\frac{1}{4} c_{8}+\frac{1}{2} a_{8} \\
& \text { PCI-CF } \mathbf{O}_{h} \mathbf{1}\left(\mathbf{C}_{2 v}, \$_{d}\right)=\frac{1}{4} c_{4}^{2}-\frac{1}{4} a_{4}^{2}-\frac{1}{2} c_{8}+\frac{1}{2} a_{8} \\
& \text { PCI-CF } \mathbf{O}_{h} \mathbf{1}\left(\mathbf{C}_{2 v}^{\prime}, \$_{d}\right)=\frac{1}{4} a_{2}^{4}-\frac{3}{4} a_{4}^{2}+\frac{1}{2} a_{8} \\
& \text { PCI-CF } \mathbf{O}_{h} \mathbf{1}\left(\mathbf{C}_{2 v}^{\prime \prime}, \$_{d}\right)=\frac{1}{2} c_{4} a_{2}^{2}-\frac{1}{2} a_{4}^{2} \\
& \text { PCI-CF } \mathbf{O}_{h} \mathbf{1}\left(\mathbf{C}_{2 h}, \$_{d}\right)=\frac{1}{4} c_{4}^{2}-\frac{1}{4} a_{4}^{2}-\frac{1}{2} c_{8}+\frac{1}{2} a_{8} \\
& \text { PCI-CF } \mathbf{O}_{h} \mathbf{1}\left(\mathbf{C}_{2 h}^{\prime}, \$_{d}\right)=\frac{1}{2} c_{4} a_{2}^{2}-a_{2} a_{6}-\frac{1}{2} a_{4}^{2}+a_{8} \\
& \text { PCI-CF } \mathbf{O}_{h} \mathbf{1}\left(\mathbf{D}_{3}, \$_{d}\right)=\frac{1}{2} b_{2} b_{6}-\frac{1}{2} a_{2} a_{6}-\frac{1}{2} b_{8}+\frac{1}{2} a_{8}
\end{aligned}
$$




$$
\begin{aligned}
& \text { PCI-CF } \mathbf{O}_{h} \mathbf{1}\left(\mathbf{C}_{3 v}, \$_{d}\right)=\frac{1}{2} a_{1}^{2} a_{3}^{2}-\frac{1}{2} a_{2} a_{6}-\frac{1}{2} a_{4}^{2}+\frac{1}{2} a_{8} \\
& \text { PCI-CF } \mathbf{O}_{h} \mathbf{1}\left(\mathbf{C}_{3 i}, \$_{d}\right)=\frac{1}{2} c_{2} c_{6}-\frac{1}{2} a_{2} a_{6}-\frac{1}{2} c_{8}+\frac{1}{2} a_{8} \\
& \text { PCI-CF } \mathbf{O}_{h} \mathbf{1}\left(\mathbf{D}_{4}, \$_{d}\right)=0 \\
& \text { PCI-CF } \mathbf{O}_{h} \mathbf{1}\left(\mathbf{C}_{4 v}, \$_{d}\right)=\frac{1}{2} a_{4}^{2}-\frac{1}{2} a_{8} \\
& \text { PCI-CF } \mathbf{O}_{h} \mathbf{1}\left(\mathbf{C}_{4 h}, \$_{d}\right)=\frac{1}{2} c_{8}-\frac{1}{2} a_{8} \\
& \text { PCI-CF } \mathbf{O}_{h} \mathbf{1}\left(\mathbf{D}_{2 d}, \$_{d}\right)=0 \\
& \text { PCI-CF } \mathbf{O}_{h} \mathbf{1}\left(\mathbf{D}_{2 d}^{\prime}, \$_{d}\right)=\frac{1}{2} c_{8}-\frac{1}{2} a_{8} \\
& \text { PCI-CF } \mathbf{O}_{h} \mathbf{1}\left(\mathbf{D}_{2 h}, \$_{d}\right)=0 \\
& \text { PCI-CF } \mathbf{O}_{h} \mathbf{1}\left(\mathbf{D}_{2 h}^{\prime}, \$_{d}\right)=\frac{1}{2} a_{4}^{2}-\frac{1}{2} a_{8} \\
& \text { PCI-CF } \mathbf{O}_{h} \mathbf{1}\left(\mathbf{T}, \$_{d}\right)=\frac{1}{4} b_{4}^{2}-\frac{1}{4} a_{4}^{2}-\frac{1}{4} b_{8}-\frac{1}{4} c_{8}+\frac{1}{2} a_{8} \\
& \text { PCI-CF } \mathbf{O}_{h} \mathbf{1}\left(\mathbf{D}_{3 d}, \$_{d}\right)=a_{2} a_{6}-a_{8} \\
& \text { PCI-CF } \mathbf{O}_{h} \mathbf{1}\left(\mathbf{D}_{4 h}, \$_{d}\right)=0 \\
& \text { PCI-CF } \text { O }_{h} \mathbf{1}\left(\mathbf{O}, \$_{d}\right)=\frac{1}{2} b_{8}-\frac{1}{2} a_{8} \\
& \text { PCI-CF } \mathbf{O}_{h} \mathbf{1}\left(\mathbf{T}_{h}, \$_{d}\right)=\frac{1}{2} c_{8}-\frac{1}{2} a_{8} \\
& \text { PCI-CF } \mathbf{O}_{h} \mathbf{1}\left(\mathbf{T}_{d}, \$_{d}\right)=\frac{1}{2} a_{4}^{2}-1 / 2 a_{8} \\
& \text { PCI-CF } \mathbf{O}_{h} \mathbf{1}\left(\mathbf{O}_{h}, \$_{d}\right)=a_{8}
\end{aligned}
$$

The results of Eqs. 8-40 are consistent with Eqs. 6-38 of Ref. [20], where the 7th term $-\frac{1}{8} a_{4}^{2}$ in Eq. 7 of Ref. [20] (corresponding to Eq. 9 of present article) should be read as $-\frac{1}{8} a_{2}^{4}$

\subsection{Partial cycle indices with chirality fittingness (PCI-CFs) un- der the $R S$-stereogenic group $\mathrm{O}_{\widetilde{\sigma}}$}

The $R S$-stereogenic group $\mathbf{O}_{\widetilde{\sigma}}$ (order 48) has 33 subgroups up to conjugacy, which can be aligned in the corresponding sequence to $\operatorname{SSG}_{\mathbf{O}_{h}}$ (Eq. 7). 


$$
\begin{aligned}
\operatorname{SSG}_{\mathbf{O}_{\tilde{\sigma}}}= & \{\underbrace{\mathbf{C}_{1}}_{1}, \underbrace{\mathbf{C}_{2}}_{2}, \underbrace{\mathbf{C}_{2}^{\prime}}_{3}, \underbrace{\widetilde{\mathbf{C}}_{s}}_{4}, \underbrace{\widetilde{\mathbf{C}}_{s}^{\prime}}_{5}, \underbrace{\widetilde{\mathbf{C}}_{i}}_{6}, \underbrace{\mathbf{C}_{3}}_{7}, \underbrace{\mathbf{C}_{4}}_{8}, \underbrace{\widetilde{\mathbf{S}}_{4}}_{9}, \underbrace{\mathbf{D}_{2}}_{10}, \\
& \underbrace{\mathbf{D}_{2}^{\prime}}_{11}, \underbrace{\widetilde{\mathbf{C}}_{2 v}}_{12}, \underbrace{\widetilde{\mathbf{C}}_{2 v}^{\prime}}_{13}, \underbrace{\widetilde{\mathbf{C}}_{2 v}^{\prime \prime}}_{14}, \underbrace{\widetilde{\mathbf{C}}_{2 h}}_{15}, \underbrace{\widetilde{\mathbf{C}}_{2 h}^{\prime}}_{16}, \underbrace{\mathbf{D}_{3}}_{17}, \underbrace{\widetilde{\mathbf{C}}_{3 v}}_{18}, \underbrace{\widetilde{\mathbf{C}}_{3 i}}_{19}, \underbrace{\mathbf{D}_{4}}_{20}, \\
& \underbrace{\widetilde{\mathbf{C}}_{4 v}}_{21}, \underbrace{\widetilde{\mathbf{C}}_{4 h}}_{22}, \underbrace{\widetilde{\mathbf{D}}_{2 d}}_{23}, \underbrace{\widetilde{\mathbf{D}}_{2 d}^{\prime}}_{24}, \underbrace{\widetilde{\mathbf{D}}_{2 h}}_{25}, \underbrace{\widetilde{\mathbf{D}}_{2 h}^{\prime}}_{26}, \underbrace{\mathbf{T}}_{27}, \underbrace{\widetilde{\mathbf{D}}_{3 d}}_{28}, \underbrace{\widetilde{\mathbf{D}}_{4 h}}_{29}, \underbrace{\mathbf{O}}_{30}, \underbrace{\widetilde{\mathbf{T}}_{h}}_{31}, \underbrace{\widetilde{\mathbf{T}}_{d}}_{32}, \underbrace{\mathbf{O}_{\tilde{\sigma}}}_{33}\}
\end{aligned}
$$

where the subgroups are aligned and numbered sequentially in the ascending order of their orders. As found easily, $\mathrm{SSG}_{\mathbf{O}_{h}}$ (Eq. 7) and $\mathrm{SSG}_{\mathbf{O}_{\tilde{\sigma}}}$ (Eq. 41) commonly contain the rotations due to the chiral subgroup $\mathbf{O}$. Each subgroup with a tilde accent in $\operatorname{SSG}_{\mathbf{O}_{\tilde{\sigma}}}$ (Eq. 41 ) is generated from the corresponding subgroup without a tilde accent in $\mathrm{SSG}_{\mathbf{O}_{h}}$ (Eq. 7), where a 2-cycle (9 10) (or an overline) of each permutation for reflection is detached to give a permutation with no reflection. For example, the subgroup $\widetilde{\mathbf{C}}_{s}$ in Eq. 41 (with a set of generators gen [4] := $[(1,5)(2,6)(3,7)(4,8)]$; ; ) is generated by omitting a 2-cycle $\left(\begin{array}{ll}9 & 10\end{array}\right)$ from the subgroup $\mathbf{C}_{s}$ in Eq. 7 (with a set of generators: gen [4] $:=[(1,5)(2,6)(3,7)(4,8)(9,10)] ; ;)$.

The mark table generated by the GAP function Table0fMarks is sorted in a parallel way to be converted into the mark table obeying $\operatorname{SSG}_{\mathbf{O}_{\tilde{\sigma}}}$ (Eq. 41). The apparent feature of the resulting mark table of $\mathbf{O}_{\widetilde{\sigma}}$ is identical with the standard mark table of $\mathbf{O}_{h}$ under the GAP system.

On the basis of the mark table obeying $\operatorname{SSG}_{\mathbf{O}_{\tilde{\sigma}}}$ (Eq. 41), a set of partial-cycle indices with chirality fittingness (PCI-CFs) is calculated as follows:

PCI-CFs under the the $R S$-stereogenic group $\mathbf{O}_{\tilde{\sigma}}$

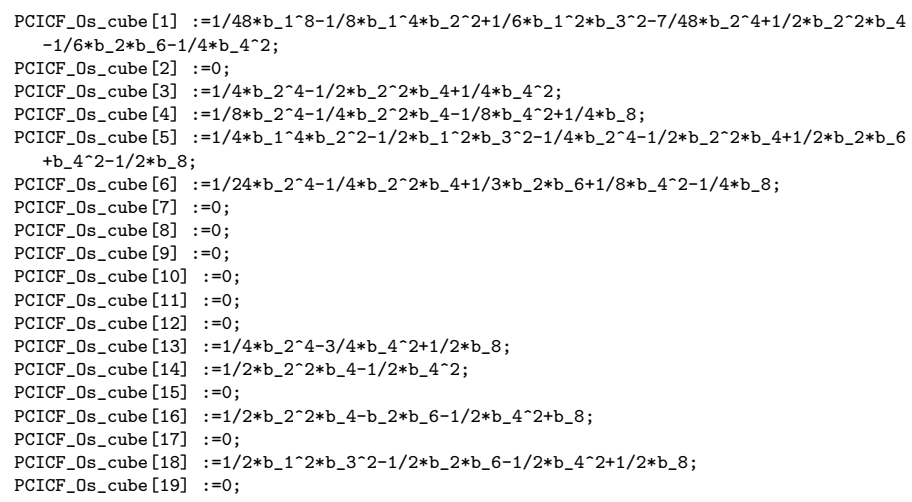


PCICF_Os_cube [20] : : 0 ;

PCICF_Os_cube [21] ::=1/2*b_4^2-1/2*b_8;

PCICF_Os_cube [22] : : 0 ;

PCICF_Os_cube [23] : $=0$;

PCICF_Os_cube [24] : :=0;

PCICF_Os_cube [25] : : =0;

PCICF_Os_cube [26] ::=1/2*b_4^²-1/2*b_8;

PCICF_0s_cube [27] : $=0$;

PCICF_0s_cube [28] :=b_2*b_6-b_8;

PCICF_0s_cube [29] : =0;

PCICF_0s_cube [30] : $=0$;

PCICF_0s_cube [31] : :=0;

PCICF_Os_cube [32] : $=1 / 2 * b_{-} 4{ }^{\wedge} 2-1 / 2 * b_{-} 8$;

PCICF_Os_cube [33] :=b_8;

It should be noted that sphericity indices due to $\$_{d}\left(a_{d}, b_{d}, c_{d}\right)$ are degenerated into a single sphericity index $b_{d}$. The resulting PCI-CFs contain a single kind of chirality fittingness due to $b_{d}$, so that many PCI-CFs (19 among 33 PCI-CFs) vanish into zero, as found the above codes. These PCI-CFs in the GAP expressions are represented by usual mathematic conventions as follows:

$$
\begin{aligned}
& \mathrm{PCI}-\mathrm{CF}_{\mathbf{O}_{\tilde{\sigma}} \mathbf{1}}\left(\mathbf{C}_{1}, b_{d}\right)=\frac{1}{48} b_{1}^{8}-\frac{1}{8} b_{1}^{4} b_{2}^{2}+\frac{1}{6} b_{1}^{2} b_{3}^{2} \\
& -\frac{7}{48} b_{2}^{4}+\frac{1}{2} b_{2}^{2} b_{4}-\frac{1}{6} b_{2} b_{6}-\frac{1}{4} b_{4}^{2} \\
& \text { PCI-CF } \mathbf{O}_{\tilde{\sigma}} \mathbf{1}\left(\mathbf{C}_{2}, b_{d}\right)=0 \\
& \text { PCI-CF } \mathbf{O}_{\tilde{\sigma} \mathbf{1}}\left(\mathbf{C}_{2}^{\prime}, b_{d}\right)=\frac{1}{4} b_{2}^{4}-\frac{1}{2} b_{2}^{2} b_{4}+\frac{1}{4} b_{4}^{2} \\
& \text { PCI-CF } \mathbf{O}_{\tilde{\sigma}} \mathbf{1}\left(\widetilde{\mathbf{C}}_{s}, b_{d}\right)=\frac{1}{8} b_{2}^{4}-\frac{1}{4} b_{2}^{2} b_{4}-\frac{1}{8} b_{4}^{2}+\frac{1}{4} b_{8} \\
& \text { PCI-CF }{\mathbf{\mathbf { O } _ { \tilde { \sigma } } \mathbf { 1 }}}_{\mathbf{1}}\left(\widetilde{\mathbf{C}}_{s}^{\prime}, b_{d}\right)=\frac{1}{4} b_{1}^{4} b_{2}^{2}-\frac{1}{2} b_{1}^{2} b_{3}^{2}-\frac{1}{4} b_{2}^{4}-\frac{1}{2} b_{2}^{2} b_{4} \\
& +\frac{1}{2} b_{2} b_{6}+b_{4}^{2}-\frac{1}{2} b_{8} \\
& \text { PCI-CF } \mathbf{O}_{\tilde{\sigma}} \mathbf{1}\left(\widetilde{\mathbf{C}}_{i}, b_{d}\right)=\frac{1}{24} b_{2}^{4}-\frac{1}{4} b_{2}^{2} b_{4}+\frac{1}{3} b_{2} b_{6}+\frac{1}{8} b_{4}^{2}-\frac{1}{4} b_{8} \\
& \text { PCI-CF } \mathbf{O}_{\tilde{\sigma} \mathbf{1}}\left(\mathbf{C}_{3}, b_{d}\right)=0 \\
& \text { PCI-CF } \mathbf{O}_{\tilde{\sigma} \mathbf{1}}\left(\mathbf{C}_{4}, b_{d}\right)=0 \\
& \text { PCI-CF } \mathbf{O}_{\tilde{\sigma} \mathbf{1}}\left(\widetilde{\mathbf{S}}_{4}, b_{d}\right)=0 \\
& \text { PCI-CF }{\text { O } \mathbf{O}_{\tilde{\sigma}} \mathbf{1}}\left(\mathbf{D}_{2}, b_{d}\right)=0 \\
& \mathrm{PCI}-\mathrm{CF}_{\mathbf{O}_{\tilde{\sigma}} \mathbf{1}}\left(\mathbf{D}_{2}^{\prime}, b_{d}\right)=0 \\
& \text { PCI-CF } \mathbf{O}_{\tilde{\sigma} \mathbf{1}}\left(\widetilde{\mathbf{C}}_{2 v}, b_{d}\right)=0 \\
& \text { PCI-CF }{\mathbf{\mathbf { O } _ { \tilde { \sigma } } \mathbf { 1 }}}\left(\widetilde{\mathbf{C}}_{2 v}^{\prime}, b_{d}\right)=\frac{1}{4} b_{2}^{4}-\frac{3}{4} b_{4}^{2}+\frac{1}{2} b_{8} ; \\
& \text { PCI-CF } \mathbf{O}_{\tilde{\sigma} \mathbf{1}}\left(\widetilde{\mathbf{C}}_{2 v}^{\prime \prime}, b_{d}\right)=\frac{1}{2} b_{2}^{2} b_{4}-\frac{1}{2} b_{4}^{2} ;
\end{aligned}
$$




$$
\begin{aligned}
& \text { PCI-CF } \mathbf{O}_{\tilde{\sigma} \mathbf{1}}\left(\widetilde{\mathbf{C}}_{2 h}, b_{d}\right)=0 \\
& \text { PCI-CF } \mathbf{O}_{\tilde{\sigma}} \mathbf{1}\left(\widetilde{\mathbf{C}}_{2 h}^{\prime}, b_{d}\right)=\frac{1}{2} b_{2}^{2} b_{4}-b_{2} b_{6}-\frac{1}{2} b_{4}^{2}+b_{8} ; \\
& \text { PCI-CF } \mathbf{O}_{\tilde{\sigma} \mathbf{1}}\left(\mathbf{D}_{3}, b_{d}\right)=0 \\
& \text { PCI-CF } \mathbf{O}_{\tilde{\sigma}} \mathbf{1}\left(\widetilde{\mathbf{C}}_{3 v}, b_{d}\right)=\frac{1}{2} b_{1}^{2} b_{3}^{2}-\frac{1}{2} b_{2} b_{6}-\frac{1}{2} b_{4}^{2}+\frac{1}{2} b_{8} ; \\
& \text { PCI-CF } \mathbf{O}_{\tilde{\sigma}} \mathbf{1}\left(\widetilde{\mathbf{C}}_{3 i}, b_{d}\right)=0
\end{aligned}
$$

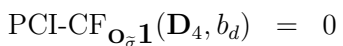

$$
\begin{aligned}
& \text { PCI-CF } \mathbf{O}_{\widetilde{\sigma}} \mathbf{1}\left(\widetilde{\mathbf{C}}_{4 v}, b_{d}\right)=\frac{1}{2} b_{4}^{2}-\frac{1}{2} b_{8} ; \\
& \text { PCI-CF } \mathbf{O}_{\tilde{\sigma} \mathbf{1}}\left(\widetilde{\mathbf{C}}_{4 h}, b_{d}\right)=0 \\
& \text { PCI-CF } \mathbf{O}_{\tilde{\sigma} \mathbf{1}}\left(\widetilde{\mathbf{D}}_{2 d}, b_{d}\right)=0 \\
& \text { PCI-CF } \mathbf{O}_{\tilde{\sigma} \mathbf{1}}\left(\widetilde{\mathbf{D}}_{2 d}^{\prime}, b_{d}\right)=0 \\
& \text { PCI-CF } \mathbf{O}_{\tilde{\sigma} \mathbf{1}}\left(\widetilde{\mathbf{D}}_{2 h}, b_{d}\right)=0 \\
& \text { PCI-CF }{\mathbf{\mathbf { O } _ { \tilde { \sigma } }}}_{\mathbf{1}}\left(\widetilde{\mathbf{D}}_{2 h}^{\prime}, b_{d}\right)=\frac{1}{2} b_{4}^{2}-\frac{1}{2} b_{8} \\
& \text { PCI-CF } \mathbf{O}_{\tilde{\sigma}} \mathbf{1}\left(\mathbf{T}, b_{d}\right)=0 \\
& \text { PCI-CF } \mathbf{O}_{\widetilde{\sigma}} \mathbf{1}\left(\widetilde{\mathbf{D}}_{2 h}, b_{d}\right)=b_{2} b_{6}-b_{8} \\
& \text { PCI-CF }{ }_{\mathbf{O}_{\tilde{\sigma}} \mathbf{1}}\left(\widetilde{\mathbf{D}}_{4 h}, b_{d}\right)=0 \\
& \text { PCI-CF } \mathbf{O}_{\tilde{\sigma}} \mathbf{1}\left(\mathbf{O}, b_{d}\right)=0 \\
& \text { PCI-CF } \mathbf{O}_{\tilde{\sigma} \mathbf{1}}\left(\widetilde{\mathbf{T}}_{h}, b_{d}\right)=0 \\
& \text { PCI-CF } \mathbf{O}_{\tilde{\sigma}} \mathbf{1}\left(\widetilde{\mathbf{T}}_{d}, b_{d}\right)=\frac{1}{2} b_{4}^{2}-\frac{1}{2} b_{8} ; \\
& \text { PCI-CF } \mathbf{O}_{\tilde{\sigma} \mathbf{1}}\left(\mathbf{O}_{\tilde{\sigma}}, b_{d}\right)=b_{8}
\end{aligned}
$$

\subsection{Partial cycle indices with chirality fittingness (PCI-CFs) un- der the $L R$-permutation group $\mathrm{O}_{\widehat{I}}$}

The $L R$-permutation group $\mathbf{O}_{\widehat{I}}$ (OI_cube) is obtained by using the set of generators gen_4, which is generated by adding the 2-cycle $(9,10)$ to the set of generators gen_1 for the chiral subgroup $\mathbf{O}$. In order to calculate PCIs under $L R$-permutation group $\mathbf{O}_{\widehat{I}}$ (OI_cube), the corresponding mark table (table of marks) named tom_OI_cube is first obtained by using the GAP function Table0fMarks as follows:

gap> OI_cube := $\operatorname{Group}([(1,2,3,4)(5,6,7,8),(2,4,5)(3,8,6),(9,10)])$; ; 
gap> tom_OI_cube := TableOfMarks (OI_cube);

TableOfMarks ( $\operatorname{Group}([(1,2,3,4)(5,6,7,8),(2,4,5)(3,8,6),(9,10)]))$

gap> Display (tom_OI_cube);

The produced mark table tom_OI_cube obeys the format of the GAP system, where the alignment of the subgroups is in accord with the routine of the GAP system. No standardization is attempted (cf. Ref. [30]), because the $L R$-permutation group $\mathbf{O}_{\widehat{I}}$ (OI_cube) does not correspond to the point group $\mathbf{O}_{h}$ of the cubane skeleton. Hence, the following mark table tom_OI_cube obtained by the GAP function Table0fMarks is regarded as a standard mark table as it is:

Mark table (tom_OI_cube) for the $L R$-permutation group $\mathbf{O}_{\widehat{I}}$ (OI_cube)

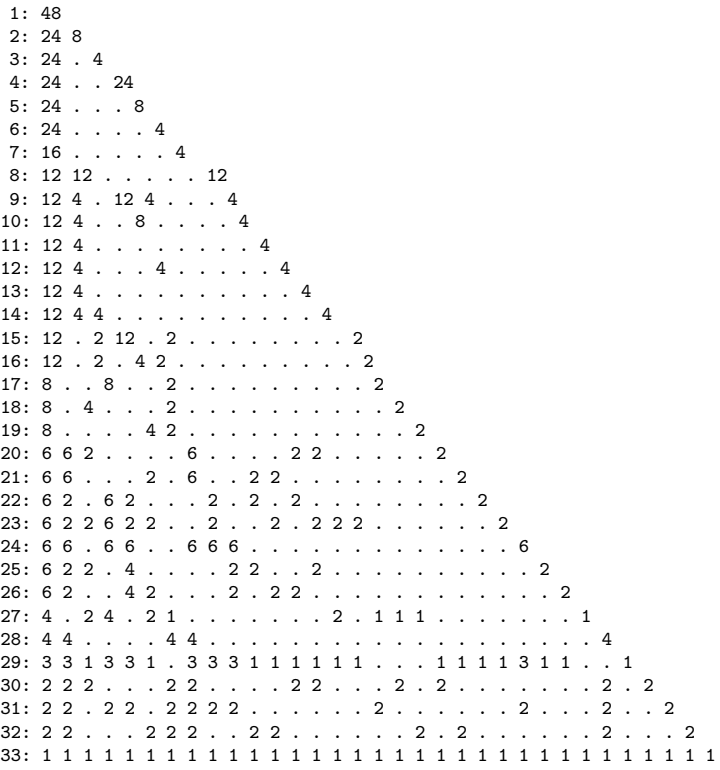

Each row (and each column) of the mark table (tom_OI_cube) is in accord with the SSG of the $L R$-permutation group $\mathrm{SSG}_{\mathbf{O}_{\widehat{I}}}$. The sequence of the $\mathrm{SSG}$ is calculated by means of the GAP function RepresentativeTom and the respective set of generators (gen [i] for $i=1 \cdots 33)$ is calculated by the GAP function Generators0fGroup:

gap> gen $:=[] ;$;

gap> for $i$ in [1..33] do

$>$ gen [i] := RepresentativeTom (tom_OI_cube, $i$ );

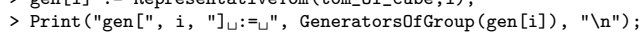

$>$ od; 
Thereby, the sets of generators for producing the respective rows of subgroups of the mark table tom_OI_cube are obtained:

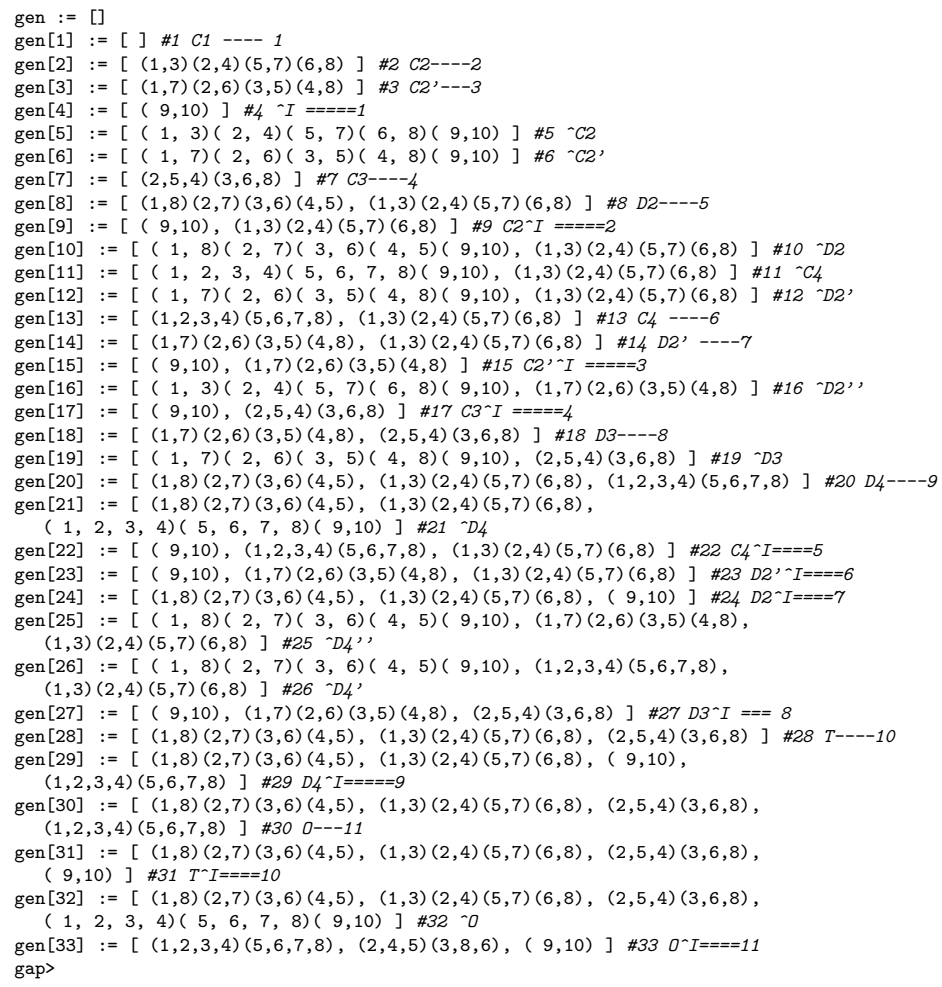

The assigned subgroup is added after the \# symbol in the right-side end of each set of generators. These data can be summarized into the following set of subgroups $\mathrm{SSG}_{\mathbf{O}_{\widehat{I}}}$.

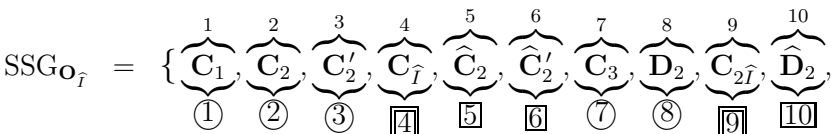

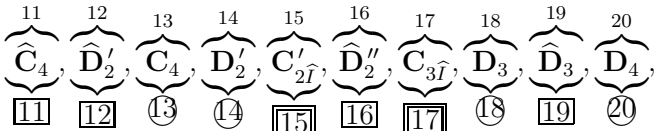

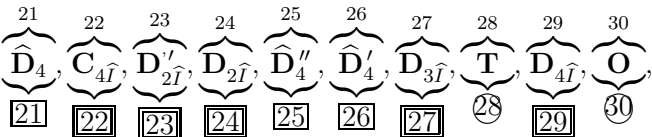




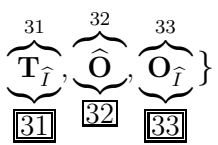

This set of subgroups $\mathrm{SSG}_{\mathbf{O}_{\widehat{I}}}$ contains eleven subgroups coming from the maximum chiral subgroup $\mathbf{O}$, the sequential numbers of which are encircled by a circle(e.g., (1), (2), ‥ (30) (see Eq. 1). On the other hand, this set of subgroups $\mathrm{SSG}_{\mathbf{O}_{\widehat{I}}}$ contains eleven subgroups containing an $L R$-permutation $\widehat{I}$ represented by an independent 2-cycle symbol (9 10), the sequential numbers of which are surrounded by a double frame box

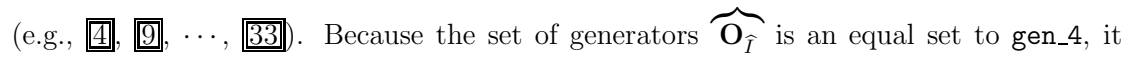
33

generates the $L R$-permutation group $\mathbf{O}_{\widehat{I}}$ (OI_cube). This fact is testified by the following GAP functions. The coset decomposition due to the GAP function CosetDecomposition (CD_OI_O) is described above.

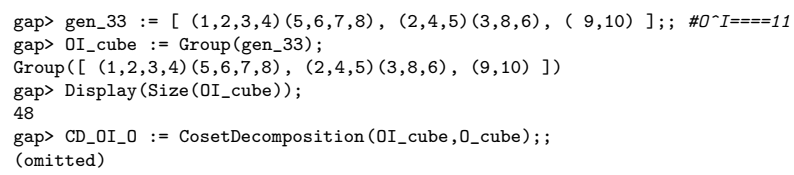

The resulting $L R$-permutation group $\mathbf{O}_{\widehat{I}}$ (OI_cube) (order 48) contains the subgroup $\mathbf{T}_{\widehat{I}}$ (TI_cube) (order 24), which is generated by means of the set of generators gen_31. Their supergroup-subgroup relationship is confirmed by the GAP function IsSubgroup.

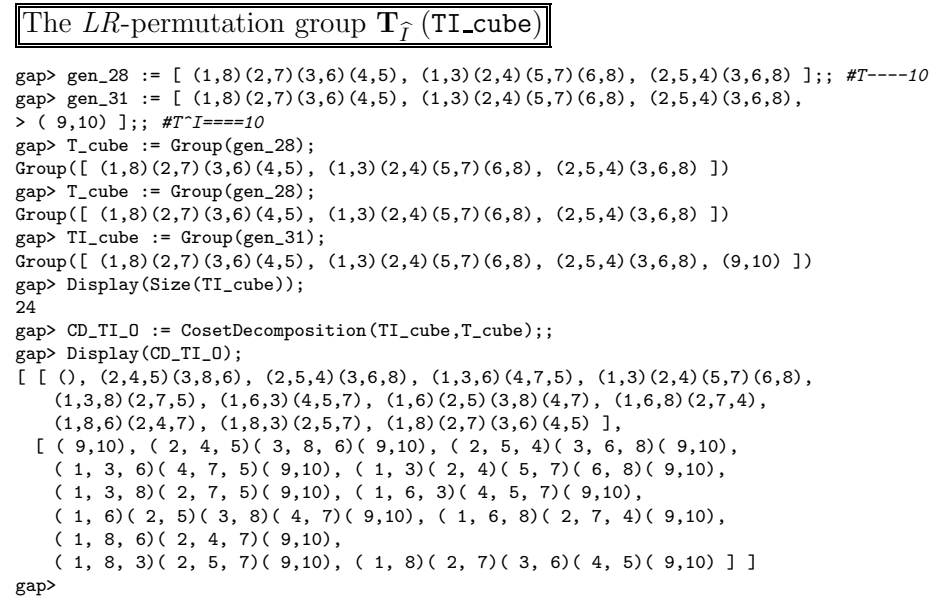


The set of generators gen_30 generates the point group $\mathbf{O}$, which is also generated by means of the set of generators gen_1

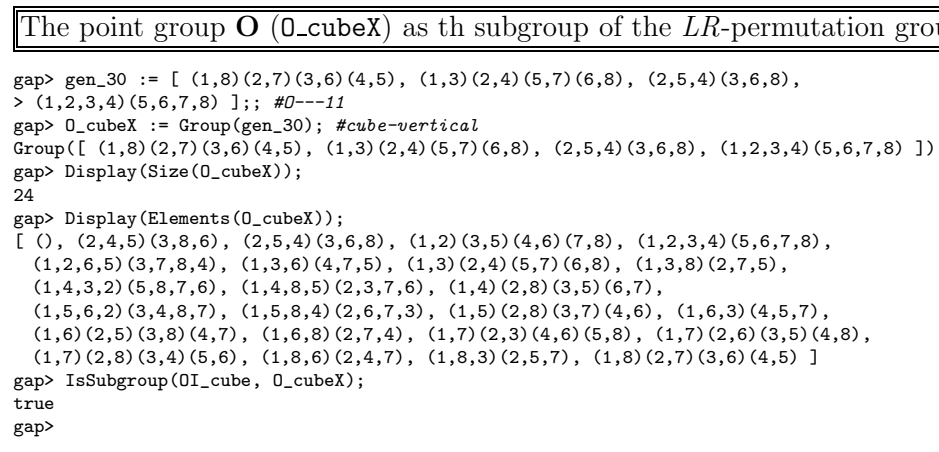

Compare 0_cube (from gen_1) and 0_cubeX (from gen_30). Although their sets of generators gen_1 and gen_30 are not equal to each other, the resulting groups 0_cube and O_cubeX contain the same set of elements, as confirmed by the following GAP code:

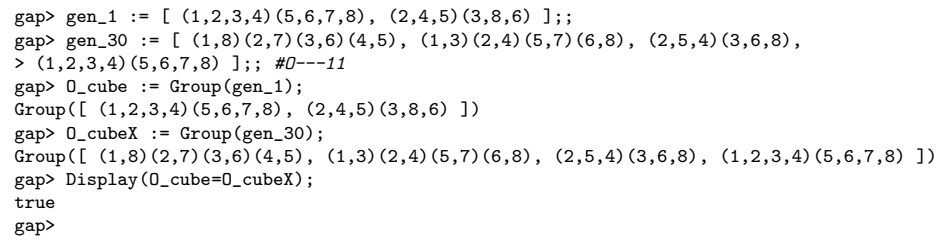

Finally, the remaining eleven subgroups are designated by the sequential numbers of which are surrounded by a single frame box (e.g., 5, 10, ․ 32).

As listed in Appendix B, the GAP functions developed for the concordant generation of mark tables and USCI-CF tables can be applied to this unsorted case by using the SSG shown as $\mathrm{SSG}_{\mathbf{O}_{\widehat{I}}}$ (Eq. 75).

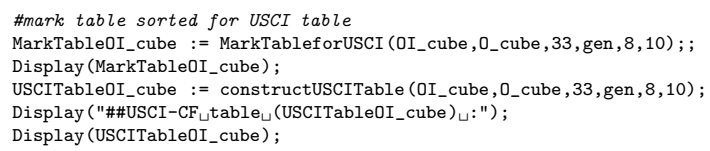

The resulting standard mark table MarkTableOI_cube is identical with the unsorted mark table (tom_OI_cube cited above) in this case of the $L R$-permutation group $\mathbf{O}_{\widehat{I}}$ (OI_cube). The concordantly generated USCI table USCITableOI_cube is obtained as a list having 33 inner lists $[[\cdots] \cdots[\cdots] \cdots[\cdots]]$, each of which is surrounded by an inner pair of square brackets. For the simplicity's sake, the 17th inner list is extracted in the 
following output.

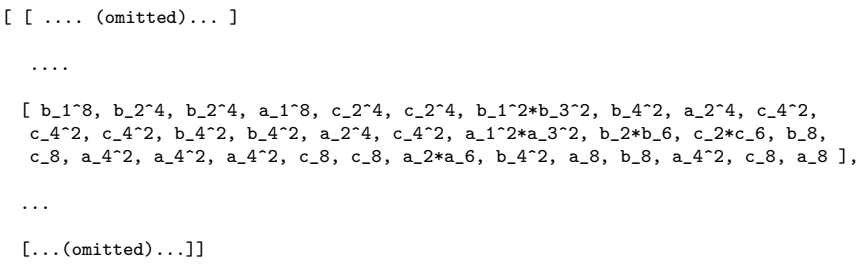

To accomplish further calculations, the standard mark table MarkTableOI_cube is converted into a matrix format by using GAP function MatTom as follows. The resulting matrix named Matrix_tomOI_cube is a GAP list format, in which an outer list contains 33 rows of inner lists.

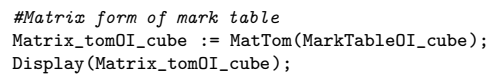

The newly-developed GAP function calculateFPvector gives a fixed-point vector FPVcube for characterizing eight substitution positions of a holantimeric cubane skeleton $\overline{2}(\mathrm{D})$. 
\#Fixed point vector for cube

$[8,0,0,8,0,0,2,0,0,0,0,0,0,0,0,0,2,0,0,0$,

$0,0,0,0,0,0,0,0,0,0,0,0,0]$

Because the eight positions of $\overline{\mathbf{2}}$ belong to one orbit governed by the coset representation $\left(\mathbf{C}_{3 \widehat{I}} \backslash\right) \mathbf{O}_{\widehat{I}}$, the resulting fixed-point vector FPVcube is identical with the 17 th row of the standard mark table MarkTableOI_cube (and the 17th row of the corresponding matrix Matrix_tomOI_cube) of the $L R$-permutation group $\mathbf{O}_{\widehat{I}}$. Note that each position of the holantimeric cubane skeleton $\overline{2}$ (D) exhibits its local symmetry $\mathbf{C}_{3 \widehat{I}}$ under the action of $L R$-permutation group $\mathbf{O}_{\widehat{I}}$. Strictly speaking, the above discussions are concerned with the pair of holantimeric skeletons $\mathbf{1} / \overline{\mathbf{2}}$, which appears in a diagonal direction and is governed by the $L R$-permutation group $\mathbf{O}_{\widehat{I}}$.

This conclusion is confirmed in terms of the multiplicity of orbits as a multiplicity vector orbit_OI_cube, which is calculated by the multiplication of the FPV (FPVcube) with the inverse matrix of the mark table Inverse(Matrix_tomOI_cube) as follows:

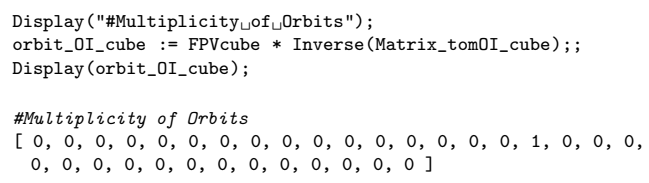

The value 1 at the 17th position of the list orbit_OI_cube represents the appearance of one orbit governed by the coset representation $\left(\mathbf{C}_{3 \widehat{I}} \backslash\right) \mathbf{O}_{\widehat{I}}$.

The SCI-CFs for a cubane skeleton under $L R$-permutation group $\mathbf{O}_{\widehat{I}}$ are obtained by referring the multiplicity vector orbit_OI_cube. Because it contains the value 1 at the 17th position, the corresponding list of SCI-CFs 1_SCICF_OI_cube is occasionally concerned with only one orbit, i.e., the 17 th inner list of the above simplified citation of the standard USCI table USCITableOI_cube. According to the procedure of general multipleorbit cases, the newly-developed function constructSCICF can be used to calculate a list of SCI-CFs 1_SCICF_OI_cube, even in this one-orbit case.

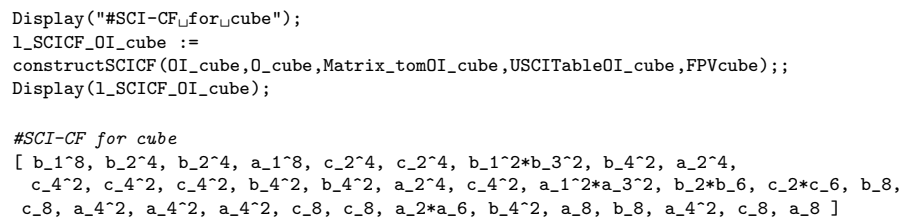

The resulting list of SCI-CFs 1_SCICF_OI_cube is multiplied by the inverse matrix of the mark table Inverse (Matrix_tomOI_cube), so as to give a list of PCI-CFs. 
Display ("\#list of $_{\sqcup} P C I-C F s \_$for $r_{\sqcup}$ cube") ;

1_PCICF_OI_cube := 1_SCICF_OI_cube * Inverse (Matrix_tomOI_cube); ;

Display (1_PCICF_OI_cube);

The list of PCI-CFs 1_PCICF_OI_cube contains PCI-CFs for thirty-three subgroups, each of which is represented as 1_PCICF_OI_cube [i] $(i=1,2, \ldots, 33)$ and taken out as a polynomial expression.

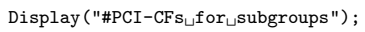

for $i$ in [1...33] do

Print ("PCICF_OI_cube [", i, "] od;

\section{$\# P C I$-CFs for subgroups}

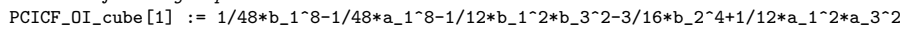

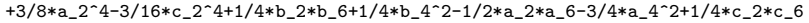

$+1 / 2 * c_{-} 4 * 2-1 / 4 * b_{-} 8+1 / 2 * a_{-} 8-1 / 4 * c_{-} 8$

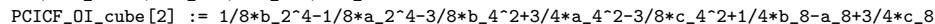

PCICF_OI_cube [3] : : $1 / 4 * b_{-} 2 \sim 4-1 / 4 * a_{-} 2 \sim 4-1 / 2 * b_{-} 2 * b_{-}-6-1 / 4 * b_{-} 4 \sim 2+1 / 2 * a_{-} 2 * a_{-} 6+1 / 2 * a_{-} 4{ }^{\sim} 2$

$-1 / 4 * c_{-} 4{ }^{-} 2+1 / 2 * b_{-} 8-1 / 2 * a_{-} 8$

PCICF_OI_cube [4] := 1/24*a_1^8-1/6*a_1^2*a_3^2-3/8*a_2^4+1/2*a_2*a_6+1/2*a_4^2-1/2*a_8

PCICF_OI_cube $[5]:=-1 / 8 * a_{-} 2 \sim 4+1 / 8 * c_{-} 2 \sim 4+1 / 2 * a_{-} 44^{\wedge} 2-1 / 2 * c_{-} 4 \wedge 2$

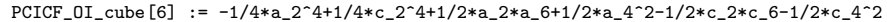

$-1 / 2 * a_{-} 8+1 / 2 * c_{-} 8$

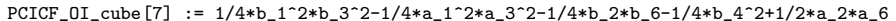

$+1 / 4 * a_{-} 4-2-1 / 4 * c_{-} 2 * c_{-} 6+1 / 4 * b_{-}-8-1 / 2 * a_{-} 8+1 / 4 * c_{-} 8$

PCICF_OI_cube [8] : $=0$

PCICF_OI_cube [9] := 1/4*a_2^4-3/4*a_4^2+1/2*a_8

PCICF_OI_cube [10] : $=-1 / 4 * \mathrm{a}_{4} 4 \sim 2+1 / 4 * \mathrm{c}_{-} 4 \sim 2+1 / 2 * \mathrm{a}_{-} 8-1 / 2 * \mathrm{c}_{-} 8$

PCICF_OI_cube [11] := -1/4*a_4^2+1/4*c_4^ $2+1 / 2 * a_{-}-8-1 / 2 * c_{-} 8$

PCICF_OI_cube [12] : : $-1 / 4 * a_{-} 44^{\wedge} 2+1 / 4 * c_{-} 4 \wedge 2+1 / 2 * a_{-} 8-1 / 2 * c_{-} 8$

PCICF_OI_cube [13] $:=1 / 4 * b_{-} 4 \sim 2-1 / 4 * a_{-} 4 \wedge 2-1 / 4 * b_{-} 8+1 / 2 * a \_8-1 / 4 * c \_8$

PCICF_OI_cube [14] := 1/4*b_4^2-1/4*a_4^2-1/4*b_8+1/2*a_8-1/4*c_8

PCICF_OI_cube [15] $:=1 / 2 * a_{-} 2 \sim 4-a_{-} 2 * a_{-} 6-1 / 2 * a_{-} 4 * 2+a_{-} 8$

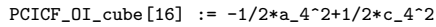

PCICF_OI_cube [17] : : $1 / 2 * a_{-} 1{ }^{\wedge} 2 * a_{-} 3 \sim 2-1 / 2 * a_{-} 2 * a_{-} 6-1 / 2 * a_{-} 4{ }^{-} 2+1 / 2 * a_{-} 8$

PCICF_OI_cube [18] $:=1 / 2 * \mathrm{~b}_{-} 2 * \mathrm{~b} \_6-1 / 2 * \mathrm{a}_{-} 2 * \mathrm{a} \_6-1 / 2 * \mathrm{~b} \_8+1 / 2 * \mathrm{a}$ _ 8

PCICF_OI_cube [19] : $=-1 / 2 * a_{-} 2 * a_{-} 6+1 / 2 * c_{-} 2 * c_{-} 6+1 / 2 * a_{-} 8-1 / 2 * c_{-} 8$

PCICF_OI_cube $[20]:=0$

PCICF_OI_cube [21] : : 0

PCICF_OI_cube $[22]:=1 / 2 * a_{-} 4 \sim 2-1 / 2 * a_{-} 8$

PCICF_OI_cube [23] := $1 / 2 * a_{-} 4 \sim 2-1 / 2 * a_{-} 8$

PCICF_OI_cube $[24]:=0$

PCICF_OI_cube $[25]:=-1 / 2 * a_{-} 8+1 / 2 * c_{-} 8$

PCICF_OI_cube $[26]:=-1 / 2 * a_{-} 8+1 / 2 * c_{-} 8$

PCICF_OI_cube $[27]:=a_{-} 2 * a_{-} 6-a_{-} 8$

PCICF_OI_cube [28] := 1/4*b_4 ${ }^{\sim} 2-1 / 4 * a_{-} 4{ }^{*} 2-1 / 4 * b_{-} 8+1 / 2 * a_{-} 8-1 / 4 * c_{-} 8$

PCICF_OI_cube [29] $:=0$

PCICF_OI_cube [30] : $=1 / 2 * b_{-} 8-1 / 2 * a \_8$

PCICF_OI_cube $[31]:=1 / 2 * a_{-} 4-2-1 / 2 * a_{-} 8$

PCICF_OI_cube [32] : $=-1 / 2 * a_{-} 8+1 / 2 * c_{-} 8$

PCICF_OI_cube $[33]:=a_{-} 8$

These PCI-CFs for the $L R$-permutation group $\mathbf{O}_{\widehat{I}}$ in the GAP expressions are represented by usual mathematic conventions as follows:

$$
\begin{aligned}
\text { PCI-CF }_{\mathbf{O}_{\widehat{I}} \mathbf{1}}\left(\mathbf{C}_{1}, \$_{d}\right)= & \frac{1}{48} b_{1}^{8}-\frac{1}{48} a_{1}^{8}-\frac{1}{12} b_{1}^{2} b_{3}^{2}-\frac{3}{16} b_{2}^{4}+\frac{1}{12} a_{1}^{2} a_{3}^{2} \\
& +\frac{3}{8} a_{2}^{4}-\frac{3}{16} c_{2}^{4}+\frac{1}{4} b_{2} b_{6}+\frac{1}{4} b_{4}^{2}-\frac{1}{2} a_{2} a_{6}-\frac{3}{4} a_{4}^{2}+\frac{1}{4} c_{2} c_{6} \\
& +\frac{1}{2} c_{4}^{2}-\frac{1}{4} b_{8}+\frac{1}{2} a_{8}-\frac{1}{4} c_{8} \\
\text { PCI-CF }_{\mathbf{O}_{\hat{I}} \mathbf{1}}\left(\mathbf{C}_{2}, \$_{d}\right)= & \frac{1}{8} b_{2}^{4}-\frac{1}{8} a_{2}^{4}-\frac{3}{8} b_{4}^{2}+\frac{3}{4} a_{4}^{2}-\frac{3}{8} c_{4}^{2}+\frac{1}{4} b_{8}-a_{8}+\frac{3}{4} c_{8}
\end{aligned}
$$




$$
\begin{aligned}
& \text { PCI-CF } \mathbf{O}_{\hat{I}} \mathbf{1}\left(\mathbf{C}_{2}^{\prime}, \$_{d}\right)=\frac{1}{4} b_{2}^{4}-\frac{1}{4} a_{2}^{4}-\frac{1}{2} b_{2} b_{6}-\frac{1}{4} b_{4}^{2} \\
& +\frac{1}{2} a_{2} a_{6}+\frac{1}{2} a_{4}^{2}-\frac{1}{4} c_{4}^{2}+\frac{1}{2} b_{8}-\frac{1}{2} a_{8} \\
& \text { PCI-CF } \mathbf{O}_{\hat{I}} \mathbf{1}\left(\mathbf{C}_{\widehat{I}}, \$_{d}\right)=\frac{1}{24} a_{1}^{8}-\frac{1}{6} a_{1}^{2} a_{3}^{2}-\frac{3}{8} a_{2}^{4}+\frac{1}{2} a_{2} a_{6}+\frac{1}{2} a_{4}^{2}-\frac{1}{2} a_{8} \\
& \text { PCI-CF } \mathbf{O}_{\hat{I}} \mathbf{1}\left(\widehat{\mathbf{C}}_{2}, \$_{d}\right)=-\frac{1}{8} a_{2}^{4}+\frac{1}{8} c_{2}^{4}+\frac{1}{2} a_{4}^{2}-\frac{1}{2} c_{4}^{2} \\
& \text { PCI-CF } \mathbf{O}_{\hat{I}} \mathbf{1}\left(\widehat{\mathbf{C}}_{2}^{\prime}, \$_{d}\right)=-\frac{1}{4} a_{2}^{4}+\frac{1}{4} c_{2}^{4}+\frac{1}{2} a_{2} a_{6}+\frac{1}{2} a_{4}^{2}-\frac{1}{2} c_{2} c_{6}-\frac{1}{2} c_{4}^{2}-\frac{1}{2} a_{8}+\frac{1}{2} c_{8}(81) \\
& \text { PCI-CF } \mathbf{O}_{\hat{I}} \mathbf{1}\left(\mathbf{C}_{3}, \$_{d}\right)=\frac{1}{4} b_{1}^{2} b_{3}^{2}-\frac{1}{4} a_{1}^{2} a_{3}^{2}-\frac{1}{4} b_{2} b_{6}-\frac{1}{4} b_{4}^{2} \\
& +\frac{1}{2} a_{2} a_{6}+\frac{1}{4} a_{4}^{2}-\frac{1}{4} c_{2} c_{6}+\frac{1}{4} b_{8}-\frac{1}{2} a_{8}+\frac{1}{4} c_{8} \\
& \text { PCI-CF } \mathbf{O}_{\hat{I}} \mathbf{1}\left(\mathbf{C}_{2 \widehat{I}}, \$_{d}\right)=\frac{1}{4} a_{2}^{4}-\frac{3}{4} a_{4}^{2}+\frac{1}{2} a_{8} \\
& \text { PCI-CF } \mathbf{O}_{\hat{I}} \mathbf{1}\left(\widehat{\mathbf{D}}_{2}, \$_{d}\right)=-\frac{1}{4} a_{4}^{2}+\frac{1}{4} c_{4}^{2}+\frac{1}{2} a_{8}-\frac{1}{2} c_{8} \\
& \text { PCI-CF } \mathbf{O}_{\hat{I}} \mathbf{1}\left(\widehat{\mathbf{C}}_{4}, \$_{d}\right)=-\frac{1}{4} a_{4}^{2}+\frac{1}{4} c_{4}^{2}+\frac{1}{2} a_{8}-\frac{1}{2} c_{8} \\
& \text { PCI-CF } \mathbf{O}_{\hat{I}} \mathbf{1}\left(\widehat{\mathbf{D}}_{2}^{\prime}, \$_{d}\right)=-\frac{1}{4} a_{4}^{2}+\frac{1}{4} c_{4}^{2}+\frac{1}{2} a_{8}-\frac{1}{2} c_{8} \\
& \text { PCI-CF } \mathbf{O}_{\hat{I}} \mathbf{1}\left(\mathbf{C}_{4}, \$_{d}\right)=\frac{1}{4} b_{4}^{2}-\frac{1}{4} a_{4}^{2}-\frac{1}{4} b_{8}+\frac{1}{2} a_{8}-\frac{1}{4} * c_{8} \\
& \text { PCI-CF } \mathbf{O}_{\hat{I}} \mathbf{1}_{1}\left(\mathbf{D}_{2}^{\prime}, \$_{d}\right)=\frac{1}{4} b_{4}^{2}-\frac{1}{4} a_{4}^{2}-\frac{1}{4} b_{8}+\frac{1}{2} a_{8}-\frac{1}{4} c_{8} \\
& \text { PCI-CF } \mathbf{O}_{\hat{I}} \mathbf{1}\left(\mathbf{C}_{2 \widehat{I}}^{\prime}, \$_{d}\right)=\frac{1}{2} a_{2}^{4}-a_{2} a_{6}-\frac{1}{2} a_{4}^{2}+a_{8} \\
& \text { PCI-CF } \mathbf{O}_{\hat{I}} \mathbf{1}\left(\widehat{\mathbf{D}}_{2}^{\prime \prime}, \$_{d}\right)=-\frac{1}{2} a_{4}^{2}+\frac{1}{2} c_{4}^{2} \\
& \text { PCI-CF } \mathbf{O}_{\hat{I}} \mathbf{1}\left(\mathbf{C}_{3 \hat{I}}, \$_{d}\right)=\frac{1}{2} a_{1}^{2} a_{3}^{2}-\frac{1}{2} a_{2} a_{6}-\frac{1}{2} a_{4}^{2}+\frac{1}{2} a_{8} \\
& \text { PCI-CF } \mathbf{O}_{\hat{I}} \mathbf{1}\left(\mathbf{D}_{3}, \$_{d}\right)=\frac{1}{2} b_{2} b_{6}-\frac{1}{2} a_{2} a_{6}-\frac{1}{2} b_{8}+\frac{1}{2} a_{8} \\
& \text { PCI-CF } \mathbf{O}_{\hat{I}} \mathbf{1}\left(\widehat{\mathbf{D}}_{3}, \$_{d}\right)=-\frac{1}{2} a_{2} a_{6}+\frac{1}{2} c_{2} c_{6}+\frac{1}{2} a_{8}-\frac{1}{2} c_{8} \\
& \text { PCI-CF } \mathbf{O}_{\hat{I}} \mathbf{1}\left(\mathbf{D}_{4}, \$_{d}\right)=0 \\
& \text { PCI-CF }{ }_{\mathbf{O}_{\hat{I}}} \mathbf{1}\left(\widehat{\mathbf{D}}_{4}, \$_{d}\right)=0 \\
& \text { PCI-CF } \text { O }_{\hat{I}} \mathbf{1}\left(\mathbf{C}_{4 \widehat{I}}, \$_{d}\right)=\frac{1}{2} a_{4}^{2}-\frac{1}{2} a_{8} \\
& \text { PCI-CF } \mathbf{O}_{\hat{I}} \mathbf{1}\left(\mathbf{D}_{2 \widehat{I}}^{\prime \prime}, \$_{d}\right)=\frac{1}{2} a_{4}^{2}-\frac{1}{2} a_{8} \\
& \text { PCI-CF } \mathbf{O}_{\hat{I}}\left(\mathbf{D}_{2 \widehat{I}}, \$_{d}\right)=0
\end{aligned}
$$




$$
\begin{aligned}
& \text { PCI-CF } \mathbf{O}_{\widehat{I}} \mathbf{1}\left(\widehat{\mathbf{D}}_{4}^{\prime \prime}, \$_{d}\right)=-\frac{1}{2} a_{8}+\frac{1}{2} c_{8} \\
& \text { PCI-CF } \mathbf{O}_{\widehat{I}} \mathbf{1}\left(\widehat{\mathbf{D}}_{4}^{\prime}, \$_{d}\right)=-\frac{1}{2} a_{8}+\frac{1}{2} c_{8} \\
& \text { PCI-CF } \mathbf{O}_{\widehat{I}} \mathbf{1}\left(\mathbf{D}_{3 \widehat{I}}, \$_{d}\right)=a_{2} a_{6}-a_{8} \\
& \text { PCI-CF } \mathbf{O}_{\widehat{I}} \mathbf{1}\left(\mathbf{T}, \$_{d}\right)=\frac{1}{4} b_{4}^{2}-\frac{1}{4} a_{4}^{2}-\frac{1}{4} b_{8}+\frac{1}{2} a_{8}-\frac{1}{4} c_{8} \\
& \text { PCI-CF } \mathbf{O}_{\widehat{I}} \mathbf{1}\left(\mathbf{D}_{4 \widehat{I}}, \$_{d}\right)=0 \\
& \text { PCI-CF } \mathbf{O}_{\widehat{I}} \mathbf{1}\left(\mathbf{O}, \$_{d}\right)=\frac{1}{2} b_{8}-\frac{1}{2} a_{8} \\
& \text { PCI-CF } \mathbf{O}_{\widehat{I}} \mathbf{1}\left(\mathbf{T}_{\widehat{I}}, \$_{d}\right)=\frac{1}{2} a_{4}^{2}-\frac{1}{2} a_{8} \\
& \text { PCI-CF } \mathbf{O}_{\widehat{I}} \mathbf{1}\left(\widehat{\mathbf{O}}, \$_{d}\right)=-\frac{1}{2} a_{8}+\frac{1}{2} c_{8} \\
& \text { PCI-CF } \mathbf{O}_{\widehat{I}} \mathbf{1}\left(\mathbf{O}_{\widehat{I}}, \$_{d}\right)=a_{8}
\end{aligned}
$$

\section{Combinatorial enumerations}

Fujita's USCI approach supports four methods of combinatorial enumeration, i.e., (1) the fixed-point matrix (FPM) method based on generating functions derived from subduced cycle indices (SCIs) and mark tables [32-34], (2) the partial-cycle-index (PCI) method based on generating functions derived from partial cycle indices (PCIs) [35, 36], (3) the elementary superposition method [37], and (4) the partial superposition method [35,37]. Among the four methods, the present paper adopts the partial-cycle-index (PCI) method, where the use of CPR (combined-permutation representation) under the GAP system is a new matter.

\subsection{Symmetry-itermized enumeration under point group $\mathrm{O}_{h}$}

The PCI-CFs calculated under the point group $\mathbf{O}_{h}$ (Eqs. 8-40) are used in symmetryitemized enumeration of cubane derivatives under the point group $\mathbf{O}_{h}$. For the sake of simplicity, a set of eight proligands is selected from the following inventory of proligands:

$$
\mathbf{L}=\{\mathrm{H}, \mathrm{A}, \mathrm{B}, \mathrm{X}, \mathrm{Y}, \mathrm{Z} ; \mathrm{p}, \overline{\mathrm{p}} ; \mathrm{q}, \overline{\mathrm{q}}\}
$$

where $\mathrm{H}, \mathrm{A}, \mathrm{B}, \mathrm{X}, \mathrm{Y}$, and $\mathrm{Z}$ are achiral proligands in isolation, while a pair of $\mathrm{p}$ and $\overline{\mathrm{p}}$ (or $\mathrm{q}$ and $\overline{\mathrm{q}}$ ) represents an enantiomeric pair of chiral proligands in isolation. The corresponding 
ligand-inventory functions are obtained according to Eqs. 5-7 in Theorem 1 of Ref. [38].

$$
\begin{aligned}
& a_{d}=\mathrm{H}^{d}+\mathrm{A}^{d}+\mathrm{B}^{d}+\mathrm{X}^{d}+\mathrm{Y}^{d}+\mathrm{Z}^{d} \\
& b_{d}=\mathrm{H}^{d}+\mathrm{A}^{d}+\mathrm{B}^{d}+\mathrm{X}^{d}+\mathrm{Y}^{d}+\mathrm{Z}^{d}+\mathrm{p}^{d}+\overline{\mathrm{p}}^{d}+\mathrm{q}^{d}+\overline{\mathrm{q}}^{d} \\
& c_{d}=\mathrm{H}^{d}+\mathrm{A}^{d}+\mathrm{B}^{d}+\mathrm{X}^{d}+\mathrm{Y}^{d}+\mathrm{Z}^{d}+2 \mathrm{p}^{d / 2} \overline{\mathrm{p}}^{d / 2}+2 \mathrm{q}^{d / 2} \overline{\mathrm{q}}^{d / 2}
\end{aligned}
$$

The ligand-inventory functions (Eqs. 110-112) are introduced into the PCI-CFs (Eqs. 8-40). The resulting equation is expanded to give a generating function, in which the coefficient of each term $\mathrm{H}^{h} \mathrm{~A}^{a} \mathrm{~B}^{b} \mathrm{X}^{x} \mathrm{Y}^{y} \mathrm{Z}^{z} \mathrm{p}^{p} \overline{\mathrm{p}}^{\bar{p}} \mathrm{q}^{q} \overline{\mathrm{q}}^{\bar{q}}$ represents the number of pairs of enantiomeric cubane derivatives with the composition $\mathrm{C}_{8} \mathrm{H}_{h} \mathrm{~A}_{a} \mathrm{~B}_{b} \mathrm{X}_{x} \mathrm{Y}_{y} \mathrm{Z}_{z} \mathrm{p}_{p} \overline{\mathrm{p}}_{\bar{p}} \mathrm{q}_{q} \overline{\mathrm{q}}_{\bar{q}}$.

Because the coefficients appear symmetrically, such a mode of substitution is represented by a substitution pattern $[h, a, b, x, y, z ; p, \bar{p}, q, \bar{q}]$ for the sake of convenience in enumeration. The symmetrical appearance permits us to presume $h \geq a \geq w \geq x \geq y \geq z$; $p \geq q, p \geq \bar{p}$, and $q \geq \bar{q}$ without losing generality. Appendix A shows a typical procedure for Fujita's USCI approach by using the combined-permutation representation (CPR). The coefficients obtained are collected in a tabular form (Tables 1 to 3 ).

Because a pair of enantiomers is counted once in the enumeration under the point group $\mathbf{O}_{h}$, a coefficient should be duplicated if there is a pair of terms which corresponds to a pair of enantiomers (each substitution pattern with an asterisk). Each substitution pattern marked by an asterisk (e.g., $[7,0,0,0,0,0 ; 1,0,0,0]^{*}$ for $\mathrm{H}^{7} \mathrm{p}$ ) has the counterpart of opposite chirality sense (e.g., $[7,0,0,0,0,0 ; 0,1,0,0]^{*}$ for $\mathrm{H}^{7} \overline{\mathrm{p}}$ ), so that the corresponding coefficient should be duplicated to generate the number of cubane derivatives.

The symmetry-itemized enumeration has been conducted by means of the partialcycle-index (PCI) method of Fujita's USCI approach (Appendix A), where the nonredundant set of subgroups $\mathrm{SSG}_{\mathrm{O}_{h}}$ (Eq. 7) has been postulated. The resulting data are collected in Tables 1 to 3 . These data are consistent with the previous results which have been obtained by applying the fixed-point matrix (FPM) method of Fujita's USCI approach [39], the elementary-superposition method of Fujita's USCI approach [21], as well as the partial-cycle-index (PCI) method of Fujita's USCI approach [20]. Among them, the last report deals with the PCI method, but it has been conducted under the Maple programming language in place of the GAP system and with no use of CPR (combinedpermutation representation). 
Table 1. Cubane Derivatives as 3D Structural Isomers Under the Point Group $\mathbf{O}_{h}$ (Part 1)

\begin{tabular}{|c|c|c|c|c|c|c|c|c|c|c|c|c|c|c|c|c|c|c|c|c|}
\hline partition & $\begin{array}{l}\mathbf{C}_{1} \\
\mathrm{C}_{4}\end{array}$ & & & & $\mathrm{C}_{s}^{\prime}$ & & $\begin{array}{c}\mathrm{C}_{3} \\
\mathrm{~T}\end{array}$ & & S & & $\begin{array}{l}\mathbf{D}_{2}^{\prime} \\
\mathbf{T}_{h}\end{array}$ & $\mathbf{T}_{d}$ & & & & $\mathrm{C}_{2 h}^{\prime}$ & $\mathrm{D}_{3}$ & & & $\mathrm{D}_{4}$ \\
\hline \multirow[t]{2}{*}[8,0,0,0,0,0,0,0,0,0]{} & 0 & 0 & 0 & 0 & 0 & 0 & 0 & 0 & 0 & 0 & 0 & 0 & 0 & 0 & 0 & 0 & 0 & 0 & 0 & 0 \\
\hline & 0 & 0 & 0 & 0 & 0 & 0 & 0 & & 0 & 0 & 0 & 0 & 1 & & & & & & & \\
\hline \multirow[t]{2}{*}[7,1,0,0,0,0,0,0,0,0]{} & 0 & 0 & 0 & 0 & 0 & 0 & 0 & 0 & 0 & 0 & 0 & 0 & 0 & 0 & 0 & 0 & 0 & 1 & 0 & 0 \\
\hline & 0 & 0 & 0 & 0 & 0 & 0 & 0 & 0 & 0 & 0 & 0 & 0 & 0 & & & & & & & \\
\hline \multirow[t]{2}{*}[6,2,0,0,0,0,0,0,0,0]{} & 0 & 0 & 0 & 0 & 0 & 0 & 0 & 0 & 0 & 0 & 0 & 0 & 1 & 1 & 0 & 0 & 0 & 0 & 0 & 0 \\
\hline & 0 & 0 & 0 & 0 & 0 & 0 & 0 & 1 & 0 & 0 & 0 & 0 & 0 & & & & & & & \\
\hline \multirow[t]{2}{*}[6,1,1,0,0,0,0,0,0,0]{} & 0 & 0 & 0 & 0 & 2 & 0 & 0 & 0 & 0 & 0 & 0 & 0 & 0 & 0 & 0 & 0 & 0 & 1 & 0 & 0 \\
\hline & 0 & 0 & 0 & 0 & 0 & 0 & 0 & 0 & 0 & 0 & 0 & 0 & 0 & & & & & & & \\
\hline \multirow{2}{*}[5,3,0,0,0,0,0,0,0,0]{} & 0 & 0 & 0 & 0 & 2 & 0 & 0 & 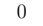 & 0 & 0 & 0 & 0 & 0 & 0 & 0 & 0 & 0 & 1 & 0 & 0 \\
\hline & 0 & 0 & 0 & 0 & 0 & 0 & 0 & 0 & 0 & 0 & 0 & 0 & 0 & & & & & & & \\
\hline \multirow{2}{*}[5,2,1,0,0,0,0,0,0,0]{} & 1 & 0 & 0 & 0 & 5 & 0 & 0 & 0 & 0 & 0 & 0 & 0 & 0 & 0 & 0 & 0 & 0 & 0 & 0 & 0 \\
\hline & 0 & 0 & 0 & 0 & 0 & 0 & 0 & 0 & 0 & 0 & 0 & 0 & 0 & & & & & & & \\
\hline \multirow{2}{*}[5,1,1,1,0,0,0,0,0,0]{} & 4 & 0 & 0 & 0 & 6 & 0 & 0 & 0 & 0 & 0 & 0 & 0 & 0 & 0 & 0 & 0 & 0 & 0 & 0 & 0 \\
\hline & 0 & 0 & 0 & 0 & 0 & 0 & 0 & 0 & 0 & 0 & 0 & 0 & 0 & & & & & & & \\
\hline \multirow{2}{*}[4,4,0,0,0,0,0,0,0,0]{} & 0 & 0 & 1 & 0 & 1 & 0 & 0 & 0 & 0 & 0 & 0 & 0 & 0 & 0 & 0 & 0 & 0 & 1 & 0 & 0 \\
\hline & 1 & 0 & 0 & 0 & 0 & 1 & 0 & & 0 & 0 & 0 & 1 & 0 & & & & & & & \\
\hline$[4,3,1,0,0,0,0,0,0,0]$ & 3 & 0 & 0 & 0 & 5 & 0 & 0 & & 0 & 0 & 0 & 促 & 0 & 0 & 0 & 0 & 0 & 2 & 0 & 0 \\
\hline & 0 & 0 & 0 & 0 & 0 & 0 & 0 & 0 & 0 & 0 & 0 & 0 & 0 & & & & & & & \\
\hline$[4,2$, & 4 & 0 & 2 & 1 & 4 & 0 & 0 & 0 & 0 & 0 & 0 & 0 & 3 & 1 & 0 & 1 & 0 & 0 & 0 & 0 \\
\hline & 0 & 0 & 0 & 0 & 0 & 0 & 0 & 0 & 0 & 0 & 0 & 0 & 0 & & & & & & & \\
\hline$[4,2,1,1,0,0,0,0,0,0]$ & 13 & 0 & 0 & 0 & 9 & 0 & 0 & 0 & 0 & 0 & 0 & 0 & 0 & 0 & 0 & 0 & 0 & 0 & 0 & 0 \\
\hline & 0 & 0 & 0 & 0 & 0 & 0 & 0 & & 0 & 0 & 0 & 0 & 0 & & & & & & & \\
\hline$[4,1,1,1,1,0,0,0,0,0]$ & 32 & 0 & 0 & 0 & 6 & 0 & 0 & 0 & 0 & 0 & 0 & 0 & 0 & 0 & 0 & 0 & 0 & 0 & 0 & 0 \\
\hline & 0 & 0 & 0 & 0 & 0 & 0 & 0 & 0 & 0 & 0 & 0 & 0 & 0 & & & & & & & \\
\hline$, 0,0,0,0,0,0]$ & 7 & 0 & 0 & 0 & 9 & 0 & 0 & 0 & 0 & 0 & 0 & 0 & 0 & 0 & 0 & 0 & 0 & 1 & 0 & 0 \\
\hline & 0 & 0 & 0 & 0 & 0 & 0 & O & & 0 & 0 & 0 & 0 & 0 & & & & & & & \\
\hline$[3,3,1,1,0,0,0,0,0,0]$ & 18 & 0 & 0 & 0 & 10 & 0 & 0 & 0 & 0 & 0 & 0 & 0 & 0 & 0 & 0 & 0 & 0 & 2 & 0 & 0 \\
\hline & 0 & 0 & o & 0 & 0 & 0 & 0 & & 0 & 0 & 0 & 0 & 0 & & & & & & & \\
\hline$[3,2,2,1,0,0,0,0,0,0]$ & 28 & 0 & 0 & 0 & 14 & 0 & 0 & 0 & 0 & 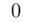 & 0 & 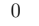 & 0 & 0 & 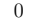 & 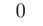 & 0 & 0 & 0 & 0 \\
\hline & 0 & 0 & 0 & 0 & 0 & 0 & 0 & 0 & 0 & 0 & 0 & 0 & 0 & & & & & & & \\
\hline$[3,2,1,1,1,0,0,0,0,0]$ & 64 & 0 & 0 & 0 & 12 & 0 & 0 & 0 & 0 & 0 & 0 & 0 & 0 & 0 & 0 & 0 & 0 & 0 & 0 & 0 \\
\hline & 0 & 0 & 0 & 0 & 0 & 0 & 0 & & 0 & 0 & 0 & . & 0 & & & & & & & \\
\hline$[2,2,2,2,0,0,0,0,0,0]$ & 40 & 0 & 6 & 3 & 12 & 1 & 0 & c & 0 & 0 & 0 & 0 & 6 & 0 & 0 & 0 & 0 & 0 & 8 & 0 \\
\hline & 0 & 0 & 0 & 0 & 0 & 0 & 0 & & 0 & 0 & 0 & 0 & 0 & & & & & & & \\
\hline$[2,2$, & 96 & 0 & 0 & 0 & 18 & 0 & 0 & 0 & 0 & 0 & 0 & 0 & 0 & 0 & 0 & 0 & 0 & 0 & 0 & 0 \\
\hline & 0 & 0 & 0 & 0 & 0 & 0 & 0 & 0 & 0 & 0 & 0 & 0 & 0 & & & & & & & \\
\hline$[2,2$, & 204 & 0 & 0 & 0 & 12 & 0 & 0 & 0 & 0 & 0 & 0 & 0 & 0 & 0 & 0 & 0 & 0 & 0 & 0 & 0 \\
\hline & 0 & 0 & 0 & 0 & 0 & 0 & 0 & 0 & 0 & 0 & 0 & 0 & 0 & & & & & & & \\
\hline$[7,0,0,0,0,0,1,0,0,0]^{*}$ & 0 & 0 & 0 & 0 & P & 0 & $1 / 2$ & 0 & 0 & 0 & 0 & 0 & 0 & 0 & 0 & 8 & 0 & 0 & 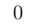 & 0 \\
\hline & 0 & 0 & 0 & 0 & 0 & 0 & & 0 & 0 & 0 & 0 & 0 & 0 & & & & & & & \\
\hline $0,0,1,0,0,0]^{*}$ & 1 & 0 & 0 & 0 & 0 & 0 & $1 / 2$ & 0 & 0 & 0 & 0 & 0 & 0 & 0 & 0 & 0 & 0 & 0 & 0 & 0 \\
\hline & 0 & 0 & 0 & 0 & 0 & 0 & 0 & 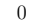 & 0 & 0 & 0 & 0 & 0 & & & & & & & \\
\hline$[6,0,0,0,0,0,2,0,0,0]^{*}$ & 0 & $1 / 2$ & $1 / 2$ & 0 & 0 & 0 & 0 & 0 & 0 & 0 & 0 & 0 & 0 & 0 & 0 & 0 & $1 / 2$ & 0 & 0 & 0 \\
\hline & 0 & 0 & 0 & 0 & 0 & 0 & 0 & 0 & 0 & 0 & 0 & 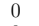 & 0 & & & & & & & \\
\hline$[6,0$, & 0 & 0 & 0 & 1 & 1 & 0 & 0 & 0 & 0 & 0 & 0 & 0 & 0 & 0 & 0 & 0 & 0 & 0 & 1 & 0 \\
\hline & 0 & 0 & 0 & 0 & 0 & 0 & 0 & 0 & 0 & 0 & 0 & 0 & 0 & & & & & & & \\
\hline$, 0,1,0,0,0]^{*}$ & $7 / 2$ & 0 & 0 & 0 & 0 & 0 & 0 & U & 0 & 0 & 0 & 0 & 0 & 0 & 0 & 0 & 0 & 0 & 0 & 0 \\
\hline & 0 & 0 & 0 & 0 & 0 & 0 & 0 & 0 & 0 & 0 & 0 & 0 & 0 & & & & & & & \\
\hline$[5,1,1,0,0,0,1,0,0,0]^{*}$ & 7 & 0 & 0 & 0 & 0 & 0 & 0 & 0 & 0 & 0 & 0 & 0 & 0 & 0 & 0 & 0 & 0 & 0 & 0 & 0 \\
\hline & 0 & O & 0 & 0 & 0 & 0 & 0 & 0 & 0 & 0 & 0 & 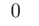 & 0 & & & & & & & \\
\hline & $7 / 2$ & 0 & 0 & 0 & 0 & 0 & 0 & 0 & 0 & 0 & 0 & 0 & 0 & 0 & 0 & 0 & 0 & 0 & 0 & 0 \\
\hline & 0 & 0 & 0 & 0 & 0 & 0 & 0 & 0 & 0 & 0 & 0 & 0 & 0 & & & & & & & \\
\hline$[5$ & 5 & 0 & 0 & 0 & 4 & 0 & 0 & 0 & 0 & 0 & 0 & 0 & 0 & 0 & 0 & 0 & 0 & 0 & 0 & 0 \\
\hline & 0 & 0 & 0 & 0 & 0 & 0 & 0 & 0 & 0 & 0 & 0 & 0 & 0 & & & & & & & \\
\hline$[5,0,0,0,0,0,3,0,0,0]^{*}$ & 1 & 0 & 0 & 0 & 0 & 0 & $1 / 2$ & 0 & 0 & 0 & 0 & 0 & 0 & 0 & 0 & 0 & 0 & 0 & 0 & 0 \\
\hline & 0 & 0 & 0 & 0 & 0 & 0 & 0 & 0 & 0 & 0 & 0 & 0 & 0 & & & & & & & \\
\hline$[5,0$ & $7 / 2$ & 0 & 0 & 0 & 0 & 0 & 0 & 0 & 0 & 0 & 0 & 0 & 0 & 0 & 0 & 0 & 0 & 0 & 0 & 0 \\
\hline & 0 & 0 & 0 & 0 & 0 & 0 & 0 & 0 & 0 & 0 & 0 & 0 & 0 & & & & & & & \\
\hline$[5,0,0,0,0,0,1,1,1,0]^{*}$ & 7 & 0 & 0 & 0 & 0 & 0 & 0 & 0 & 0 & 0 & 0 & 0 & 0 & 0 & 0 & 0 & 0 & 0 & 0 & 0 \\
\hline & 0 & 0 & 0 & 0 & 0 & 0 & 0 & 0 & 0 & 0 & 0 & 0 & 0 & & & & & & & \\
\hline
\end{tabular}


Table 2. Cubane Derivatives as 3D Structural Isomers Under the Point Group $\mathbf{O}_{h}$ (Part 2)

\begin{tabular}{|c|c|c|c|c|c|c|c|c|c|c|c|c|c|c|c|c|c|c|c|c|}
\hline partition & $\begin{array}{c}\mathbf{C}_{1} \\
\mathbf{C}_{4 v}\end{array}$ & $\begin{array}{c}\mathrm{C}_{2} \\
\mathrm{C}_{4 h}\end{array}$ & $\begin{array}{c}\mathrm{C}_{2}^{\prime} \\
\mathbf{D}_{2 d}\end{array}$ & $\begin{array}{c}\mathbf{C}_{s} \\
\mathbf{D}_{2 d}^{\prime}\end{array}$ & $\begin{array}{c}\mathrm{C}_{s}^{\prime} \\
\mathrm{D}_{2 h}\end{array}$ & $\begin{array}{c}\mathbf{C}_{i} \\
\mathbf{D}_{2 h}^{\prime}\end{array}$ & $\begin{array}{c}\mathrm{C}_{3} \\
\mathrm{~T}\end{array}$ & $\begin{array}{c}\mathbf{C}_{4} \\
\mathbf{D}_{3 d}\end{array}$ & $\begin{array}{c}\mathbf{S}_{4} \\
\mathbf{D}_{4 h}\end{array}$ & $\begin{array}{c}\mathrm{D}_{2} \\
\mathrm{O}\end{array}$ & $\begin{array}{l}\mathrm{D}_{2}^{\prime} \\
\mathrm{T}_{h}\end{array}$ & $\begin{array}{c}\mathbf{C}_{2 v} \\
\mathbf{T}_{d}\end{array}$ & $\begin{array}{l}\mathrm{C}_{2 v}^{\prime} \\
\mathrm{O}_{h}\end{array}$ & $\mathrm{C}_{2 v}^{\prime \prime}$ & $\mathrm{C}_{2 h}$ & $\mathbf{C}_{2 h}^{\prime}$ & $\mathbf{D}_{3}$ & $\mathbf{C}_{3 v}$ & $\mathrm{C}_{3 i}$ & $\mathrm{D}_{4}$ \\
\hline \multirow[t]{2}{*}[4,0,0,0,0,0,4,0,0,0]{$^{*}$} & $1 / 2$ & 0 & 1 & 0 & 0 & 0 & $1 / 2$ & $1 / 2$ & 0 & 0 & $1 / 2$ & 0 & 0 & 0 & 0 & 0 & 0 & 0 & 0 & 0 \\
\hline & 0 & 0 & 0 & 0 & 0 & 0 & $1 / 2$ & 0 & 0 & 0 & 0 & 0 & 0 & & & & & & & \\
\hline \multirow{2}{*}[4,3,0,0,0,0,1,0,0,0]{$^{*}$} & $11 / 2$ & 0 & 0 & 0 & 0 & 0 & 1 & 0 & 0 & 0 & 0 & 0 & 0 & 0 & 0 & 0 & 0 & 0 & 0 & 0 \\
\hline & 0 & 0 & 0 & 0 & 0 & 0 & 0 & 0 & 0 & 0 & 0 & 0 & 0 & & & & & & & \\
\hline \multirow[t]{2}{*}[4,2,0,0,0,0,2,0,0,0]{$^{*}$} & $13 / 2$ & $3 / 2$ & 3 & 0 & 0 & 0 & 0 & 0 & 0 & 0 & 0 & 0 & 0 & 0 & 0 & 0 & 0 & 0 & 0 & 0 \\
\hline & 0 & 0 & 0 & 0 & 0 & 0 & 0 & 0 & 0 & 0 & 0 & 0 & 0 & & & & & & & \\
\hline \multirow[t]{2}{*}[4,0,0,0,0,0,2,2,0,0]{} & 6 & 0 & 2 & 1 & 0 & 0 & 0 & 0 & 1 & 0 & 0 & 1 & 0 & 1 & 1 & 1 & 0 & 0 & 0 & 0 \\
\hline & 0 & 0 & 0 & 0 & 0 & 0 & 0 & 0 & 0 & 0 & 0 & 0 & 0 & & & & & & & \\
\hline \multirow{2}{*}[4,0,0,0,0,0,2,0,2,0]{$^{*}$} & $13 / 2$ & $3 / 2$ & 3 & 0 & 0 & 0 & 0 & 0 & 0 & 0 & 0 & 0 & 0 & 0 & 0 & 0 & 0 & 0 & 0 & 0 \\
\hline & 0 & 0 & 0 & 0 & 0 & 0 & 0 & 0 & 0 & 0 & 0 & 0 & 0 & & & & & & & \\
\hline \multirow[t]{2}{*}[4,2,1,0,0,0,1,0,0,0]{$^{*}$} & $35 / 2$ & 0 & 0 & 0 & 0 & 0 & 0 & 0 & 0 & 0 & 0 & 0 & 0 & 0 & 0 & 0 & 0 & 0 & 0 & 0 \\
\hline & 0 & 0 & 0 & 0 & 0 & 0 & 0 & 0 & 0 & 0 & 0 & 0 & 0 & & & & & & & \\
\hline \multirow{2}{*}[4,2,0,0,0,0,1,1,0,0]{} & 12 & 0 & 0 & 3 & 7 & 1 & 0 & 0 & 0 & 0 & 0 & 0 & 0 & 0 & 0 & 0 & 0 & 0 & 0 & 0 \\
\hline & 0 & 0 & 0 & 0 & 0 & 0 & 0 & 0 & 0 & 0 & 0 & 0 & 0 & & & & & & & \\
\hline \multirow[t]{2}{*}[4,1,1,1,0,0,1,0,0,0]{$^{*}$} & 35 & 0 & 0 & 0 & 0 & 0 & 0 & 0 & 0 & 0 & 0 & 0 & 0 & 0 & 0 & 0 & 0 & 0 & 0 & 0 \\
\hline & 0 & 0 & 0 & 0 & 0 & 0 & 0 & 0 & 0 & 0 & 0 & 0 & 0 & & & & & & & \\
\hline \multirow[t]{2}{*}[4,1,1,0,0,0,1,1,0,0]{} & 29 & 0 & 0 & 0 & 12 & 0 & 0 & 0 & 0 & 0 & 0 & 0 & 0 & 0 & 0 & 0 & 0 & 0 & 0 & 0 \\
\hline & 0 & 0 & 0 & 0 & 0 & 0 & 0 & 0 & 0 & 0 & 0 & 0 & 0 & & & & & & & \\
\hline \multirow{2}{*}[4,1,0,0,0,0,1,1,1,0]{$^{*}$} & 35 & 0 & 0 & 0 & 0 & 0 & 0 & 0 & 0 & 0 & 0 & 0 & 0 & 0 & 0 & 0 & 0 & 0 & 0 & 0 \\
\hline & 0 & 0 & 0 & 0 & 0 & 0 & 0 & 0 & 0 & 0 & 0 & 0 & 0 & & & & & & & \\
\hline$[4,0,0,0,0,0,1,1,1,1]$ & 30 & 0 & 0 & 6 & 2 & 2 & 0 & 0 & 0 & 0 & 0 & 0 & 0 & 0 & 0 & 0 & 0 & 0 & 0 & 0 \\
\hline & 0 & 0 & 0 & 0 & 0 & 0 & 0 & 0 & 0 & 0 & 0 & 0 & 0 & & & & & & & \\
\hline$[3,3,1,0,0,0,1,0,0,0]^{*}$ & 23 & 0 & 0 & 0 & 0 & 0 & 1 & 0 & 0 & 0 & 0 & 0 & 0 & 0 & 0 & 0 & 0 & 0 & 0 & 0 \\
\hline & 0 & 0 & 0 & 0 & 0 & 0 & 0 & 0 & 0 & 0 & 0 & 0 & 0 & & & & & & & \\
\hline$[3,3,0,0,0,0,1,1,0,0]$ & 19 & 0 & 0 & 0 & 8 & 0 & 1 & 0 & 0 & 0 & 0 & 0 & 0 & 0 & 0 & 0 & 0 & 0 & 0 & 0 \\
\hline & 0 & 0 & 0 & 0 & 0 & 0 & 0 & 0 & 0 & 0 & 0 & 0 & 0 & & & & & & & \\
\hline$[3,3,0,0,0,0,2,0,0,0]^{*}$ & $23 / 2$ & 0 & 0 & 0 & 0 & 0 & $1 / 2$ & 0 & 0 & 0 & 0 & 0 & 0 & 0 & 0 & 0 & 0 & 0 & 0 & 0 \\
\hline & 0 & 0 & 0 & 0 & 0 & 0 & 0 & 0 & 0 & 0 & 0 & 0 & 0 & & & & & & & \\
\hline$[3,2,2,0,0,0,1,0,0,0]^{*}$ & 35 & 0 & 0 & 0 & 0 & 0 & 0 & 0 & 0 & 0 & 0 & 0 & 0 & 0 & 0 & 0 & 0 & 0 & 0 & 0 \\
\hline & 0 & 0 & 0 & 0 & 0 & 0 & 0 & 0 & 0 & 0 & 0 & 0 & 0 & & & & & & & \\
\hline$[3,2,0,0,0,0,2,1,0,0]^{*}$ & 35 & 0 & 0 & 0 & 0 & 0 & 0 & 0 & 0 & 0 & 0 & 0 & 0 & 0 & 0 & 0 & 0 & 0 & 0 & 0 \\
\hline & 0 & 0 & 0 & 0 & 0 & 0 & 0 & 0 & 0 & 0 & 0 & 0 & 0 & & & & & & & \\
\hline$[3,2,1,0,0,0,1,1,0,0]$ & 62 & 0 & 0 & 0 & 16 & 0 & 0 & 0 & 0 & 0 & 0 & 0 & 0 & 0 & 0 & 0 & 0 & 0 & 0 & 0 \\
\hline & 0 & 0 & 0 & 0 & 0 & 0 & 0 & 0 & 0 & 0 & 0 & 0 & 0 & & & & & & & \\
\hline$[3,2,0,0,0,0,1,1,1,0]^{*}$ & 70 & 0 & 0 & 0 & 0 & 0 & 0 & 0 & 0 & 0 & 0 & 0 & 0 & 0 & 0 & 0 & 0 & 0 & 0 & 0 \\
\hline & 0 & 0 & 0 & 0 & 0 & 0 & 0 & 0 & 0 & 0 & 0 & 0 & 0 & & & & & & & \\
\hline$[3,1,1,1,1,1,0,0,0,0]$ & 140 & 0 & 0 & 0 & 0 & 0 & 0 & 0 & 0 & 0 & 0 & 0 & 0 & 0 & 0 & 0 & 0 & 0 & 0 & 0 \\
\hline & 0 & 0 & 0 & 0 & 0 & 0 & 0 & 0 & 0 & 0 & 0 & 0 & 0 & & & & & & & \\
\hline$[3,1,1,1,1,0,1,0,0,0]$ & 140 & 0 & 0 & 0 & 0 & 0 & 0 & 0 & 0 & 0 & 0 & 0 & 0 & 0 & 0 & 0 & 0 & 0 & 0 & 0 \\
\hline & 0 & 0 & 0 & 0 & 0 & 0 & 0 & 0 & 0 & 0 & 0 & 0 & 0 & & & & & & & \\
\hline$[3,1,1,1,0,0,1,1,0,0]$ & 128 & 0 & 0 & 0 & 24 & 0 & 0 & 0 & 0 & 0 & 0 & 0 & 0 & 0 & 0 & 0 & 0 & 0 & 0 & 0 \\
\hline & 0 & 0 & 0 & 0 & 0 & 0 & 0 & 0 & 0 & 0 & 0 & 0 & 0 & & & & & & & \\
\hline$[3,1,1,0,0,0,1,1,1,0]^{*}$ & 140 & 0 & 0 & 0 & 0 & 0 & 0 & 0 & 0 & 0 & 0 & 0 & 0 & 0 & 0 & 0 & 0 & 0 & 0 & 0 \\
\hline & 0 & 0 & 0 & 0 & 0 & 0 & 0 & 0 & 0 & 0 & 0 & 0 & 0 & & & & & & & \\
\hline$[3,1,0,0,0,0,1,1,1,1]$ & 136 & 0 & 0 & 0 & 8 & 0 & 0 & 0 & 0 & 0 & 0 & 0 & 0 & 0 & 0 & 0 & 0 & 0 & 0 & 0 \\
\hline & 0 & 0 & 0 & 0 & 0 & 0 & 0 & 0 & 0 & 0 & 0 & 0 & 0 & & & & & & & \\
\hline$[3,0,0,0,0,0,3,2,0,0]^{*}$ & $23 / 2$ & 0 & 0 & 0 & 0 & 0 & $1 / 2$ & 0 & 0 & 0 & 0 & 0 & 0 & 0 & 0 & 0 & 0 & 0 & 0 & 0 \\
\hline & 0 & 0 & 0 & 0 & 0 & 0 & 0 & 0 & 0 & 0 & 0 & 0 & 0 & & & & & & & \\
\hline$[3,0,0,0,0,0,3,1,1,0]^{*}$ & 23 & 0 & 0 & 0 & 0 & 0 & 1 & 0 & 0 & 0 & 0 & 0 & 0 & 0 & 0 & 0 & 0 & 0 & 0 & 0 \\
\hline & 0 & 0 & 0 & 0 & 0 & 0 & 0 & 0 & 0 & 0 & 0 & 0 & 0 & & & & & & & \\
\hline$[3,0,0,0,0,0,2,2,1,0]^{*}$ & 35 & 0 & 0 & 0 & 0 & 0 & 0 & 0 & 0 & 0 & 0 & 0 & 0 & 0 & 0 & 0 & 0 & 0 & 0 & 0 \\
\hline & 0 & 0 & 0 & 0 & 0 & 0 & 0 & 0 & 0 & 0 & 0 & 0 & 0 & & & & & & & \\
\hline$[3,0,0,0,0,0,2,1,1,1]^{*}$ & 70 & 0 & 0 & 0 & 0 & 0 & 0 & 0 & 0 & 0 & 0 & 0 & 0 & 0 & 0 & 0 & 0 & 0 & 0 & 0 \\
\hline & 0 & 0 & 0 & 0 & 0 & 0 & 0 & 0 & 0 & 0 & 0 & 0 & 0 & & & & & & & \\
\hline$[3,2,1,1,0,0,1,0,0,0]^{*}$ & 70 & 0 & 0 & 0 & 0 & 0 & 0 & 0 & 0 & 0 & 0 & 0 & 0 & 0 & 0 & 0 & 0 & 0 & 0 & 0 \\
\hline & 0 & 0 & 0 & 0 & 0 & 0 & 0 & 0 & 0 & 0 & 0 & 0 & 0 & & & & & & & \\
\hline
\end{tabular}


Table 3. Cubane Derivatives as 3D Structural Isomers Under the Point Group $\mathbf{O}_{h}$ (Part 3)

\begin{tabular}{|c|c|c|c|c|c|c|c|c|c|c|c|c|c|c|c|c|c|c|c|c|}
\hline parti & $\begin{array}{c}\mathbf{C}_{1} \\
\mathbf{C}_{4 v}\end{array}$ & $\mathrm{C}_{2}$ & $\mathrm{C}_{2}^{\prime}$ & $\begin{array}{l}\mathbf{C}_{s} \\
\mathbf{D}^{\prime}\end{array}$ & $\begin{array}{l}\mathbf{C}_{s}^{\prime} \\
\mathbf{D}_{2 h}\end{array}$ & $\begin{array}{c}\mathbf{C}_{i} \\
\mathbf{D}_{2 h}^{\prime}\end{array}$ & $\begin{array}{c}\mathrm{C}_{3} \\
\mathrm{~T}\end{array}$ & $\begin{array}{c}\mathbf{C}_{4} \\
\mathbf{D}_{3 d}\end{array}$ & $\begin{array}{c}\mathbf{S}_{4} \\
\mathbf{D}_{4 h}\end{array}$ & $\begin{array}{c}\mathrm{D}_{2} \\
\mathrm{O}\end{array}$ & $\begin{array}{l}\mathbf{D}_{2}^{\prime} \\
\mathbf{T}_{h}\end{array}$ & $\begin{array}{l}\mathbf{C}_{2 v} \\
\mathbf{T}_{d}\end{array}$ & $\begin{array}{l}\mathrm{C}_{2 v}^{\prime} \\
\mathrm{O}_{h}\end{array}$ & $\mathbf{C}_{2 v}^{\prime \prime}$ & $\mathrm{C}_{2 h}$ & $\mathbf{C}_{2 h}^{\prime}$ & $\mathbf{D}_{3}$ & $\mathrm{C}_{3 v}$ & $\mathrm{C}_{3 i}$ & $\mathbf{D}_{4}$ \\
\hline \multirow[t]{2}{*}[2,2,2,0,0,0,2,0,0,0]{$^{*}$} & 48 & 3 & 6 & 0 & 0 & 0 & 0 & 0 & 0 & 0 & 0 & 0 & 0 & 0 & 0 & 0 & 0 & 0 & 0 & 0 \\
\hline & 0 & 0 & 0 & 0 & 0 & 0 & 0 & 0 & 0 & 0 & 0 & 0 & 0 & & & & & & & \\
\hline \multirow[t]{2}{*}[2,2,0,0,0,0,2,2,0,0]{} & 43 & 3 & 4 & 5 & 4 & 1 & 0 & 0 & 0 & 0 & 0 & 0 & 0 & 2 & 0 & 2 & 0 & 0 & 0 & 0 \\
\hline & 0 & 0 & 0 & 0 & 0 & 0 & 0 & 0 & 0 & 0 & 0 & 0 & 0 & & & & & & & \\
\hline \multirow[t]{2}{*}[2,0,0,0,0,0,2,2,2,0]{$^{*}$} & 48 & 3 & 6 & 0 & 0 & 0 & 0 & 0 & 0 & 0 & 0 & 0 & 0 & 0 & 0 & 0 & 0 & 0 & 0 & 0 \\
\hline & 0 & 0 & 0 & 0 & 0 & 0 & 0 & 0 & 0 & 0 & 0 & 0 & 0 & & & & & & & \\
\hline \multirow[t]{2}{*}[2,2,2,1,0,0,1,0,0,0]{$^{*}$} & 105 & 0 & 0 & 0 & 0 & 0 & 0 & 0 & 0 & 0 & 0 & 0 & 0 & 0 & 0 & 0 & 0 & 0 & 0 & 0 \\
\hline & 0 & 0 & 0 & 0 & 0 & 0 & 0 & 0 & 0 & 0 & 0 & 0 & 0 & & & & & & & \\
\hline \multirow[t]{2}{*}[2,2,2,0,0,0,1,1,0,0]{} & 92 & 0 & 0 & 6 & 18 & 2 & 0 & 0 & 0 & 0 & 0 & 0 & 0 & 0 & 0 & 0 & 0 & 0 & 0 & 0 \\
\hline & 0 & 0 & 0 & 0 & 0 & 0 & 0 & 0 & 0 & 0 & 0 & 0 & 0 & & & & & & & \\
\hline \multirow[t]{2}{*}[2,2,1,1,1,0,1,0,0,0]{$^{*}$} & 210 & 0 & 0 & 0 & 0 & 0 & 0 & 0 & 0 & 0 & 0 & 0 & 0 & 0 & 0 & 0 & 0 & 0 & 0 & 0 \\
\hline & 0 & 0 & 0 & 0 & 0 & 0 & 0 & 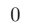 & 0 & 0 & 0 & 0 & 0 & & & & & & & \\
\hline \multirow[t]{2}{*}[2,2,1,1,0,0,1,1,0,0]{} & 198 & 0 & 0 & 0 & 24 & 0 & 0 & 0 & 0 & 0 & 0 & 0 & 0 & 0 & 0 & 0 & 0 & 0 & 0 & 0 \\
\hline & 0 & 0 & 0 & 0 & 0 & 0 & 0 & 0 & 0 & 0 & 0 & 0 & 0 & & & & & & & \\
\hline \multirow[t]{2}{*}[2,2,1,0,0,0,1,1,1,0]{$^{*}$} & 210 & 0 & 0 & 0 & 0 & 0 & 0 & 0 & 0 & 0 & 0 & 0 & 0 & 0 & 0 & 0 & 0 & 0 & 0 & 0 \\
\hline & 0 & 0 & 0 & 0 & 0 & 0 & 0 & 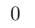 & 0 & 0 & 0 & 0 & 0 & & & & & & & \\
\hline \multirow[t]{2}{*}[2,2,0,0,0,0,1,1,1,1]{} & 196 & 0 & 0 & 12 & 12 & 4 & 0 & 0 & 0 & 0 & 0 & 0 & 0 & 0 & 0 & 0 & 0 & 0 & 0 & 0 \\
\hline & 0 & 0 & 0 & 0 & 0 & 0 & 0 & 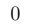 & 0 & 0 & 0 & 0 & 0 & & & & & & & \\
\hline \multirow[t]{2}{*}[2,1,1,1,1,1,1,0,0,0]{$^{*}$} & 420 & 0 & 0 & 0 & 0 & 0 & 0 & 0 & 0 & 0 & 0 & 0 & 0 & 0 & 0 & 0 & 0 & 0 & 0 & 0 \\
\hline & 0 & 0 & 0 & 0 & 0 & 0 & 0 & 0 & 0 & 0 & 0 & 0 & 0 & & & & & & & \\
\hline \multirow[t]{2}{*}[2,1,1,1,1,0,1,1,0,0]{} & 408 & 0 & 0 & 0 & 24 & 0 & 0 & 0 & 0 & 0 & 0 & 0 & 0 & 0 & 0 & 0 & 0 & 0 & 0 & 0 \\
\hline & 0 & 0 & 0 & 0 & 0 & 0 & 0 & 0 & 0 & 0 & 0 & 0 & 0 & & & & & & & \\
\hline \multirow[t]{2}{*}[2,1,1,1,0,0,1,1,1,0]{$^{*}$} & 420 & 0 & 0 & 0 & 0 & 0 & 0 & 0 & 0 & 0 & 0 & 0 & 0 & 0 & 0 & 0 & 0 & 0 & 0 & 0 \\
\hline & 0 & 0 & 0 & 0 & 0 & 0 & 0 & 0 & 0 & 0 & 0 & 0 & 0 & & & & & & & \\
\hline \multirow[t]{2}{*}[2,1,1,0,0,0,1,1,1,1]{} & 408 & 0 & 0 & 0 & 24 & 0 & 0 & 0 & 0 & 0 & 0 & 0 & 0 & 0 & 0 & 0 & 0 & 0 & 0 & 0 \\
\hline & 0 & 0 & 0 & 0 & 0 & 0 & 0 & 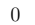 & 0 & 0 & 0 & 0 & 0 & & & & & & & \\
\hline \multirow[t]{2}{*}[1,1,1,1,1,1,2,0,0,0]{$^{*}$} & 420 & 0 & 0 & 0 & 0 & 0 & 0 & 0 & 0 & 0 & 0 & 0 & 0 & 0 & 0 & 0 & 0 & 0 & 0 & 0 \\
\hline & 0 & 0 & 0 & 0 & 0 & 0 & 0 & 0 & 0 & 0 & 0 & 0 & 0 & & & & & & & \\
\hline \multirow[t]{2}{*}[1,1,1,1,1,0,2,1,0,0]{$^{*}$} & 420 & 0 & 0 & 0 & 0 & 0 & 0 & 0 & 0 & 0 & 0 & 0 & 0 & 0 & 0 & 0 & 0 & 0 & 0 & 0 \\
\hline & 0 & 0 & 0 & 0 & 0 & 0 & 0 & 0 & 0 & 0 & 0 & 0 & 0 & & & & & & & \\
\hline \multirow[t]{2}{*}[1,1,1,1,0,0,2,1,1,0]{$^{*}$} & 420 & 0 & 0 & 0 & 0 & 0 & 0 & 0 & 0 & 0 & 0 & 0 & 0 & 0 & 0 & 0 & 0 & 0 & 0 & 0 \\
\hline & 0 & 0 & 0 & 0 & 0 & 0 & 0 & 0 & 0 & 0 & 0 & 0 & 0 & & & & & & & \\
\hline \multirow[t]{2}{*}[1,1,1,0,0,0,2,1,1,1]{$^{*}$} & 420 & 0 & 0 & 0 & 0 & 0 & 0 & 0 & 0 & 0 & 0 & 0 & 0 & 0 & 0 & 0 & 0 & 0 & 0 & 0 \\
\hline & 0 & 0 & 0 & 0 & 0 & 0 & 0 & 0 & 0 & 0 & 0 & 0 & 0 & & & & & & & \\
\hline \multirow[t]{2}{*}[1,1,1,1,1,1,1,1,0,0]{} & 840 & 0 & 0 & 0 & 0 & 0 & 0 & 0 & 0 & 0 & 0 & 0 & 0 & 0 & 0 & 0 & 0 & 0 & 0 & 0 \\
\hline & 0 & 0 & 0 & 0 & 0 & 0 & 0 & 0 & 0 & 0 & 0 & 0 & 0 & & & & & & & \\
\hline$[1,1,1,1,1,0,1,1,1,0]^{*}$ & 840 & 0 & 0 & 0 & 0 & 0 & 0 & 0 & 0 & 0 & 0 & 0 & 0 & 0 & 0 & 0 & 0 & 0 & 0 & 0 \\
\hline & 0 & 0 & 0 & 0 & 0 & 0 & 0 & 0 & 0 & 0 & 0 & 0 & 0 & & & & & & & \\
\hline$[1,1,1,1,0,0,1,1,1,1]$ & 816 & 0 & 0 & 0 & 48 & 0 & 0 & 0 & 0 & 0 & 0 & 0 & 0 & 0 & 0 & 0 & 0 & 0 & 0 & 0 \\
\hline & 0 & 0 & 0 & 0 & 0 & 0 & 0 & 0 & 0 & 0 & 0 & 0 & 0 & & & & & & & \\
\hline
\end{tabular}

\subsection{Symmetry-itermized enumeration under $R S$-stereogenic group $\mathrm{O}_{\tilde{\sigma}}$}

The PCI-CFs calculated under the $R S$-stereogenic group $\mathbf{O}_{\widetilde{\sigma}}$ (Eqs. 42-74) are used in symmetry-itemized enumeration of cubane derivatives under the $R S$-stereogenic group $\mathrm{O}_{\widetilde{\sigma}}$.

The same ligand inventory L (Eq. 109) is adopted. Because these PCI-CFs depend only on a hemispheric indices $b_{d}$, the ligand-inventory function shown by Eq. 111 is employed in the enumeration under $\mathbf{O}_{\tilde{\sigma}}$.

After the introduction of the ligand-inventory function (Eq. 111) is introduced into the PCI-CFs (Eqs. 42-74), the resulting polynomials are expanded into the respective generating functions, the coefficient of each term $\mathrm{H}^{h} \mathrm{~A}^{a} \mathrm{~B}^{b} \mathrm{X}^{x} \mathrm{Y}^{y} \mathrm{Z}^{z} \mathrm{p}^{p} \overline{\mathrm{p}}^{\bar{p}} \mathrm{q}^{q} \overline{\mathrm{q}}^{\bar{q}}$ is extracted to 
give Tables $4-6$.

\subsection{Symmetry-itermized enumeration under $L R$-permutation group $\mathrm{O}_{\widehat{I}}$}

As discussed in Subsection 3.3, the PCI-CFs under the LR-permutation group $\mathbf{O}_{\widehat{I}}$ are aligned by adopting the standard mark table and the USCI-CF table, which are obtained with no sorting applied to the native GAP mark table. The PCI-CFs calculated under the $L R$-permutation group $\mathbf{O}_{\widehat{I}}$ (Eqs. 76-108) are used in symmetry-itemized enumeration of cubane derivatives under the $L R$-permutation group $\mathbf{O}_{\widehat{I}}$ (Eq. 5).

The same ligand inventory L (Eq. 109) is adopted. Because the employed PCI-CFs depend on USCI-CFs with three kinds of sphericity indices $\left(\$_{d}: a_{d}, b_{d}\right.$, and $\left.c_{d}\right)$, the ligand-inventory functions shown by Eq. 110-112 are employed in the enumeration under $\mathrm{O}_{\widehat{I}}$.

After the ligand-inventory functions (Eqs. 110-112) are introduced into the PCI-CFs (Eqs. 42-74), the resulting polynomials are expanded into the respective generating functions. The coefficient of each term $\mathrm{H}^{h} \mathrm{~A}^{a} \mathrm{~B}^{b} \mathrm{X}^{x} \mathrm{Y}^{y} \mathrm{Z}^{z} \mathrm{p}^{p} \overline{\mathrm{p}}^{\bar{p}} \mathrm{q}^{q} \overline{\mathrm{q}}^{\bar{q}}$ is extracted to give Tables 7-9, which summarize the enumeration results based on the respective partitions $[h, a, b, x, y, z ; p, \bar{p}, q, \bar{q}]$ for the sake of convenience in enumeration.

The symmetrical appearance permits us to presume $h \geq a \geq w \geq x \geq y \geq z ; p \geq q$, $p \geq \bar{p}$, and $q \geq \bar{q}$ without losing generality. Appendix B shows a typical procedure for Fujita's USCI approach by using the combined-permutation representation (CPR). The coefficients obtained are collected in a tabular form (Tables 7-9).

\subsection{Five types of stereoisograms based on a cubane skeleton}

The above discussions deal with symmetry-itemized enumerations concerning three subgroups for specifying stereoisograms of cubane derivatives, i.e., the point group $\mathbf{O}_{h}$ (Eq. 2), the $R S$-stereogenic group $\mathbf{O}_{\widetilde{\sigma}}$ (Eq. 3), and the $L R$-permutation group $\mathbf{O}_{\widehat{I}}$ (Eq. 5). Thereby, the $R S$-stereoisomeric group $\mathbf{O}_{h \widetilde{\sigma} \widehat{I}}$ providing the integration of three aspects of stereoisograms $[8,9,11]$ can be discussed more detailedly in symmetry-itemized fashion. Thus, chirality/achirality can be discussed on the basis of enumeration data under $\mathbf{O}_{h}$; $R S$-stereogenicity/RS-astereogenicity can be discussed on the basis of enumeration data under $\mathbf{O}_{\widehat{I}}$, sclerality/asclerality can be discussed on the basis of enumeration data under $\mathbf{O}_{\widehat{I}}$. Such detailed enumeration results as based on the three subgroups provide us with 
Table 4. Cubane Derivatives as 3D Structural Isomers Under the $R S$-Stereogenic Group $\mathbf{O}_{\widetilde{\sigma}}$ (Part 1)

\begin{tabular}{|c|c|c|c|c|c|c|c|c|c|c|c|c|c|c|c|c|c|c|c|c|}
\hline part & $\begin{array}{c}\mathbf{C}_{1} \\
\widetilde{\mathbf{C}}_{4 v}\end{array}$ & $\mathrm{C}_{2}$ & $\begin{array}{l}\mathbf{C}_{2}^{\prime} \\
\widetilde{\mathbf{D}}_{2}\end{array}$ & $\begin{array}{l}\widetilde{\mathbf{C}}_{s} \\
\widetilde{\mathbf{D}}^{\prime}\end{array}$ & $\begin{array}{c}\widetilde{\mathbf{C}}_{s}^{\prime} \\
\widetilde{\mathbf{D}}\end{array}$ & $\begin{array}{r}\widetilde{\mathbf{C}}_{i} \\
\widetilde{\mathbf{D}}_{2}^{\prime}\end{array}$ & $\begin{array}{c}\mathrm{C}_{3} \\
\mathbf{T}\end{array}$ & $\begin{array}{c}\mathbf{C}_{4} \\
\widetilde{\mathbf{D}}_{3 d}\end{array}$ & $\begin{array}{c}\widetilde{\mathbf{S}}_{4} \\
\widetilde{\mathbf{D}}_{4 h}\end{array}$ & $\begin{array}{c}\mathrm{D}_{2} \\
\mathrm{O}\end{array}$ & $\begin{array}{l}\mathbf{D}_{2}^{\prime} \\
\widetilde{\mathbf{T}}_{h}\end{array}$ & $\begin{array}{l}\widetilde{\mathbf{C}}_{2 v} \\
\widetilde{\mathbf{T}}_{d}\end{array}$ & $\begin{array}{l}\widetilde{\mathbf{C}}_{2 v}^{\prime} \\
\mathrm{O}_{\widetilde{\sigma}}\end{array}$ & $\mathbf{C}_{2 v}^{\prime \prime}$ & $\mathrm{C}_{2 h}$ & $\widetilde{\mathbf{C}}_{2 h}^{\prime}$ & $\mathbf{D}_{3}$ & $\mathrm{C}_{3 v}$ & $\widetilde{\mathbf{C}}_{3 i}$ & $\mathrm{D}_{4}$ \\
\hline \multirow[t]{2}{*}[8,0,0,0,0,0,0,0,0,0]{} & 0 & 0 & 0 & 0 & 0 & 0 & 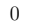 & 0 & 0 & 0 & 0 & 0 & 0 & 0 & 0 & 0 & 0 & 0 & 0 & 0 \\
\hline & 0 & 0 & 0 & 0 & 0 & 0 & 0 & c & 0 & 0 & 0 & 0 & 1 & & & & & & & \\
\hline \multirow[t]{2}{*}[7,1,0,0,0,0,0,0,0,0]{} & 0 & 0 & 0 & 0 & 0 & 0 & 0 & 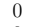 & 0 & 0 & 0 & 0 & 0 & 0 & 0 & 0 & 0 & 1 & 0 & 0 \\
\hline & 0 & 0 & 0 & 0 & 0 & 0 & 0 & 0 & 0 & 0 & 0 & 0 & 0 & & & & & & & \\
\hline \multirow[t]{2}{*}[6,2,0,0,0,0,0,0,0,0]{} & 0 & 0 & 0 & 0 & 0 & 0 & 0 & 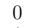 & 0 & 0 & 0 & 0 & 1 & 1 & 0 & 0 & 0 & 0 & 0 & 0 \\
\hline & 0 & 0 & 0 & 0 & 0 & 0 & 0 & 1 & 0 & 0 & 0 & 0 & 0 & & & & & & & \\
\hline \multirow[t]{2}{*}[6,1,1,0,0,0,0,0,0,0]{} & 0 & 0 & 0 & 0 & 2 & 0 & 0 & 0 & 0 & 0 & 0 & 0 & 0 & 0 & 0 & 0 & 0 & 1 & 0 & 0 \\
\hline & 0 & 0 & 0 & 0 & 0 & 0 & 0 & 0 & 0 & 0 & 0 & 0 & 0 & & & & & & & \\
\hline \multirow[t]{2}{*}[5,3,0,0,0,0,0,0,0,0]{} & 0 & 0 & 0 & 0 & 2 & 0 & 0 & 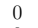 & 0 & 0 & 0 & 0 & 0 & 0 & 0 & 0 & 0 & 1 & 0 & 0 \\
\hline & 0 & 0 & 0 & 0 & 0 & 0 & 0 & 0 & 0 & 0 & 0 & 0 & 0 & & & & & & & \\
\hline \multirow[t]{2}{*}[5,2,1,0,0,0,0,0,0,0]{} & 1 & 0 & 0 & 0 & 5 & 0 & 0 & 0 & 0 & 0 & 0 & 0 & 0 & 0 & 0 & 0 & 0 & 0 & 0 & 0 \\
\hline & 0 & 0 & 0 & 0 & 0 & 0 & 0 & 0 & 0 & 0 & 0 & 0 & 0 & & & & & & & \\
\hline \multirow[t]{2}{*}[5,1,1,1,0,0,0,0,0,0]{} & 4 & 0 & 0 & 0 & 6 & 0 & 0 & 0 & 0 & 0 & 0 & 0 & 0 & 0 & 0 & 0 & 0 & 0 & 0 & 0 \\
\hline & 0 & 0 & 0 & 0 & 0 & 0 & 0 & 0 & 0 & 0 & 0 & 0 & 0 & & & & & & & \\
\hline \multirow[t]{2}{*}[4,4,0,0,0,0,0,0,0,0]{} & 0 & 0 & 1 & 0 & 1 & 0 & 0 & 0 & 0 & 0 & 0 & 0 & 0 & 0 & 0 & 0 & 0 & 1 & 0 & 0 \\
\hline & 1 & 0 & 0 & 0 & 0 & 1 & 0 & 0 & 0 & 0 & 0 & 1 & 0 & & & & & & & \\
\hline$[4,3,1,0,0,0,0,0,0,0]$ & 3 & 0 & 0 & 0 & 5 & 0 & 0 & 0 & 0 & 0 & 0 & 0 & 0 & 0 & 0 & 0 & 0 & 2 & 0 & 0 \\
\hline & 0 & 0 & 0 & 0 & 0 & 0 & 0 & 0 & 0 & 0 & 0 & 0 & 0 & & & & & & & \\
\hline$[4,2,2,0,0,0,0,0,0,0]$ & 4 & 0 & 2 & 1 & 4 & 0 & 0 & 0 & 0 & 0 & 0 & 0 & 3 & 1 & 0 & 1 & 0 & 0 & 0 & 0 \\
\hline & 0 & 0 & 0 & 0 & 0 & 0 & 0 & 0 & 0 & 0 & 0 & 0 & 0 & & & & & & & \\
\hline$[4,2,1,1,0,0,0,0,0,0]$ & 13 & 0 & 0 & 0 & 9 & 0 & . & 0 & 0 & 0 & 0 & 0 & 0 & 0 & 0 & 0 & 0 & 0 & 0 & 0 \\
\hline & 0 & 0 & 0 & 0 & 0 & 0 & 0 & c & 0 & 0 & 0 & 0 & 0 & & & & & & & \\
\hline$[4,1,1,1,1,0,0,0,0,0]$ & 32 & 0 & 0 & 0 & 6 & 0 & 0 & 0 & 0 & 0 & 0 & 0 & 0 & 0 & 0 & 0 & 0 & 0 & 0 & 0 \\
\hline & 0 & 0 & 0 & 0 & 0 & 0 & 0 & c & ) & 0 & 0 & 0 & ) & & & & & & & \\
\hline$[3,3,2,0,0,0,0,0,0,0]$ & 7 & 0 & 0 & 0 & 9 & 0 & 0 & 0 & 0 & 0 & 0 & 0 & 0 & 0 & 0 & 0 & 0 & 1 & 0 & 0 \\
\hline & 0 & 0 & 0 & 0 & 0 & 0 & 0 & 0 & 0 & 0 & 0 & 0 & 0 & & & & & & & \\
\hline$[3,3,1,1,0,0,0,0,0,0]$ & 18 & 0 & 0 & 0 & 10 & 0 & 0 & c & 0 & 0 & 0 & 0 & 0 & 0 & 0 & 0 & 0 & 2 & 0 & 0 \\
\hline & 0 & 0 & 0 & 0 & 0 & 0 & . & 0 & 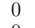 & 0 & 0 & 0 & 0 & & & & & & & \\
\hline$[3,2,2,1,0,0,0,0,0,0]$ & 28 & 0 & 0 & 0 & 14 & 0 & 0 & 0 & 0 & 0 & 0 & 0 & 0 & 0 & 0 & 0 & 0 & 0 & 0 & 0 \\
\hline & 0 & 0 & 0 & 0 & 0 & 0 & 0 & 0 & 0 & 0 & 0 & 0 & 0 & & & & & & & \\
\hline$[3,2,1,1,1,0,0,0,0,0]$ & 64 & 0 & 0 & 0 & 12 & 0 & 0 & 0 & 0 & 0 & 0 & 0 & 0 & 0 & 0 & 0 & 0 & 0 & 0 & 0 \\
\hline & 0 & 0 & 0 & 0 & 0 & 0 & 0 & 0 & 0 & 0 & 0 & 0 & 0 & & & & & & & \\
\hline$[2,2,2,2,0,0,0,0,0,0]$ & 40 & 0 & 6 & 3 & 12 & 1 & 0 & 0 & 0 & 0 & 0 & 0 & 6 & 0 & 0 & 0 & 0 & 0 & 0 & 0 \\
\hline & 0 & 0 & 0 & 0 & 0 & 0 & 0 & 0 & 0 & 0 & 0 & 0 & 0 & & & & & & & \\
\hline$[2,2,2,1,1,0,0,0,0,0]$ & 96 & 0 & 0 & 0 & 18 & 0 & 0 & 0 & 0 & 0 & 0 & 0 & 0 & 0 & 0 & 0 & 0 & 0 & 0 & 0 \\
\hline & 0 & 0 & 0 & 0 & 0 & 0 & 0 & & 0 & 0 & 0 & 0 & ) & & & & & & & \\
\hline$[2,2,1,1,1,1,0,0,0,0]$ & 204 & 0 & 0 & 0 & 12 & 0 & 0 & 0 & 0 & 0 & 0 & 0 & 0 & 0 & 0 & 0 & 0 & 0 & 0 & 0 \\
\hline & 0 & 0 & 0 & 0 & 0 & 0 & 0 & & 0 & 0 & 0 & 0 & 0 & & & & & & & \\
\hline$[7,0,0,0,0,0,1,0,0,0]^{*}$ & 0 & 0 & 0 & 0 & 0 & 0 & 0 & 0 & 0 & 0 & 0 & 0 & 0 & 0 & 0 & 0 & 0 & 1 & 0 & 0 \\
\hline & 0 & 0 & 0 & 0 & 0 & 0 & 0 & 0 & 0 & 0 & 0 & 0 & 0 & & & & & & & \\
\hline$[6,1,0,0,0,0,1,0,0,0]^{*}$ & 0 & 0 & 0 & 0 & 2 & 0 & 0 & 0 & 0 & 0 & 0 & 0 & 0 & 0 & 0 & 0 & 0 & 1 & 0 & 0 \\
\hline & 0 & 0 & 0 & 0 & 0 & 0 & 0 & 0 & 0 & 0 & 0 & 0 & 0 & & & & & & & \\
\hline$[6,0,0,0,0,0,2,0,0,0]^{*}$ & 0 & 0 & 0 & 0 & 0 & 0 & 0 & 0 & 0 & 0 & 0 & 0 & 1 & 1 & 0 & 0 & 0 & 0 & 0 & 0 \\
\hline & 0 & 0 & 0 & 0 & 0 & 0 & 0 & 1 & 0 & 0 & 0 & 0 & 0 & & & & & & & \\
\hline$[6,0,0,0,0,0,1,1,0,0]$ & 0 & 0 & 0 & 0 & 2 & 0 & 0 & 0 & 0 & 0 & 0 & 0 & 0 & 0 & 0 & 0 & 0 & 1 & 0 & 0 \\
\hline & 0 & 0 & 0 & 0 & 0 & 0 & 0 & 0 & 0 & 0 & 0 & 0 & 0 & & & & & & & \\
\hline$[5,2,0,0,0,0,1,0,0,0]^{*}$ & 1 & 0 & 0 & 0 & 5 & 0 & 0 & 0 & 0 & 0 & 0 & 0 & 0 & 0 & 0 & 0 & 0 & 0 & 0 & 0 \\
\hline & 0 & 0 & 0 & 0 & 0 & 0 & 0 & & . & 0 & 0 & 0 & 0 & & & & & & & \\
\hline$[5,1,1,0,0,0,1,0,0,0]^{*}$ & 4 & 0 & 0 & 0 & 6 & 0 & 0 & 0 & 0 & 0 & 0 & 0 & 0 & 0 & 0 & 0 & 0 & 0 & 0 & 0 \\
\hline & 0 & 0 & 0 & 0 & 0 & 0 & 0 & 0 & 0 & 0 & 0 & 0 & 0 & & & & & & & \\
\hline$[5,1,0,0,0,0,2,0,0,0]^{*}$ & 1 & 0 & 0 & 0 & 5 & 0 & 0 & 0 & 0 & 0 & 0 & 0 & 0 & 0 & 0 & 0 & 0 & 0 & 0 & 0 \\
\hline & 0 & 0 & 0 & 0 & 0 & 0 & 0 & 0 & 0 & 0 & 0 & 0 & 0 & & & & & & & \\
\hline$[5,1,0,0,0,0,1,1,0,0]$ & 4 & 0 & 0 & 0 & 6 & 0 & 0 & 0 & 0 & 0 & 0 & 0 & 0 & 0 & 0 & 0 & 0 & 0 & 0 & 0 \\
\hline & 0 & 0 & 0 & 0 & 0 & 0 & 0 & 0 & 0 & 0 & 0 & 0 & 0 & & & & & & & \\
\hline$[5,0,0,0,0,0,3,0,0,0]^{*}$ & 0 & 0 & 0 & 0 & 2 & 0 & 0 & 0 & 0 & 0 & 0 & 0 & 0 & 0 & 0 & 0 & 0 & 1 & 0 & 0 \\
\hline & 0 & 0 & 0 & 0 & 0 & 0 & 0 & 0 & 0 & 0 & 0 & 0 & 0 & & & & & & & \\
\hline$[5,0,0,0,0,0,2,1,0,0]^{*}$ & 1 & 0 & 0 & 0 & 5 & 0 & 0 & 0 & 0 & 0 & 0 & 0 & 0 & 0 & 0 & 0 & 0 & 0 & 0 & 0 \\
\hline & 0 & 0 & 0 & 0 & 0 & 0 & 0 & 0 & 0 & 0 & 0 & 0 & 0 & & & & & & & \\
\hline$[5,0,0,0,0,0,1,1,1,0]^{*}$ & 4 & 0 & 0 & 0 & 6 & 0 & 0 & 0 & 0 & 0 & 0 & 0 & 0 & 0 & 0 & 0 & 0 & 0 & 0 & 0 \\
\hline & 0 & 0 & 0 & 0 & 0 & 0 & 0 & 0 & 0 & 0 & 0 & 0 & 0 & & & & & & & \\
\hline
\end{tabular}


Table 5. Cubane Derivatives as 3D Structural Isomers Under the $R S$-Stereogenic Group $\mathbf{O}_{\tilde{\sigma}}$ (Part 2)

\begin{tabular}{|c|c|c|c|c|c|c|c|c|c|c|c|c|c|c|c|c|c|c|c|c|}
\hline partition & $\begin{array}{c}\mathbf{C}_{1} \\
\widetilde{\mathbf{C}}_{4 v}\end{array}$ & $\begin{array}{c}\mathbf{C}_{2} \\
\widetilde{\mathbf{C}}_{4 h}\end{array}$ & $\begin{array}{l}\mathrm{C}_{2}^{\prime} \\
\widetilde{\mathrm{D}}_{2}\end{array}$ & $\begin{array}{c}\widetilde{\mathbf{C}}_{s} \\
\widetilde{\mathbf{D}}^{\prime}\end{array}$ & $\begin{array}{l}\widetilde{\mathbf{C}}_{s}^{\prime} \\
\widetilde{\mathbf{D}}\end{array}$ & $\begin{array}{c}\widetilde{\mathbf{C}}_{i} \\
\widetilde{\mathbf{D}}_{2 h}^{\prime}\end{array}$ & $\begin{array}{c}\mathbf{C}_{3} \\
\mathbf{T}\end{array}$ & $\begin{array}{c}\mathbf{C}_{4} \\
\widetilde{\mathbf{D}}_{3 d}\end{array}$ & $\begin{array}{c}\widetilde{\mathbf{S}}_{4} \\
\widetilde{\mathbf{D}}_{4 h}\end{array}$ & $\begin{array}{c}\mathrm{D}_{2} \\
\mathrm{O}\end{array}$ & $\mathbf{T}_{h}$ & $\begin{array}{l}\mathbf{C}_{2 v} \\
\widetilde{\mathbf{T}}_{d}\end{array}$ & $\begin{array}{l}\mathbf{C}_{2 v}^{\prime} \\
\mathbf{O}_{\widetilde{\sigma}}\end{array}$ & $\mathbf{C}_{2 v}^{\prime \prime}$ & $\mathrm{C}_{2 h}$ & $\widetilde{\mathbf{C}}_{2 h}^{\prime}$ & $\mathbf{D}_{3}$ & $\widetilde{\mathbf{C}}_{3 v}$ & $\widetilde{\mathbf{C}}_{3 i}$ & $\mathrm{D}_{4}$ \\
\hline \multirow[t]{2}{*}[4,0,0,0,0,0,4,0,0,0]{$^{*}$} & 0 & 0 & 1 & 0 & 1 & 0 & 0 & 0 & 0 & 0 & 0 & 0 & 0 & 0 & 0 & 0 & 0 & 1 & 0 & 0 \\
\hline & 1 & 0 & 0 & 0 & 0 & 1 & 0 & 0 & 0 & 0 & 0 & 1 & 0 & & & & & & & \\
\hline \multirow[t]{2}{*}[4,3,0,0,0,0,1,0,0,0]{$^{*}$} & 3 & 0 & 0 & 0 & 5 & 0 & 0 & 0 & 0 & 0 & 0 & 0 & 0 & 0 & 0 & 0 & 0 & 2 & 0 & 0 \\
\hline & 0 & 0 & 0 & 0 & 0 & 0 & 0 & 0 & 0 & 0 & 0 & 0 & 0 & & & & & & & \\
\hline \multirow[t]{2}{*}[4,2,0,0,0,0,2,0,0,0]{$^{*}$} & 4 & 0 & 2 & 1 & 4 & 0 & 0 & 0 & 0 & 0 & 0 & 0 & 3 & 1 & 0 & 1 & 0 & 0 & 0 & 0 \\
\hline & 0 & 0 & 0 & 0 & 0 & 0 & 0 & 0 & 0 & 0 & 0 & 0 & 0 & & & & & & & \\
\hline \multirow{2}{*}[4,0,0,0,0,0,2,2,0,0]{} & 4 & 0 & 2 & 1 & 4 & 0 & 0 & 0 & 0 & 0 & 0 & 0 & 3 & 1 & 0 & 1 & 0 & 0 & 0 & 0 \\
\hline & 0 & 0 & 0 & 0 & 0 & 0 & 0 & 0 & 0 & 0 & 0 & 0 & 0 & & & & & & & \\
\hline \multirow[t]{2}{*}[4,0,0,0,0,0,2,0,2,0]{$^{*}$} & 4 & 0 & 2 & 1 & 4 & 0 & 0 & 0 & 0 & 0 & 0 & 0 & 3 & 1 & 0 & 1 & 0 & 0 & 0 & 0 \\
\hline & 0 & 0 & 0 & 0 & 0 & 0 & 0 & 0 & 0 & 0 & 0 & 0 & 0 & & & & & & & \\
\hline \multirow[t]{2}{*}[4,2,1,0,0,0,1,0,0,0]{$^{*}$} & 13 & 0 & 0 & 0 & 9 & 0 & 0 & 0 & 0 & 0 & 0 & 0 & 0 & 0 & 0 & 0 & 0 & 0 & 0 & 0 \\
\hline & 0 & 0 & 0 & 0 & 0 & 0 & 0 & 0 & 0 & 0 & 0 & 0 & 0 & & & & & & & \\
\hline \multirow[t]{2}{*}[4,2,0,0,0,0,1,1,0,0]{} & 13 & 0 & 0 & 0 & 9 & 0 & 0 & 0 & 0 & 0 & 0 & 0 & 0 & 0 & 0 & 0 & 0 & 0 & 0 & 0 \\
\hline & 0 & 0 & 0 & 0 & 0 & 0 & 0 & 0 & 0 & 0 & 0 & 0 & 0 & & & & & & & \\
\hline \multirow[t]{2}{*}[4,1,1,1,0,0,1,0,0,0]{$^{*}$} & 32 & 0 & 0 & 0 & 6 & 0 & 0 & 0 & 0 & 0 & 0 & 0 & 0 & 0 & 0 & 0 & 0 & 0 & 0 & 0 \\
\hline & 0 & 0 & 0 & 0 & 0 & 0 & 0 & 0 & 0 & 0 & 0 & 0 & 0 & & & & & & & \\
\hline \multirow{2}{*}[4,1,1,0,0,0,1,1,0,0]{} & 32 & 0 & 0 & 0 & 6 & 0 & 0 & 0 & 0 & 0 & 0 & 0 & 0 & 0 & 0 & 0 & 0 & 0 & 0 & 0 \\
\hline & 0 & 0 & 0 & 0 & 0 & 0 & 0 & 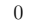 & 0 & 0 & 0 & 0 & 0 & & & & & & & \\
\hline \multirow[t]{2}{*}[4,1,0,0,0,0,1,1,1,0]{$^{*}$} & 32 & 0 & 0 & 0 & 6 & 0 & 0 & 0 & 0 & 0 & 0 & 0 & 0 & 0 & 0 & 0 & 0 & 0 & 0 & 0 \\
\hline & 0 & 0 & 0 & 0 & 0 & 0 & 0 & 0 & 0 & 0 & 0 & 0 & 0 & & & & & & & \\
\hline$[4,0,0,0,0,0,1,1,1,1]$ & 32 & 0 & 0 & 0 & 6 & 0 & 0 & 0 & 0 & 0 & 0 & 0 & 0 & 0 & 0 & 0 & 0 & 0 & 0 & 0 \\
\hline & 0 & 0 & 0 & 0 & 0 & 0 & 0 & 0 & 0 & 0 & 0 & 0 & 0 & & & & & & & \\
\hline$[3,3,1,0,0,0,1,0,0,0]^{*}$ & 18 & 0 & 0 & 0 & 10 & 0 & 0 & 0 & 0 & 0 & 0 & 0 & 0 & 0 & 0 & 0 & 0 & 2 & 0 & 0 \\
\hline & 0 & 0 & 0 & 0 & 0 & 0 & 0 & 0 & 0 & 0 & 0 & 0 & 0 & & & & & & & \\
\hline$[3,3,0,0,0,0,1,1,0,0]$ & 18 & 0 & 0 & 0 & 10 & 0 & 0 & 0 & 0 & 0 & 0 & 0 & 0 & 0 & 0 & 0 & 0 & 2 & 0 & 0 \\
\hline & 0 & 0 & 0 & 0 & 0 & 0 & 0 & 0 & 0 & 0 & 0 & 0 & 0 & & & & & & & \\
\hline$[3,3,0,0,0,0,2,0,0,0]^{*}$ & 7 & 0 & 0 & 0 & 9 & 0 & 0 & 0 & 0 & 0 & 0 & 0 & 0 & 0 & 0 & 0 & 0 & 1 & 0 & 0 \\
\hline & 0 & 0 & 0 & 0 & 0 & 0 & 0 & 0 & 0 & 0 & 0 & 0 & 0 & & & & & & & \\
\hline$[3,2,2,0,0,0,1,0,0,0]^{*}$ & 28 & 0 & 0 & 0 & 14 & 0 & 0 & 0 & 0 & 0 & 0 & 0 & 0 & 0 & 0 & 0 & 0 & 0 & 0 & 0 \\
\hline & 0 & 0 & 0 & 0 & 0 & 0 & 0 & 0 & 0 & 0 & 0 & 0 & 0 & & & & & & & \\
\hline$[3,2,0,0,0,0,2,1,0,0]^{*}$ & 28 & 0 & 0 & 0 & 14 & 0 & 0 & 0 & 0 & 0 & 0 & 0 & 0 & 0 & 0 & 0 & 0 & 0 & 0 & 0 \\
\hline & 0 & 0 & 0 & 0 & 0 & 0 & 0 & 0 & 0 & 0 & 0 & 0 & 0 & & & & & & & \\
\hline$[3,2,1,0,0,0,1,1,0,0]$ & 64 & 0 & 0 & 0 & 12 & 0 & 0 & 0 & 0 & 0 & 0 & 0 & 0 & 0 & 0 & 0 & 0 & 0 & 0 & 0 \\
\hline & 0 & 0 & 0 & 0 & 0 & 0 & 0 & 0 & 0 & 0 & 0 & 0 & 0 & & & & & & & \\
\hline$[3,2,0,0,0,0,1,1,1,0]^{*}$ & 64 & 0 & 0 & 0 & 12 & 0 & 0 & 0 & 0 & 0 & 0 & 0 & 0 & 0 & 0 & 0 & 0 & 0 & 0 & 0 \\
\hline & 0 & 0 & 0 & 0 & 0 & 0 & 0 & 0 & 0 & 0 & 0 & 0 & 0 & & & & & & & \\
\hline$[3,1,1,1,1,1,0,0,0,0]$ & 140 & 0 & 0 & 0 & 0 & 0 & 0 & 0 & 0 & 0 & 0 & 0 & 0 & 0 & 0 & 0 & 0 & 0 & 0 & 0 \\
\hline & 0 & 0 & 0 & 0 & 0 & 0 & 0 & 0 & 0 & 0 & 0 & 0 & 0 & & & & & & & \\
\hline$[3,1,1,1,1,0,1,0,0,0]^{*}$ & 140 & 0 & 0 & 0 & 0 & 0 & 0 & 0 & 0 & 0 & 0 & 0 & 0 & 0 & 0 & 0 & 0 & 0 & 0 & 0 \\
\hline & 0 & 0 & 0 & 0 & 0 & 0 & 0 & 0 & 0 & 0 & 0 & 0 & 0 & & & & & & & \\
\hline$[3,1,1,1,0,0,1,1,0,0]$ & 140 & 0 & 0 & 0 & 0 & 0 & 0 & 0 & 0 & 0 & 0 & 0 & 0 & 0 & 0 & 0 & 0 & 0 & 0 & 0 \\
\hline & 0 & 0 & 0 & 0 & 0 & 0 & 0 & 0 & 0 & 0 & 0 & 0 & 0 & & & & & & & \\
\hline$[3,1,1,0,0,0,1,1,1,0]^{*}$ & 140 & 0 & 0 & 0 & 0 & 0 & 0 & 0 & 0 & 0 & 0 & 0 & 0 & 0 & 0 & 0 & 0 & 0 & 0 & 0 \\
\hline & 0 & 0 & 0 & 0 & 0 & 0 & 0 & 0 & 0 & 0 & 0 & 0 & 0 & & & & & & & \\
\hline$[3,1,0,0,0,0,1,1,1,1]$ & 140 & 0 & 0 & 0 & 0 & 0 & 0 & 0 & 0 & 0 & 0 & 0 & 0 & 0 & 0 & 0 & 0 & 0 & 0 & 0 \\
\hline & 0 & 0 & 0 & 0 & 0 & 0 & 0 & 0 & 0 & 0 & 0 & 0 & 0 & & & & & & & \\
\hline$[3,0,0,0,0,0,3,2,0,0]^{*}$ & 7 & 0 & 0 & 0 & 9 & 0 & 0 & 0 & 0 & 0 & 0 & 0 & 0 & 0 & 0 & 0 & 0 & 1 & 0 & 0 \\
\hline & 0 & 0 & 0 & 0 & 0 & 0 & 0 & 0 & 0 & 0 & 0 & 0 & 0 & & & & & & & \\
\hline$[3,0,0,0,0,0,3,1,1,0]^{*}$ & 18 & 0 & 0 & 0 & 10 & 0 & 0 & 0 & 0 & 0 & 0 & 0 & 0 & 0 & 0 & 0 & 0 & 2 & 0 & 0 \\
\hline & 0 & 0 & 0 & 0 & 0 & 0 & 0 & 0 & 0 & 0 & 0 & 0 & 0 & & & & & & & \\
\hline$[3,0,0,0,0,0,2,2,1,0]^{*}$ & 28 & 0 & 0 & 0 & 14 & 0 & 0 & 0 & 0 & 0 & 0 & 0 & 0 & 0 & 0 & 0 & 0 & 0 & 0 & 0 \\
\hline & 0 & 0 & 0 & 0 & 0 & 0 & 0 & 0 & 0 & 0 & 0 & 0 & 0 & & & & & & & \\
\hline$[3,0,0,0,0,0,2,1,1,1]^{*}$ & 64 & 0 & 0 & 0 & 12 & 0 & 0 & 0 & 0 & 0 & 0 & 0 & 0 & 0 & 0 & 0 & 0 & 0 & 0 & 0 \\
\hline & 0 & 0 & 0 & 0 & 0 & 0 & 0 & 0 & 0 & 0 & 0 & 0 & 0 & & & & & & & \\
\hline$[3,2,1,1,0,0,1,0,0,0]^{*}$ & 64 & 0 & 0 & 0 & 12 & 0 & 0 & 0 & 0 & 0 & 0 & 0 & 0 & 0 & 0 & 0 & 0 & 0 & 0 & 0 \\
\hline & 0 & 0 & 0 & 0 & 0 & 0 & 0 & 0 & 0 & 0 & 0 & 0 & 0 & & & & & & & \\
\hline
\end{tabular}


Table 6. Cubane Derivatives as 3D Structural Isomers Under the $R S$-Stereogenic Group $\mathbf{O}_{\widetilde{\sigma}}$ (Part 3)

\begin{tabular}{|c|c|c|c|c|c|c|c|c|c|c|c|c|c|c|c|c|c|c|c|c|}
\hline partiti & $\begin{array}{c}\mathbf{C}_{1} \\
\widetilde{\mathbf{C}}_{4 v}\end{array}$ & & & $\mathbf{C}_{s}$ & & $\widetilde{\mathbf{D}}_{2 h}^{\prime}$ & $\mathrm{C}_{3}$ & & & $\mathbf{D}_{2}$ & $\widetilde{\mathbf{T}}_{h}$ & $\widetilde{\mathbf{T}}_{d}$ & $\mathrm{O}_{\widetilde{\sigma}}$ & & & & & C & & $\mathbf{D}_{4}$ \\
\hline \multirow[t]{2}{*}{$\overline{[2,2,2,0,0,0,2,0,0,0]^{*}}$} & 40 & 0 & 6 & 3 & 12 & 1 & 0 & 0 & 0 & 0 & 0 & 0 & 6 & 0 & 0 & 0 & 0 & 0 & 0 & 0 \\
\hline & 0 & 0 & 0 & 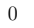 & 0 & 0 & 0 & 0 & 0 & 0 & 0 & 0 & 0 & & & & & & & \\
\hline \multirow[t]{2}{*}[2,2,0,0,0,0,2,2,0,0]{} & 40 & 0 & 6 & 3 & 12 & 1 & 0 & 0 & 0 & 0 & 0 & 0 & 0 & 0 & 0 & 0 & 0 & 0 & 0 & 0 \\
\hline & 0 & 0 & 0 & 0 & 0 & 0 & 0 & 0 & 0 & 0 & 0 & 0 & 0 & & & & & & & \\
\hline \multirow[t]{2}{*}[2,0,0,0,0,0,2,2,2,0]{$^{*}$} & 40 & 0 & s & 3 & 12 & 1 & 0 & 0 & 0 & 0 & 0 & 0 & 6 & 0 & 0 & 0 & 0 & 0 & 0 & 0 \\
\hline & 0 & 0 & 0 & 0 & 0 & 0 & 0 & 0 & 0 & 0 & 0 & 0 & 0 & & & & & & & \\
\hline \multirow[t]{2}{*}[2,2,2,1,0,0,1,0,0,0]{$^{*}$} & 96 & 0 & 0 & 0 & 18 & 0 & 0 & 0 & 0 & 0 & 0 & 0 & 0 & 0 & 0 & 0 & 0 & 0 & 0 & 0 \\
\hline & 0 & 0 & 0 & 0 & 0 & 0 & 0 & 0 & 0 & 0 & 0 & 0 & 0 & & & & & & & \\
\hline \multirow[t]{2}{*}[2,2,2,0,0,0,1,1,0,0]{} & 96 & 0 & 0 & 0 & 18 & 0 & 0 & 0 & 0 & 0 & 0 & 0 & 0 & 0 & 0 & 0 & 0 & 0 & 0 & 0 \\
\hline & 0 & 0 & 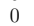 & 0 & 0 & 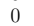 & & o & 0 & 0 & 0 & 0 & 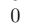 & & & & & & & \\
\hline \multirow[t]{2}{*}[2,2,1,1,1,0,1,0,0,0]{$^{*}$} & 204 & 0 & 0 & 0 & 12 & 0 & & 0 & 0 & 0 & 0 & & & 0 & 0 & 0 & 0 & 0 & 0 & 0 \\
\hline & 0 & 0 & 0 & 0 & 0 & 0 & 0 & 0 & 0 & 0 & 0 & 0 & 0 & & & & & & & \\
\hline \multirow[t]{2}{*}[2,2,1,1,0,0,1,1,0,0]{} & 204 & 0 & 0 & 0 & 12 & 0 & 0 & 0 & 0 & 0 & 0 & 0 & 0 & 0 & 0 & 0 & 0 & 0 & 0 & 0 \\
\hline & 0 & 0 & 0 & P & 0 & 0 & & 0 & 0 & 0 & 0 & & & & & & & & & \\
\hline \multirow[t]{2}{*}[2,2,1,0,0,0,1,1,1,0]{$^{*}$} & 204 & 0 & 0 & 0 & 12 & 0 & 0 & 0 & 0 & 0 & 0 & 0 & 0 & 0 & 0 & 0 & 0 & 0 & 0 & 0 \\
\hline & 0 & 0 & 8 & 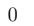 & 0 & 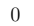 & o & 0 & 0 & 0 & 0 & 0 & 0 & & & & & & & \\
\hline \multirow[t]{2}{*}[2,2,0,0,0,0,1,1,1,1]{} & 204 & 0 & 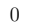 & 0 & 12 & 0 & & 0 & 0 & 0 & . & & & 0 & 0 & 0 & 0 & 0 & 0 & 0 \\
\hline & 0 & 0 & 0 & 0 & 0 & 0 & 0 & 0 & 0 & 0 & 0 & 0 & 0 & & & & & & & \\
\hline \multirow{2}{*}[2,1,1,1,1,1,1,0,0,0]{$^{*}$} & 420 & 0 & 两 & 0 & 0 & 0 & 0 & 0 & 0 & 0 & 0 & 0 & 0 & 0 & 0 & 0 & 0 & 0 & 0 & 0 \\
\hline & 0 & 0 & 0 & 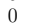 & 0 & 0 & 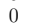 & 0 & 0 & 0 & 0 & 0 & 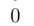 & & & & & & & \\
\hline \multirow[t]{2}{*}[2,1,1,1,1,0,1,1,0,0]{} & 420 & 0 & 0 & 0 & 0 & 0 & 0 & 0 & 0 & 0 & 0 & 0 & 0 & 0 & 0 & 0 & 0 & 0 & 0 & 0 \\
\hline & 0 & 0 & 0 & 0 & 0 & 0 & 0 & 0 & 0 & 0 & 0 & & & & & & & & & \\
\hline \multirow[t]{2}{*}[2,1,1,1,0,0,1,1,1,0]{$^{*}$} & 420 & 0 & 0 & 0 & 0 & 0 & 0 & 0 & 0 & 0 & 0 & 0 & 0 & 0 & 0 & 0 & 0 & 0 & 0 & 0 \\
\hline & 0 & 0 & 0 & 0 & 0 & 0 & 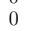 & 0 & 0 & 0 & 0 & 0 & 0 & & & & & & & \\
\hline \multirow[t]{2}{*}[2,1,1,0,0,0,1,1,1,1]{} & 420 & 0 & 0 & 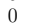 & 0 & 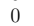 & 0 & 0 & 0 & 0 & 0 & 0 & 0 & 0 & 0 & 0 & 0 & 0 & 0 & 0 \\
\hline & 0 & 0 & 0 & 0 & 0 & 0 & 0 & 0 & 0 & 0 & 0 & 0 & 0 & & & & & & & \\
\hline \multirow{2}{*}[1,1,1,1,1,1,2,0,0,0]{$^{*}$} & 420 & 0 & 0 & 0 & 0 & 0 & 0 & 0 & 0 & 0 & 0 & 0 & 0 & 0 & 0 & 0 & 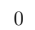 & 0 & 0 & 0 \\
\hline & 0 & 0 & 0 & 0 & 0 & 0 & & . & 0 & 0 & 0 & & & & & & & & & \\
\hline \multirow{2}{*}[1,1,1,1,1,0,2,1,0,0]{$^{*}$} & 420 & 0 & 0 & 0 & 0 & 0 & 0 & 0 & 0 & 0 & 0 & 0 & 0 & 0 & 0 & 0 & U & 0 & 0 & 0 \\
\hline & 0 & 0 & 0 & 0 & 0 & 0 & 0 & 0 & 0 & 0 & 0 & 0 & 0 & & & & & & & \\
\hline \multirow[t]{2}{*}[1,1,1,1,0,0,2,1,1,0]{$^{*}$} & 420 & 0 & 0 & 0 & 0 & 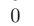 & 0 & . & 0 & 0 & 0 & 0 & 0 & 0 & 0 & 0 & 0 & 0 & 0 & 0 \\
\hline & 0 & 0 & 0 & 0 & 0 & 0 & 0 & 0 & 0 & 0 & 0 & 0 & 0 & & & & & & & \\
\hline \multirow[t]{2}{*}[1,1,1,0,0,0,2,1,1,1]{$^{*}$} & 420 & 0 & 0 & 0 & 0 & 0 & 0 & 0 & 0 & 0 & 0 & 0 & 0 & 0 & 0 & 0 & 0 & 0 & 0 & 0 \\
\hline & 0 & 0 & 0 & 0 & 0 & 0 & 0 & 0 & 0 & 0 & & & & & & & & & & \\
\hline \multirow[t]{2}{*}[1,1,1,1,1,1,1,1,0,0]{} & 840 & 0 & 0 & 0 & 0 & 0 & U & 0 & 0 & 0 & 0 & 0 & 0 & 0 & 0 & 0 & 0 & 0 & 0 & 0 \\
\hline & 0 & 0 & 0 & 0 & 0 & 0 & 0 & 0 & 0 & 0 & 0 & 0 & 0 & & & & & & & \\
\hline$[1,1,1,1,1,0,1,1,1,0]^{*}$ & 840 & 0 & 0 & 0 & 0 & 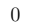 & 0 & 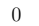 & 0 & 0 & 0 & 0 & 0 & 0 & 0 & 0 & 0 & 0 & U & 0 \\
\hline & 0 & 0 & 0 & 0 & 0 & 0 & 0 & 0 & 0 & 0 & 0 & 0 & & & & & & & & \\
\hline$[1,1,1,1,0,0,1,1,1,1]$ & 840 & 0 & 0 & 0 & 0 & 0 & 0 & 0 & 0 & 0 & 0 & 0 & 0 & 0 & 0 & 0 & 0 & 0 & 0 & 0 \\
\hline & 0 & U & 0 & 0 & 0 & 0 & 0 & 0 & 0 & 0 & 0 & 0 & 0 & & & & & & & \\
\hline
\end{tabular}

versatile methodology for clarifying the action of the $R S$-stereoisomeric group $\mathbf{O}_{h \widetilde{\sigma} \widehat{I}}$ on stereoisograms (Eq. 6 in Subsection 2.6) in symmetry-itemized fashion.

Under the action of an $R S$-stereoisomeric group $\mathbf{O}_{h \widetilde{\sigma} \widehat{I}}$, the three pairs of attributes in the corresponding stereoisogram, i.e., chirality/achirality (Subsection 4.1) along the vertical direction, $R S$-astereogenicity/RS-stereogenicity (Subsection 4.2) along the horizontal direction, and sclerality/asclerality (Subsection 4.3) along the diagonal direction, are combined on the basis of the elementary stereoisogram (Figure 1 in Subsection 2.1), so as to give five types of stereoisograms, as shown in Figure 5.

For our deeper information, it is useful to reveal the modification history of the charts of stereoisograms. At first, a primitive chart of stereoisograms has been reported as Figure 6 of the proposal of the concept of stereoisograms [8]. This primitive chart has 
Table 7. Cubane Derivatives as 3D Structural Isomers Under the LR-Permutation Group $\mathbf{O}_{\widehat{I}}$ (Part 1)

\begin{tabular}{|c|c|c|c|c|c|c|c|c|c|c|c|c|c|c|c|c|c|c|c|c|}
\hline partition & $\begin{array}{l}\mathbf{C}_{1} \\
\widehat{\mathbf{D}}_{4}\end{array}$ & & $\begin{array}{r}\mathbf{C}_{2}^{\prime} \\
\mathbf{D}_{2 \widehat{I}}^{\prime}\end{array}$ & & & & $\begin{array}{c}\mathbf{C}_{3} \\
\mathbf{D}_{3 \widehat{I}}\end{array}$ & & $\begin{array}{l}\mathbf{C}_{2 \widehat{I}} \\
\mathbf{D}_{4 \widehat{I}}\end{array}$ & & & $\begin{array}{c}\widehat{\mathrm{D}}_{2}^{\prime} \\
\widehat{\mathrm{O}}\end{array}$ & $\begin{array}{l}\mathrm{C}_{4} \\
\mathrm{O}_{\widehat{I}}\end{array}$ & & $\mathrm{C}_{2 \hat{I}}^{\prime}$ & $\widehat{\mathbf{D}}_{2}^{\prime \prime}$ & $\mathbf{C}_{3 \widehat{I}}$ & ${ }_{\widehat{I}} \mathbf{D}_{3}$ & $\widehat{\mathbf{D}}_{3}$ & $\mathbf{D}_{4}$ \\
\hline \multirow[t]{2}{*}[8,0,0,0,0,0,0,0,0,0]{} & 0 & 0 & 0 & 0 & 0 & 0 & 0 & 0 & 0 & 0 & 0 & 0 & 0 & 0 & 0 & 0 & 0 & 0 & 0 & 0 \\
\hline & 0 & 0 & 0 & 0 & 0 & 0 & 0 & 0 & 0 & 0 & 0 & 0 & 1 & & & & & & & \\
\hline \multirow[t]{2}{*}[7,1,0,0,0,0,0,0,0,0]{} & 0 & 0 & 0 & 0 & 0 & 0 & 0 & 0 & 0 & 0 & 0 & 0 & 0 & 0 & 0 & 0 & 1 & 0 & 0 & 0 \\
\hline & 0 & 0 & 0 & 0 & 0 & 0 & 0 & 0 & 0 & 0 & 0 & 0 & 0 & & & & & & & \\
\hline \multirow{2}{*}[6,2,0,0,0,0,0,0,0,0]{} & 0 & 0 & 0 & 0 & 0 & 0 & 0 & 0 & 1 & 0 & 0 & 0 & 0 & 0 & 1 & 0 & 0 & 0 & 0 & 0 \\
\hline & 0 & 0 & 0 & 0 & 0 & 0 & 1 & 0 & 0 & 0 & 0 & 0 & 0 & & & & & & & \\
\hline \multirow[t]{2}{*}[6,1,1,0,0,0,0,0,0,0]{} & 0 & 0 & 0 & 2 & 0 & 0 & 0 & 0 & 0 & 0 & 0 & 0 & 0 & 0 & 0 & 0 & 1 & 0 & 0 & 0 \\
\hline & 0 & 0 & 0 & 0 & 0 & 0 & 0 & 0 & 0 & 0 & 0 & 0 & 0 & & & & & & & \\
\hline \multirow[t]{2}{*}[5,3,0,0,0,0,0,0,0,0]{} & 0 & 0 & 0 & 2 & 0 & 0 & 0 & 0 & 0 & 0 & 0 & 0 & 0 & 0 & 0 & 0 & 1 & 0 & 0 & 0 \\
\hline & 0 & 0 & 0 & 0 & 0 & 0 & 0 & 0 & 0 & 0 & 0 & 0 & 0 & & & & & & & \\
\hline \multirow[t]{2}{*}[5,2,1,0,0,0,0,0,0,0]{} & 0 & 0 & 0 & 7 & 0 & 0 & 0 & 0 & 0 & 0 & 0 & 0 & 0 & 0 & 0 & 0 & 0 & 0 & 0 & 0 \\
\hline & 0 & 0 & 0 & 0 & 0 & 0 & 0 & 0 & 0 & 0 & 0 & 0 & 0 & & & & & & & \\
\hline \multirow[t]{2}{*}[5,1,1,1,0,0,0,0,0,0]{} & 0 & 0 & 0 & 14 & 0 & 0 & 0 & 0 & 0 & 0 & 0 & 0 & 0 & 0 & 0 & 0 & 0 & 0 & 0 & 0 \\
\hline & 0 & 0 & 0 & 0 & 0 & 0 & 0 & 0 & 0 & 0 & 0 & 0 & 0 & & & & & & & \\
\hline \multirow[t]{2}{*}[4,4,0,0,0,0,0,0,0,0]{} & 0 & 0 & 0 & 1 & 0 & 0 & 0 & 0 & 0 & 0 & 0 & 0 & 0 & 0 & 2 & 0 & 1 & 0 & 0 & 0 \\
\hline & 0 & 1 & 1 & 0 & 0 & 0 & 0 & 0 & 0 & 0 & 1 & 0 & 0 & & & & & & & \\
\hline$[4,3,1,0,0,0,0,0,0,0]$ & 0 & 0 & 0 & 11 & 0 & 0 & 0 & 0 & 0 & 0 & 0 & 0 & 0 & 0 & 0 & 0 & 2 & 0 & 0 & 0 \\
\hline & 0 & 0 & 0 & 0 & 0 & 0 & 0 & 0 & 0 & 0 & 0 & 0 & 0 & & & & & & & \\
\hline$[4,2,2,0,0,0,0,0,0,0]$ & 0 & 0 & 0 & 13 & 0 & 0 & 0 & 0 & 3 & 0 & 0 & 0 & 0 & 0 & 6 & 0 & 0 & 0 & 0 & 0 \\
\hline & 0 & 0 & 0 & 0 & 0 & 0 & 0 & 0 & 0 & 0 & 0 & 0 & 0 & & & & & & & \\
\hline$[4,2,1,1,0,0,0,0,0,0]$ & 0 & 0 & 0 & 35 & 0 & 0 & 0 & 0 & 0 & 0 & 0 & 0 & 0 & 0 & 0 & 0 & 0 & 0 & 0 & 0 \\
\hline & 0 & 0 & 0 & 0 & 0 & 0 & 0 & 0 & 0 & 0 & 0 & 0 & 0 & & & & & & & \\
\hline$[4,1,1,1,1,0,0,0,0,0]$ & 0 & 0 & 0 & 70 & 0 & 0 & 0 & 0 & 0 & 0 & 0 & 0 & 0 & 0 & 0 & 0 & 0 & 0 & 0 & 0 \\
\hline & 0 & 0 & 0 & 0 & 0 & 0 & 0 & 0 & 0 & 0 & 0 & 0 & 0 & & & & & & & \\
\hline$[3,3,2,0,0,0,0,0,0,0]$ & 0 & 0 & 0 & 23 & 0 & 0 & 0 & 0 & 0 & 0 & 0 & 0 & 0 & 0 & 0 & 0 & 1 & 0 & 0 & 0 \\
\hline & 0 & 0 & 0 & 0 & 0 & 0 & 0 & 0 & 0 & 0 & 0 & 0 & 0 & & & & & & & \\
\hline$[3,3,1,1,0,0,0,0,0,0]$ & 0 & 0 & 0 & 46 & 0 & 0 & 0 & 0 & 0 & 0 & 0 & 0 & 0 & 0 & 0 & 0 & 2 & 0 & 0 & 0 \\
\hline & 0 & 0 & 0 & 0 & 0 & 0 & 0 & 0 & 0 & 0 & 0 & 0 & 0 & & & & & & & \\
\hline$[3,2,2,1,0,0,0,0,0,0]$ & 0 & 0 & 0 & 70 & 0 & 0 & 0 & 0 & 0 & 0 & 0 & 0 & 0 & 0 & 0 & 0 & 0 & 0 & 0 & 0 \\
\hline & 0 & 0 & 0 & 0 & 0 & 0 & 0 & 0 & 0 & 0 & 0 & 0 & 0 & & & & & & & \\
\hline$[3,2,1,1,1,0,0,0,0,0]$ & 0 & 0 & 0 & 140 & 0 & 0 & 0 & 0 & 0 & 0 & 0 & 0 & 0 & 0 & 0 & 0 & 0 & 0 & 0 & 0 \\
\hline & 0 & 0 & 0 & 0 & 0 & 0 & 0 & 0 & 0 & 0 & 0 & 0 & 0 & & & & & & & \\
\hline$[2,2,2,2,0,0,0,0,0,0]$ & 0 & 0 & 0 & 96 & 0 & 0 & 0 & 0 & 6 & 0 & 0 & 0 & 0 & 0 & 12 & 0 & 0 & 0 & 0 & 0 \\
\hline & 0 & 0 & 0 & 0 & 0 & 0 & 0 & 0 & 0 & 0 & 0 & 0 & 0 & & & & & & & \\
\hline$[2,2,2,1,1,0,0,0,0,0]$ & 0 & 0 & 0 & 210 & 0 & 0 & 0 & 0 & 0 & 0 & 0 & 0 & 0 & 0 & 0 & 0 & 0 & 0 & 0 & 0 \\
\hline & 0 & 0 & 0 & 0 & 0 & 0 & 0 & 0 & 0 & 0 & 0 & 0 & 0 & & & & & & & \\
\hline$[2,2,1,1,1,1,0,0,0,0]$ & 0 & 0 & 0 & 420 & 0 & 0 & 0 & 0 & 0 & 0 & 0 & 0 & 0 & 0 & 0 & 0 & 0 & 0 & 0 & 0 \\
\hline & 0 & 0 & 0 & 0 & 0 & 0 & 0 & 0 & 0 & 0 & 0 & 0 & 0 & & & & & & & \\
\hline$[7,0,0,0,0,0,1,0,0,0]^{*}$ & 0 & 0 & 0 & 0 & 0 & 0 & $1 / 2$ & 0 & 0 & 0 & 0 & 0 & 0 & 0 & 0 & 0 & 0 & 0 & 0 & 0 \\
\hline & 0 & 0 & 0 & 0 & 0 & 0 & 0 & 0 & 0 & 0 & 0 & 0 & 0 & & & & & & & \\
\hline$[6,1,0,0,0,0,1,0,0,0]^{*}$ & 1 & 0 & 0 & 0 & 0 & 0 & $1 / 2$ & 0 & 0 & 0 & & & & & & & & & & \\
\hline & 0 & 0 & 0 & 0 & 0 & 0 & 0 & 0 & 0 & 0 & & & & & & & & & & \\
\hline & 0 & 0 & 0 & 0 & 0 & 0 & 0 & 0 & 0 & 0 & 0 & 0 & 0 & & & & & & & \\
\hline$[6,0,0,0,0,0,2,0,0,0]^{*}$ & 0 & $1 / 2$ & $1 / 2$ & 0 & 0 & 0 & 0 & 0 & 0 & 0 & 0 & 0 & 0 & 0 & 0 & 0 & 0 & $1 / 2$ & 0 & 0 \\
\hline & 0 & 0 & 0 & 0 & 0 & 0 & 0 & 0 & 0 & 0 & 0 & 0 & 0 & & & & & & & \\
\hline$[6,0,0,0,0,0,1,1,0,0]$ & 0 & 0 & 0 & 0 & 1 & 1 & 0 & 0 & 0 & 0 & 0 & 0 & 0 & 0 & 0 & 0 & 0 & 0 & 1 & 0 \\
\hline & 0 & 0 & 0 & 0 & 0 & 0 & 0 & 0 & 0 & 0 & 0 & 0 & 0 & & & & & & & \\
\hline$[5,2,0,0,0,0,1,0,0,0]^{*}$ & $7 / 2$ & 0 & 0 & 0 & 0 & 0 & 0 & 0 & 0 & 0 & 0 & 0 & 0 & 0 & 0 & 0 & 0 & 0 & 0 & 0 \\
\hline & 0 & 0 & 0 & 0 & 0 & 0 & 0 & 0 & 0 & 0 & 0 & 0 & 0 & & & & & & & \\
\hline$[5,1,1,0,0,0,1,0,0,0]^{*}$ & 7 & 0 & 0 & 0 & 0 & 0 & 0 & 0 & 0 & 0 & 0 & 0 & 0 & 0 & 0 & 0 & 0 & 0 & 0 & 0 \\
\hline & 0 & 0 & 0 & 0 & 0 & 0 & 0 & 0 & 0 & 0 & 0 & 0 & 0 & & & & & & & \\
\hline $2,0,0,0]^{*}$ & $7 / 2$ & 0 & 0 & 0 & 0 & 0 & 0 & 0 & 0 & 0 & 0 & 0 & 0 & 0 & 0 & 0 & 0 & 0 & 0 & 0 \\
\hline & 0 & 0 & 0 & 0 & 0 & 0 & 0 & 0 & 0 & 0 & 0 & 0 & 0 & & & & & & & \\
\hline$[5,1,0,0,0,0,1,1,0,0]$ & 7 & 0 & 0 & 0 & 0 & 0 & 0 & 0 & 0 & 0 & 0 & 0 & 0 & 0 & 0 & 0 & 0 & 0 & 0 & 0 \\
\hline & 0 & 0 & 0 & 0 & 0 & 0 & 0 & 0 & 0 & 0 & 0 & 0 & 0 & & & & & & & \\
\hline$[5,0,0,0,0,0,3,0,0,0]^{*}$ & 1 & 0 & 0 & 0 & 0 & 0 & $1 / 2$ & 0 & 0 & 0 & 0 & 0 & 0 & 0 & 0 & 0 & 0 & 0 & 0 & 0 \\
\hline & 0 & 0 & 0 & 0 & 0 & 0 & 0 & 0 & 0 & 0 & 0 & 0 & 0 & & & & & & & \\
\hline$[5,0,0,0,0,0,2,1,0,0]^{*}$ & $7 / 2$ & 0 & 0 & 0 & 0 & 0 & 0 & 0 & 0 & 0 & 0 & 0 & 0 & 0 & 0 & 0 & 0 & 0 & 0 & 0 \\
\hline & 0 & 0 & 0 & 0 & 0 & 0 & 0 & 0 & 0 & 0 & 0 & 0 & 0 & & & & & & & \\
\hline$[5,0,0,0,0,0,1,1,1,0]^{*}$ & 7 & 0 & 0 & 0 & 0 & 0 & 0 & 0 & 0 & 0 & 0 & 0 & 0 & 0 & 0 & 0 & 0 & 0 & 0 & 0 \\
\hline & 0 & 0 & 0 & 0 & 0 & 0 & 0 & 0 & 0 & 0 & 0 & 0 & 0 & & & & & & & \\
\hline
\end{tabular}


Table 8. Cubane Derivatives as 3D Structural Isomers Under the LR-Permutation Group $\mathbf{O}_{\widehat{I}}$ (Part 2)

\begin{tabular}{|c|c|c|c|c|c|c|c|c|c|c|c|c|c|c|c|c|c|c|c|c|c|}
\hline & $\begin{array}{l}\mathbf{C}_{1} \\
\widehat{\mathbf{D}}_{4}\end{array}$ & $\begin{array}{c}\mathbf{C}_{2} \\
\mathbf{C}_{4 \widehat{I}}\end{array}$ & & $\begin{array}{l}\mathbf{C}_{\widehat{I}} \\
\mathbf{D}_{2 \widehat{I}}\end{array}$ & $\begin{array}{l}\widehat{\mathbf{C}}_{2} \\
\widehat{\mathbf{D}}^{\prime \prime}\end{array}$ & & $\begin{array}{c}\mathrm{C}_{3} \\
\mathrm{D}_{3 \widehat{I}}\end{array}$ & $\begin{array}{c}\mathbf{D}_{2} \\
\mathbf{T}\end{array}$ & $\begin{array}{l}\mathbf{C}_{2 \widehat{I}} \\
\mathbf{D}_{4 \hat{I}}\end{array}$ & & $\mathbf{T}_{\widehat{I}}$ & $\begin{array}{c}\mathrm{D}_{2}^{\prime} \\
\widehat{\mathrm{O}}\end{array}$ & $\begin{array}{l}\mathrm{C}_{4} \\
\mathrm{O}_{\widehat{I}}\end{array}$ & D & & & & & & & $\mathbf{D}_{4}$ \\
\hline \multirow[t]{2}{*}[4,0,0,0,0,0,4,0,0,0]{$^{*}$} & $1 / 2$ & 0 & 1 & 0 & 0 & 0 & $1 / 2$ & 0 & 0 & & 0 & 0 & $1 / 2$ & $1 / 2$ & 0 & & & & 0 & 0 & 0 \\
\hline & 0 & 0 & 0 & 0 & 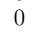 & 0 & 0 & $1 / 2$ & & & O & 0 & 0 & & & & & & & & \\
\hline \multirow[t]{2}{*}[4,3,0,0,0,0,1,0,0,0]{$^{*}$} & $11 / 2$ & 0 & 0 & 0 & U & 0 & 1 & 0 & & & 0 & 0 & 0 & 0 & 0 & & & & 0 & 0 & 0 \\
\hline & 0 & 0 & 0 & 0 & 0 & 0 & 0 & 0 & & 0 & 0 & 0 & 0 & & & & & & & & \\
\hline \multirow[t]{2}{*}[4,2,0,0,0,0,2,0,0,0]{$^{*}$} & $13 / 2$ & $3 / 2$ & 3 & 0 & 0 & 0 & 0 & 0 & 0 & 0 & 0 & 0 & 0 & 0 & 0 & 0 & 0 & & 0 & 0 & 0 \\
\hline & 0 & 0 & 0 & 0 & 0 & 0 & 0 & 0 & 0 & 0 & 0 & 0 & 0 & & & & & & & & \\
\hline \multirow{2}{*}[4,0,0,0,0,0,2,2,0,0]{} & 4 & 0 & 2 & 0 & 1 & 4 & 0 & 0 & 0 & 1 & 1 & 1 & 0 & 0 & 0 & 2 & 0 & & 0 & 0 & 0 \\
\hline & 0 & 0 & 0 & 0 & 0 & 0 & 0 & 0 & 0 & 0 & 0 & 0 & 0 & & & & & & & & \\
\hline \multirow{2}{*}[4,0,0,0,0,0,2,0,2,0]{$^{*}$} & $13 / 2$ & $3 / 2$ & 3 & . & 0 & 0 & 0 & 0 & 0 & 0 & 0 & 0 & 0 & 0 & 0 & & 8 & & 0 & 0 & 0 \\
\hline & 0 & 0 & 0 & 0 & 0 & 0 & 0 & 0 & $\theta$ & $\Omega$ & 0 & 0 & 0 & & & & & & & & \\
\hline \multirow[t]{2}{*}[4,2,1,0,0,0,1,0,0,0]{$^{*}$} & $35 / 2$ & 0 & 0 & . & ( & 0 & 0 & 0 & 0 & 0 & 0 & 0 & 0 & 0 & 0 & & 8 & & 0 & 0 & 0 \\
\hline & 0 & 0 & 0 & 0 & 0 & 0 & 0 & 0 & 0 & 0 & 0 & 0 & 0 & & & & & & & & \\
\hline \multirow[t]{2}{*}[4,2,0,0,0,0,1,1,0,0]{} & 13 & 0 & 0 & 0 & 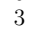 & 6 & 0 & 0 & 0 & 0 & 0 & 0 & 0 & 0 & 0 & & O & & 0 & 0 & 0 \\
\hline & 0 & 0 & 0 & 0 & 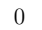 & 0 & 0 & 0 & 0 & 0 & 0 & 0 & 0 & & & & & & & & \\
\hline \multirow[t]{2}{*}[4,1,1,1,0,0,1,0,0,0]{$^{*}$} & 35 & 0 & 0 & 0 & 0 & 0 & 0 & 0 & 0 & 0 & 0 & 0 & 0 & 0 & 0 & & & & 0 & 0 & 0 \\
\hline & 0 & 0 & 0 & 0 & . & 0 & 0 & 0 & 0 & 0 & 0 & 0 & 0 & & & & & & & & \\
\hline \multirow[t]{2}{*}[4,1,1,0,0,0,1,1,0,0]{} & 35 & 0 & 0 & 0 & 0 & 0 & 0 & 0 & 0 & 0 & 0 & 0 & 0 & 0 & 0 & & & & 0 & 0 & 0 \\
\hline & 0 & 0 & 0 & 0 & 0 & 0 & 0 & 0 & & ( & 0 & 0 & 0 & & & & & & & & \\
\hline$[4,1,0,0,0,0,1,1,1,0]^{*}$ & 35 & 0 & 0 & 0 & 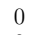 & 0 & 0 & 0 & 0 & ( & 0 & 0 & 0 & 0 & 0 & & & & 0 & 0 & 0 \\
\hline & 0 & 0 & 0 & c & o & . & 0 & & & & 0 & 0 & 0 & & & & & & & & \\
\hline$[4,0$, & 26 & 0 & 0 & 0 & 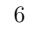 & 12 & 0 & 0 & & c & 0 & 0 & 0 & 0 & 0 & & & & 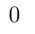 & 0 & 0 \\
\hline & 0 & 0 & 0 & 0 & 0 & 0 & O & & & & 0 & & 0 & & & & & & & & \\
\hline$[3,3$, & 23 & 0 & 0 & 0 & ( & 0 & 1 & 0 & 0 & 0 & 0 & 0 & 0 & 0 & U & & 0 & & 0 & 0 & 0 \\
\hline & 0 & 0 & 0 & 0 & . & 0 & 0 & & & 0 & 0 & 0 & 0 & & & & & & & & \\
\hline$[3,3$, & 23 & 0 & 0 & 0 & 0 & 0 & 1 & 0 & 0 & 0 & 0 & 0 & 0 & 0 & U & & & & 0 & 0 & 0 \\
\hline & 0 & 0 & 0 & 0 & 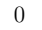 & 0 & 0 & 0 & 0 & 0 & 0 & 0 & 0 & & & & & & & & \\
\hline$[3,3$ & $23 / 2$ & 0 & 0 & 0 & 0 & 0 & $1 / 2$ & 0 & 0 & 0 & 0 & 0 & 0 & 0 & 0 & & & & 0 & 0 & 0 \\
\hline & 0 & 0 & 0 & 0 & 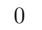 & 0 & 0 & 0 & & & 0 & 0 & 0 & & & & & & & & \\
\hline$[3,2$ & 35 & 0 & 0 & 0 & ( & 0 & 0 & 0 & 0 & ( & 0 & 0 & 0 & 0 & . & & & & 0 & 0 & 0 \\
\hline & 0 & 0 & 0 & 0 & 0 & 0 & 0 & 0 & & & 0 & & O & & & & & & & & \\
\hline$[3,2$, & 35 & 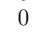 & 0 & 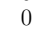 & 0 & 0 & . & 0 & 0 & 0 & 0 & 0 & 0 & 0 & 0 & & & & 0 & 0 & 0 \\
\hline & 0 & 0 & 0 & 0 & 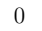 & 0 & 0 & 0 & & & 0 & & 0 & & & & & & & & \\
\hline$[3,2$, & 70 & 0 & 0 & 0 & 0 & 0 & 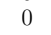 & 0 & 0 & ( & 0 & 0 & 0 & 0 & 0 & & & & 0 & 0 & 0 \\
\hline & 0 & 0 & 0 & 0 & 0 & 0 & 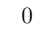 & & & & 0 & & & & & & & & & & \\
\hline$[3,2$, & 70 & 0 & 0 & 0 & 0 & 0 & 0 & 0 & c & 0 & 0 & 0 & 0 & 0 & 0 & & & & 0 & 0 & 0 \\
\hline & 0 & 0 & 0 & 0 & ( & 0 & 0 & & & & 0 & & & & & & & & & & \\
\hline$[3,1$ & 0 & 0 & 0 & 280 & 0 & 0 & 0 & 0 & 0 & 0 & 0 & 0 & 0 & 0 & 0 & & & & 0 & 0 & 0 \\
\hline & 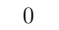 & c & 0 & & ( & 0 & 0 & & & & & & & & & & & & & & \\
\hline$[3,1$, & 140 & 0 & 0 & ( & 0 & 0 & 0 & 0 & 0 & 0 & 0 & 0 & 0 & 0 & 0 & & & & 0 & 0 & 0 \\
\hline & 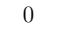 & c & 0 & & ( & ( & c & & & & 0 & & & & & & & & & & \\
\hline$[3,1$, & 140 & 0 & 0 & ( & 0 & 0 & 0 & 0 & & 0 & 0 & 0 & 0 & 0 & 0 & & & & 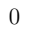 & 0 & 0 \\
\hline & 0 & 0 & 0 & & 0 & 0 & 0 & 0 & 0 & 0 & 0 & 0 & 0 & & & & & & & & \\
\hline$[3,1$ & 140 & 0 & 0 & ( & 0 & 0 & 0 & 0 & 0 & 0 & & & & & & & & & & & \\
\hline & 0 & 0 & 0 & & 0 & 0 & 0 & & & 0 & & & & & & & & & & & \\
\hline & . & c & 0 & & 0 & 0 & 0 & c & & 0 & 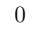 & 0 & & & & & & & & & \\
\hline$[3,1$, & 140 & 0 & 0 & 0 & 0 & 0 & 0 & 0 & c & 0 & 0 & 0 & 0 & 0 & to & & & & 0 & 0 & 0 \\
\hline & 0 & 0 & 0 & 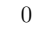 & 0 & 0 & 0 & c & & 0 & 0 & 0 & 0 & & & & & & & & \\
\hline$[3$ & $23 / 2$ & 0 & 0 & 0 & 0 & 0 & $1 / 2$ & 0 & 0 & 0 & 0 & 0 & 0 & 0 & 0 & & & & 0 & 0 & 0 \\
\hline & 0 & 0 & 0 & 0 & 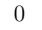 & 0 & o & 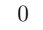 & & & 0 & 0 & 0 & & & & & & & & \\
\hline$[3,0$, & 23 & 0 & 0 & 0 & 0 & 0 & 1 & 0 & 0 & 0 & 0 & 0 & 0 & 0 & 0 & & & & 0 & 0 & 0 \\
\hline & 0 & 0 & 0 & 0 & 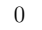 & 0 & 0 & 0 & & ( & 0 & 0 & 0 & & & & & & & & \\
\hline$[3,0$, & 35 & 0 & 0 & 0 & 0 & 0 & 0 & 0 & 0 & 0 & 0 & 0 & 0 & 0 & 0 & & 5 & & 0 & 0 & 0 \\
\hline & 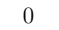 & 0 & 0 & 0 & 0 & 0 & 0 & 0 & & ( & 0 & 0 & 0 & & & & & & & & \\
\hline$[3,0,0$ & 70 & 0 & 0 & 0 & 0 & 0 & 0 & 0 & 0 & 0 & 0 & 0 & 0 & 0 & 0 & & 0 & & 0 & 0 & 0 \\
\hline & 0 & 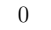 & . & 0 & 0 & 0 & 0 & 0 & & ( & 0 & J & 0 & & & & & & & & \\
\hline$[3,2,1,1,0,0,1,0,0,0]^{*}$ & 70 & 0 & 0 & 0 & 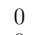 & 0 & 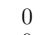 & 0 & 0 & 0 & 0 & 0 & 0 & 0 & 0 & 0 & 0 & & 0 & 0 & 0 \\
\hline & 0 & 0 & 0 & 0 & 0 & 0 & U & 0 & 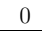 & 0 & 0 & 8 & & & & & & & & & \\
\hline
\end{tabular}


Table 9. Cubane Derivatives as 3D Structural Isomers Under the $L R$-Permutation Group $\mathbf{O}_{\widehat{I}}$ (Part 3)

\begin{tabular}{|c|c|c|c|c|c|c|c|c|c|c|c|c|c|c|c|c|c|c|c|c|}
\hline part & $\widehat{\mathbf{D}}_{4}$ & & & $\mathbf{D}_{2 \widehat{I}}$ & & & )$_{\widehat{A}}$ & $\mathbf{T}$ & $\begin{array}{l}\mathbf{C}_{2 \widehat{I}} \\
\mathbf{D}_{4 \widehat{I}}\end{array}$ & $\mathrm{O}$ & $\mathbf{T}_{\widehat{I}}$ & $\begin{array}{l}\widehat{\mathbf{D}}_{2}^{\prime} \\
\widehat{\mathbf{O}}\end{array}$ & $\begin{array}{l}\mathrm{C}_{4} \\
\mathrm{O}_{\widehat{I}}\end{array}$ & & & & & & & 1 \\
\hline \multirow[t]{2}{*}[2,2,2,0,0,0,2,0,0,0]{$^{*}$} & 4 & 3 & 6 & 0 & 0 & 0 & 0 & 0 & 0 & 0 & 0 & 0 & 0 & 0 & 0 & 0 & 0 & 0 & 0 & 0 \\
\hline & 0 & 0 & & 0 & 0 & 0 & 0 & 0 & & & 0 & & 0 & & & & & & & \\
\hline \multirow[t]{2}{*}[2,2,0,0,0,0,2,2,0,0]{} & 39 & 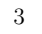 & & 0 & 6 & 12 & 0 & 0 & . & 0 & 0 & 0 & 0 & 0 & 0 & 0 & 0 & 0 & 0 & \\
\hline & 0 & 0 & 0 & 0 & 0 & 0 & 0 & 0 & 0 & 0 & 0 & 0 & 0 & & & & & & & \\
\hline \multirow[t]{2}{*}[2,0,0,0,0,0,2,2,2,0]{$^{*}$} & 48 & 3 & 0 & 0 & 0 & 0 & 0 & 0 & 0 & 0 & 0 & 0 & 0 & 0 & 0 & 0 & 0 & 0 & 0 & U \\
\hline & 0 & 0 & 0 & 0 & 0 & 0 & . & 0 & 0 & 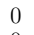 & 0 & 0 & 0 & & & & & & & \\
\hline \multirow[t]{2}{*}[2,2,2,1,0,0,1,0,0,0]{$^{*}$} & 105 & 0 & 0 & 0 & 0 & 0 & 0 & 0 & 0 & 0 & 0 & U & 0 & 0 & 0 & 0 & 0 & 0 & 0 & 0 \\
\hline & 0 & 0 & 0 & 0 & 0 & 0 & & 0 & 0 & 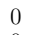 & 0 & 0 & 0 & & & & & & & \\
\hline \multirow[t]{2}{*}[2,2,2,0,0,0,1,1,0,0]{} & 96 & 0 & & 0 & 6 & 12 & & 0 & & 0 & 0 & 0 & 0 & 0 & 0 & 0 & 0 & 0 & 0 & \\
\hline & 0 & 0 & & 0 & 0 & 0 & & & & & 0 & & 0 & & & & & & & \\
\hline \multirow[t]{2}{*}[2,2,1,1,1,0,1,0,0,0]{$^{*}$} & 210 & 0 & 1 & 0 & 0 & 0 & 0 & 0 & & 0 & 0 & 0 & 0 & 0 & 0 & 0 & 0 & 0 & 0 & 0 \\
\hline & 0 & r & & 0 & 0 & 0 & & & & & 0 & & 0 & & & & & & & \\
\hline \multirow[t]{2}{*}[2,2,1,1,0,0,1,1,0,0]{} & 210 & 0 & & 0 & 0 & 0 & 0 & 0 & 0 & 0 & 0 & 0 & 0 & 0 & 0 & 0 & 0 & 0 & 0 & 0 \\
\hline & 0 & 0 & ( & 0 & 0 & 0 & & & & & 0 & 0 & 0 & & & & & & & \\
\hline \multirow[t]{2}{*}[2,2,1,0,0,0,1,1,1,0]{$^{*}$} & 210 & 0 & 0 & 0 & 0 & 0 & 0 & 0 & 0 & 0 & 0 & 0 & 0 & 0 & 0 & 0 & 0 & 0 & U & U \\
\hline & 0 & 0 & & 0 & 0 & 0 & & & & & 0 & 0 & 0 & & & & & & & \\
\hline \multirow[t]{2}{*}[2,2,0,0,0,0,1,1,1,1]{} & 192 & 0 & 0 & 0 & 12 & 24 & 0 & 0 & 0 & 0 & 0 & 0 & 0 & 0 & 0 & 0 & 0 & 0 & 0 & 0 \\
\hline & 0 & 0 & 0 & 0 & 0 & 0 & 0 & & & & 0 & 0 & 0 & & & & & & & \\
\hline \multirow[t]{2}{*}[2,1,1,1,1,1,1,0,0,0]{$^{*}$} & 420 & 0 & 0 & 0 & 0 & 0 & 0 & 0 & 0 & 0 & 0 & 0 & 0 & 0 & 0 & 0 & 0 & 0 & 0 & 0 \\
\hline & 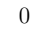 & 0 & 0 & 0 & 0 & 0 & ( & & & & 0 & 0 & 0 & & & & & & & \\
\hline \multirow[t]{2}{*}[2,1,1,1,1,0,1,1,0,0]{} & 420 & 0 & 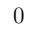 & 0 & 0 & 0 & 0 & 0 & 0 & 0 & 0 & 0 & 0 & 0 & 0 & 0 & 0 & 0 & 0 & 0 \\
\hline & 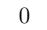 & 0 & 0 & O & 0 & 0 & & & & & 0 & & 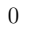 & & & & & & & \\
\hline \multirow[t]{2}{*}[2,1,1,1,0,0,1,1,1,0]{$^{*}$} & 420 & 0 & 0 & 0 & 0 & 0 & 0 & 0 & 0 & 0 & 0 & 0 & 0 & 0 & 0 & 0 & 0 & 0 & 0 & 0 \\
\hline & 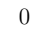 & 0 & 0 & 0 & 0 & 0 & & & & & 0 & & 0 & & & & & & & \\
\hline \multirow[t]{2}{*}[2,1,1,0,0,0,1,1,1,1]{} & 420 & 0 & 0 & 0 & 0 & 0 & c & 0 & 0 & 0 & 0 & 0 & 0 & 0 & 0 & 0 & 0 & 0 & c & 0 \\
\hline & 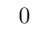 & 0 & 0 & 0 & 0 & 0 & & & & & 0 & & 0 & & & & & & & \\
\hline \multirow{2}{*}[1,1,1,1,1,1,2,0,0,0]{$^{*}$} & 420 & 0 & 0 & 0 & 0 & 0 & 0 & 0 & 0 & 0 & 0 & 0 & 0 & 0 & 0 & 0 & 0 & 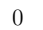 & 0 & 0 \\
\hline & 0 & 0 & 0 & 0 & 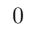 & 0 & & & & & . & 0 & 0 & & & & & & & \\
\hline \multirow[t]{2}{*}[1,1,1,1,1,0,2,1,0,0]{$^{*}$} & 420 & 0 & 0 & 0 & 0 & 0 & 0 & 0 & 0 & 0 & 0 & 0 & 0 & 0 & 0 & 0 & 0 & 0 & 0 & 0 \\
\hline & - & 0 & 0 & & 0 & & & & & & 0 & & 0 & & & & & & & \\
\hline \multirow[t]{2}{*}[1,1,1,1,0,0,2,1,1,0]{} & 420 & 0 & 0 & 0 & 0 & 0 & 0 & 0 & 0 & 0 & 0 & 0 & 0 & 0 & 0 & 0 & 0 & 0 & 0 & 0 \\
\hline & 0 & 0 & c & 0 & 0 & 0 & 0 & & & 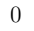 & 0 & 0 & 0 & & & & & & & \\
\hline \multirow[t]{2}{*}[1,1,1,0,0,0,2,1,1,1]{$^{*}$} & 420 & 0 & 0 & 0 & 0 & 0 & 0 & 0 & 0 & 0 & 0 & 0 & 0 & 0 & 0 & 0 & 0 & 0 & 0 & 0 \\
\hline & 0 & 0 & 0 & 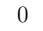 & 0 & 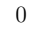 & 0 & & & 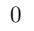 & 0 & 0 & 0 & & & & & & & \\
\hline \multirow[t]{2}{*}[1,1,1,1,1,1,1,1,0,0]{} & 840 & 0 & 0 & 0 & 0 & 0 & 0 & 0 & 0 & 0 & 0 & 0 & 0 & 0 & 0 & 0 & 0 & 0 & 0 & 0 \\
\hline & 0 & 0 & 0 & 0 & 0 & 0 & 0 & & 0 & 0 & 0 & 0 & 0 & & & & & & & \\
\hline$[1,1,1,1,1,0,1,1,1,0]^{*}$ & 840 & 0 & 0 & 0 & 0 & 0 & 0 & 0 & 0 & 0 & 0 & 0 & 0 & 0 & 0 & U & 0 & 0 & 0 & 0 \\
\hline & 0 & 0 & 0 & 0 & 0 & 0 & 0 & 0 & 0 & 0 & 0 & 0 & 0 & & & & & & & \\
\hline$[1,1$, & 840 & 0 & 0 & 0 & 0 & 0 & 0 & 0 & 0 & 0 & 0 & 0 & 0 & 0 & 0 & 0 & 0 & 0 & 0 & 0 \\
\hline & 0 & 0 & 0 & 0 & 0 & 0 & 0 & 0 & 0 & U & 0 & 0 & 0 & & & & & & & \\
\hline
\end{tabular}




\begin{tabular}{|c|c|c|}
\hline & $R S$-astereogenic ( $R S$-non-stereogenic) & $R S$-stereogenic \\
\hline \multirow{2}{*}{ chiral } & & 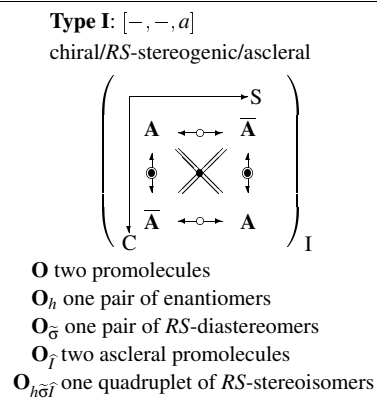 \\
\hline & 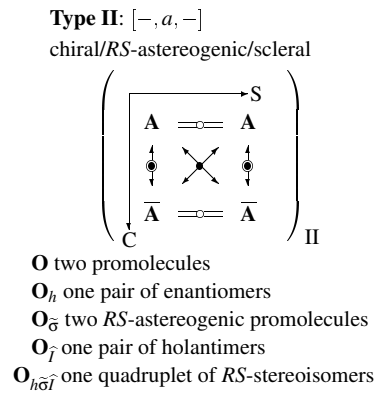 & 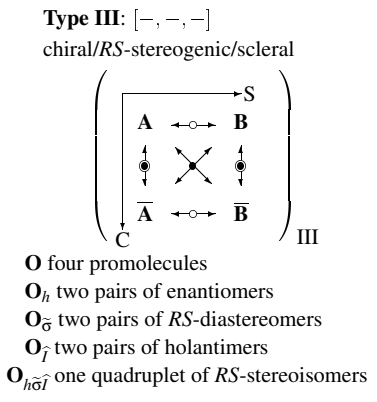 \\
\hline achiral & 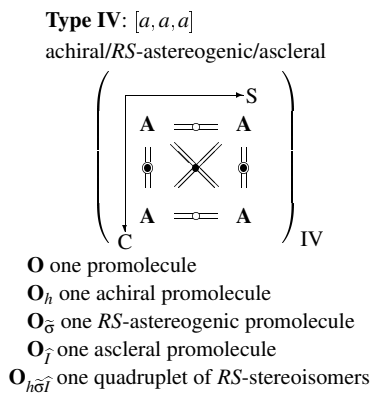 & 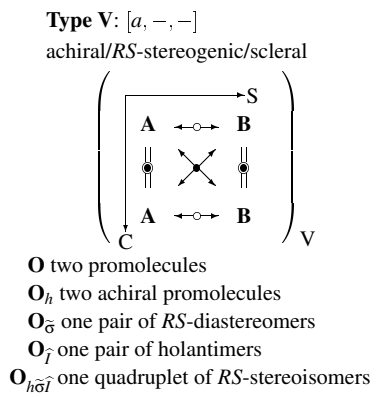 \\
\hline
\end{tabular}

Figure 5. Stereoisograms for representing $R S$-stereoisomers of five types, each of which is a modification of the diagram reported in a previous article [9]. Each type is designated by a Roman-numeral subscript attached below a closing parenthesis. The rows below each stereoisogram indicate the respective entities, which are counted once or twice under the point group $\mathbf{O}_{h}$, the $R S$-stereogenic group $\mathbf{O}_{\tilde{\sigma}}$, the $L R$-permutation group $\mathbf{O}_{\widehat{I}}$, and the $R S$-stereoisomeric group $\mathbf{O}_{h \widetilde{\sigma} \widehat{I}}$. 
been generalized into Figure 4 of a previous article reported in this journal [40], which has given a general proof for the existence of five stereoisogram types on the basis of the existence of five types of subgroups of $R S$-stereoisomeric groups. The generalized chart [40] has been further improved by adding the number of unit promolecules (orbits) assigned to each of the three aspects of a stereoisogram [9, 26]. This improved chart (Figure 12 of the more recent reference [26]) has been slightly modified to give Figure 5 by adding the roles of $\mathbf{O}, \mathbf{O}_{h}, \mathbf{O}_{\widetilde{\sigma}}, \mathbf{O}_{\widehat{I}}$, and $\mathbf{O}_{h \widetilde{\sigma} \widehat{I}}$.

In Figure 5, the symbols $\mathbf{A}$ and $\overline{\mathbf{A}}$ (or $\mathbf{B}$ and $\overline{\mathbf{B}}$ ) represent a pair of enantiomeric promolecules. Each stereoisogram consists of a quadruplet of $R S$-stereoisomeric promolecules, which may coalesce with one another according to either one of the five $R S$ stereoisomeric types. The five $R S$-stereoisomeric types are represented by type indices, in which a letter a means "achiral", "RS-astereogenic", or "ascleral", while a letter means "chiral", "RS-stereogenic", or "scleral". For example, a type-V stereoisogram is represented by the type index $[a,-,-]$, which means [achiral, $R S$-stereogenic, scleral], where the achirality is characterized by the presence of vertical equality symbols in the type-V stereoisogram. A type-II stereoisogram $[-, a,-]$ is characterized by the presence of horizontal equality symbols and a type-I stereoisogram $[-,-, a]$ is characterized by the presence of diagonal equality symbols. As one extreme case, the four $R S$-stereoisomeric promolecules of a Type-III stereoisogram are different (i.e., $\mathbf{A}, \overline{\mathbf{A}}, \mathbf{B}$ and $\overline{\mathbf{B}}$ ), as shown by the type index $[-,-,-]$. The other extreme case is a Type-IV stereoisogram, which consists of a degenerate $R S$-stereoisomer (i.e., A), as shown by the type index $[a, a, a]$.

By means of the symmetry-itemized enumerations introduced in the present article, type indices can be further sophisticated to reach $R S$-stereoisomeric indices. For example, a type index $[a,-,-]$ for a type-V stereoisogram can be replaced by a more itemized description such as an $R S$-stereoisomeric index $\left[\left[\mathbf{C}_{s}^{\prime}, \mathbf{C}_{1}, \mathbf{C}_{1}\right]\right]$. Thus, "achirality" in $[a,-,-]$ is replaced by the point-group symbol " $\mathbf{C}_{s}^{\prime \prime}$ " in $\left[\left[\mathbf{C}_{s}^{\prime}, \mathbf{C}_{1}, \mathbf{C}_{1}\right]\right]$.

It should be noted that Figure 5 is divided into four parts by means of a double vertical line and a double horizontal line. Thereby, "chirality" is concerned with Type I, II, and III (younger-numbered types in the upper part of Figure 5), while achirality is concerned with Type IV and V (older-numbered types in the lower part of Figure 5). On the other hand, "RS-stereogenicity" is concerned with Type I, III, and V (odd-numbered types in the right part of Figure 5), while $R S$-astereogenicity ( $R S$-non-stereogenicity) is concerned 
with II and IV (even-numbered types in the left part of Figure 5).

\section{Discussions on examples of symmetry-itemized enumerations of cubane derivatives}

This section adopts several examples of symmetry-itemized enumerations of cubane derivatives, where the three aspects of symmetry i.e., chirality/achirality, $R S$-stereogenicity/ $R S$ astereogenicity, and sclerality/asclerality, are discussed by examining symmetry-itemized enumerations under the point group $\mathbf{O}_{h}$ (Tables 1-3), under the $R S$-stereogenic group $\mathbf{O}_{\widetilde{\sigma}}$ (Tables 4-6), and under the $L R$-permutation group $\mathbf{O}_{\widehat{I}}$ (Tables 7-9), respectively, where the $R S$-stereoisomeric group $\mathbf{O}_{h \widetilde{\sigma} \widehat{I}}$ is used as an integrated group for controlling five-types of stereoisograms of a cubane skeleton.

\subsection{Disubstituted cubane derivatives}

\subsubsection{Cubane derivatives with composition $\mathrm{H}_{6} \mathrm{~A}_{2}$}

The $[6,2,0,0,0,0,0,0,0,0]$-row of Table 1 indicates the presence of the value 1 at the $\mathbf{C}_{2 v^{-}}^{\prime}$-column (the 13 th column), the $\mathbf{C}_{2 v}^{\prime \prime}$-column (the 14 th column), and the $\mathbf{D}_{3 d^{-}}$-column (the 28th column) under the point group $\mathbf{O}_{h}$. They are depicted as $\mathbf{4}\left(\mathbf{C}_{2 v}^{\prime}\right), \mathbf{6}\left(\mathbf{C}_{2 v}^{\prime \prime}\right)$, and $8\left(\mathbf{D}_{3 d}\right)$ in the type-IV stereoisograms of Figure 6. Because one pair of enantiomers or one pair of self-enantiomers (i.e., one achiral promolecule) is counted once under the point group $\mathbf{O}_{h}$, they are depicted along the vertical direction. Because there appear achiral derivatives, they are depicted by simplified expressions, i.e., $([\mathbf{4}])_{\mathrm{IV}},([\mathbf{6}])_{\mathrm{IV}}$, and $([\mathbf{8}])_{\mathrm{IV}}$. In this enumeration, one pair of self-enantiomers (an achiral promolecule) is linked by a vertical equality symbol with an encircled bullet $(=0)$. It should be emphasized that the point group $\mathbf{O}_{h}$ is concerned with a pair of self-enantiomers $([4(=\overline{4})])_{\mathrm{IV}},([\mathbf{6}(=\overline{\mathbf{6}})])_{\mathrm{IV}}$, or $([\mathbf{8}(=\overline{\mathbf{8}})])_{\mathrm{IV}}$, although the achirality aspect results in the presence of a vertical equality symbol.

The $[6,2,0,0,0,0,0,0,0,0]$-row of Table 3 of the previous gross-enumeration [26] reported the total number 3 of cubane derivatives of the composition $\mathrm{H}_{6} \mathrm{~A}_{2}$ under the point group $\mathbf{O}_{h}$ and the presence of 3 stereoisograms under the $R S$-stereoisomeric group $\mathbf{O}_{h \widetilde{\sigma} \widehat{I}}$. These gross values are consistent with the present symmetry-itemized enumeration.

The $[6,2,0,0,0,0,0,0,0,0]$-row of Table 4 indicates the presence of the value 1 at the $\widetilde{\mathbf{C}}_{2 v}^{\prime}$-column (the 13 th column), the $\widetilde{\mathbf{C}}_{2 v}^{\prime \prime}$-column (the 14 th column), and the $\widetilde{\mathbf{D}}_{3 d^{-}}$-column 


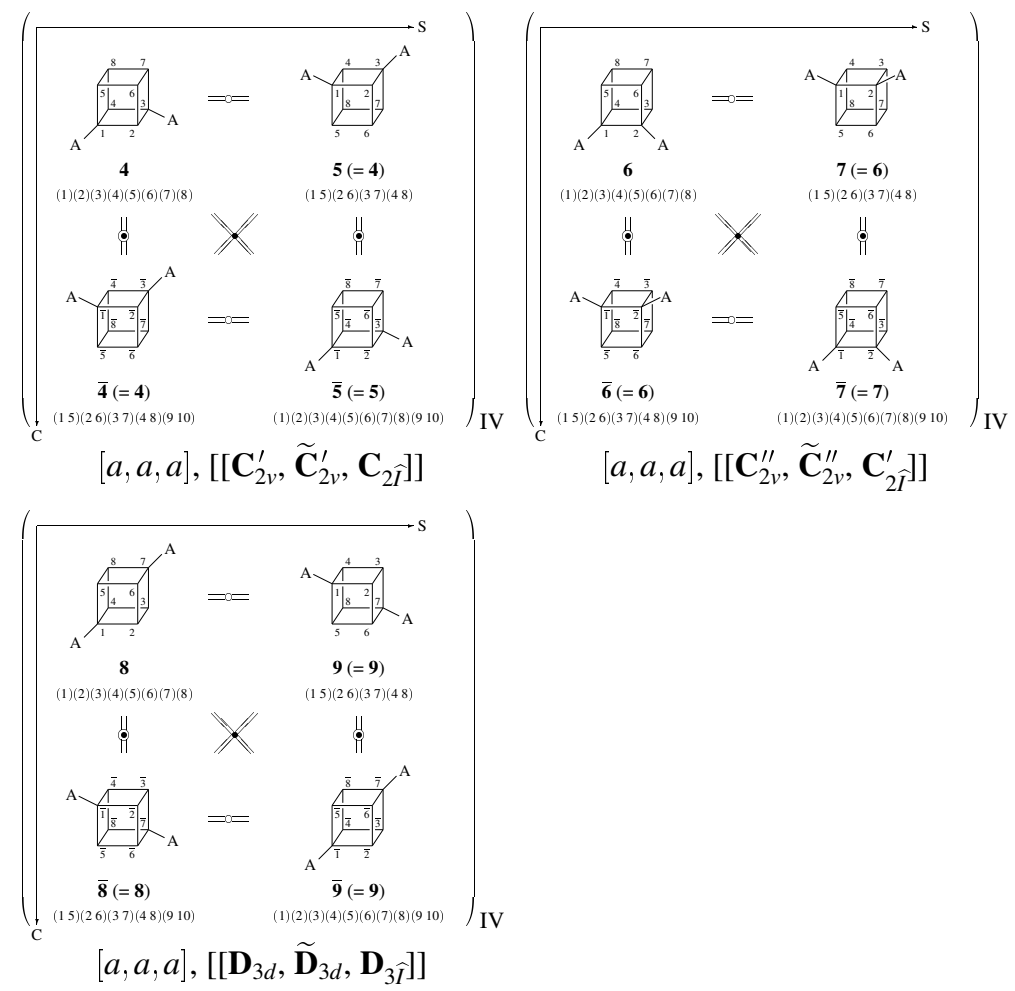

Figure 6. Stereoisograms of cubane derivative with the composition $\mathrm{H}_{6} \mathrm{~A}_{2}$. The type index $[a, a, a]$ is assigned to such a type-IV stereoisogram, which is specified to be achiral, $R S$-astereogenic, and ascleral.

(the 28th column) under the $R S$-stereogenic group $\mathbf{O}_{\widetilde{\sigma}}$. They are depicted as $\mathbf{5}\left(\widetilde{\mathbf{C}}_{2 v}^{\prime}\right)$, $7\left(\widetilde{\mathbf{C}}_{2 v}^{\prime \prime}\right)$, and $\mathbf{9}\left(\widetilde{\mathbf{D}}_{3 d}\right)$ in the type-IV sterereoisograms of Figure 6 . Because one pair of $R S$-diastereomers or one piar of self- $R S$-diastereomers (i.e., one $R S$-astereogenic or $R S$ non-stereogenic promolecule) is counted once under the $R S$-stereogenic group $\mathbf{O}_{\tilde{\sigma}}$, they are depicted along the horizontal direction, e.g., $([[\mathbf{4}(=\mathbf{5})]]) \mathrm{IV}$. In this enumeration, the pair of $\mathbf{4}$ and $\mathbf{5}$ is linked by a horizontal equality symbol with a circle $(=\mathbf{E})$ and regarded to be identical with each other under the $R S$-stereogenic group $\mathbf{O}_{\tilde{\sigma}}$. Hence, $([[\mathbf{4}]])_{\mathrm{IV}}$ is selected, because the presence of a horizotal equality symbol. As a result, one pair of self$R S$-diastereomers (an $R S$-astereogenic promolecule) i.e., $([[\mathbf{4}]])_{\mathrm{IV}}$ as a $\widetilde{\mathbf{C}}_{2 v}^{\prime}$-promolecule, $([[\mathbf{6}]])_{\mathrm{IV}}$ as a $\widetilde{\mathbf{C}}_{2 v}^{\prime \prime}$-promolecule, or $([[\mathbf{8}]])_{\mathrm{IV}}$ as a $\widetilde{\mathbf{D}}_{3 d}$-promolecule, is counter once under 
the $R S$-stereogenic group $\mathbf{O}_{\widetilde{\sigma}}$.

The $[6,2,0,0,0,0,0,0,0,0]$-row of Table 7 indicates the presence of the value 1 at the

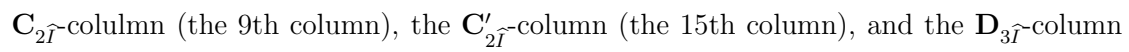
(the 27th column) under the $L R$-permutation group $\mathbf{O}_{\widehat{I}}$. They are depicted as $\overline{\mathbf{5}}\left(\mathbf{C}_{2 \widehat{I}}\right)$, $\overline{\mathbf{7}}\left(\mathbf{C}_{2 \widehat{I}}^{\prime}\right)$, and $\overline{\mathbf{9}}\left(\mathbf{D}_{3 \widehat{I}}\right)$ in the type-IV sterereoisograms of Figure 6 . Because one pair of (self-)holantimers is counted once under the $L R$-permutation group $\mathbf{O}_{\widehat{I}}$, they are depicted along the diagonal direction, $(<[\mathbf{4}(=\overline{\mathbf{5}})]>)_{\mathrm{IV}},(<[\mathbf{6}(=\overline{\mathbf{7}})]>)_{\mathrm{IV}}$, and $(<[\mathbf{8}(=\overline{\mathbf{9}})]>)_{\mathrm{IV}}$. In this enumeration, one pair of self-holantimers (an ascleral promolecule) is linked by a diagonal equality symbol with a bullet $(=\mathbf{0}=)$ so that we simply specify $(<[\mathbf{4}]>)_{\mathrm{IV}}$, $(<[6]>)_{\mathrm{IV}}$, and $(<[8]>)_{\mathrm{IV}}$.

Each of the type-IV stereoisograms depicted in Figure 6 is characterized by the type index $[a, a, a]$, which means achiral, $R S$-astereogenic, and ascleral. By the present symmetry-itemized enumeration, they are further characterized by $R S$-stereoisomeric indices, $\left[\left[\mathbf{C}_{2 v}^{\prime}, \widetilde{\mathbf{C}}_{2 v}^{\prime}, \mathbf{C}_{2 \widehat{I}}\right]\right],\left[\left[\mathbf{C}_{2 v}^{\prime \prime}, \widetilde{\mathbf{C}}_{2 v}^{\prime \prime}, \mathbf{C}_{2 \widehat{I}}^{\prime}\right]\right]$, and $\left[\left[\mathbf{D}_{3 d}, \widetilde{\mathbf{D}}_{3 d}, \mathbf{D}_{3 \widehat{I}}\right]\right]$, respectively, in which the point-group symmetry $\left(\in \mathbf{O}_{h}\right)$, the $R S$-stereogenic symmetry $\left(\in \mathbf{O}_{\tilde{\sigma}}\right)$, and the $L R$ permutation symmetry $\left(\in \mathbf{O}_{\widehat{I}}\right)$ are collectively shown in a pair of double square brackets.

\subsubsection{Derivatives with composition $\mathrm{H}_{6} \mathrm{p}_{2}\left(\mathrm{H}_{6} \overline{\mathrm{p}}_{2}\right)$}

The $[6,0,0,0,0,0,2,0,0,0]^{*}$-row of Table 1 indicates the presence of the value $1(=1 / 2$ $\times 2$ ) at the $\mathbf{C}_{2}$-column (the 2 nd column), the $\mathbf{C}_{2}^{\prime}$-column (the 3 rd column), and the $\mathbf{D}_{3^{-}}$ column (the 17th column) under the point group $\mathbf{O}_{h}$. They are depicted as $\mathbf{1 0}\left(\mathbf{C}_{2}\right)$, $12\left(\mathbf{C}_{2}^{\prime}\right)$, and $14\left(\mathbf{D}_{3}\right)$ in the type-II sterereoisograms of Figure 7. Because one pair of enantiomers is counted once under the point group $\mathbf{O}_{h}$, they are depicted along the vertical direction, $\left(\left[\begin{array}{ll}\mathbf{1 0} & \overline{\mathbf{1 0}}\end{array}\right]\left(=\left[\begin{array}{ll}\mathbf{1 1} & \overline{\mathbf{1 1}}\end{array}\right]\right)\right)_{\mathrm{II}},\left(\left[\begin{array}{ll}\mathbf{1 2} & \overline{\mathbf{1 2}}\end{array}\right]\left(=\left[\begin{array}{ll}\mathbf{1 3} & \overline{\mathbf{1 3}}\end{array}\right]\right)\right)_{\mathrm{II}}$, and $\left(\left[\begin{array}{ll}\mathbf{1 4} & \overline{\mathbf{1 4}}\end{array}\right]\left(=\left[\begin{array}{ll}\mathbf{1 5} & \overline{\mathbf{1 5}}\end{array}\right]\right)\right)_{\mathrm{II}}$. In this enumeration, the first pair of enantiomers (e.g., $\left[\begin{array}{ll}\mathbf{1 0} & \mathbf{1 0}\end{array}\right]$ ), which is linked by a vertical double-headed arrow with an encircled bullet $(\longrightarrow \bullet)$, is degenerated with the second pair of enantiomers (e.g., $\left[\begin{array}{ll}\mathbf{1 1} & \overline{\mathbf{1 1}}\end{array}\right]$ ), so that the first pair is selected to give a selected pair of enantiomers $\left(\right.$ e.g. $\left(\left[\begin{array}{ll}\mathbf{1 0} & \overline{\mathbf{1 0}}\end{array}\right]\right)$ II belonging to $\mathbf{C}_{2}\left(\in \mathbf{O}_{h}\right)$. Note that the promolecule $\mathbf{1 0}$ corresponds to the composition $\mathrm{H}_{6} \mathrm{p}_{2}$, while its enantiomer $\overline{\mathbf{1 0}}$ corresponds to the composition $\mathrm{H}_{6} \overline{\mathrm{P}}_{2}$. Similarly, $\left(\left[\begin{array}{ll}\mathbf{1 2} & \overline{\mathbf{1 2}}\end{array}\right]\right)$ II belonging to $\mathbf{C}_{2}^{\prime}\left(\in \mathbf{O}_{h}\right)$, and $\left(\left[\begin{array}{ll}\mathbf{1 4} & \overline{\mathbf{1 4}}\end{array}\right]\right)$ II belonging to $\mathbf{D}_{3}\left(\in \mathbf{O}_{h}\right)$ are selected under the point group $\mathbf{O}_{h}$.

The $[6,0,0,0,0,0,2,0,0,0]^{*}$-row of Table 3 of the previous gross-enumeration [26] reported the total number $3(=3 / 2 \times 2)$ of cubane derivatives of the composition $\mathrm{H}_{6} \mathrm{p}_{2}$ 


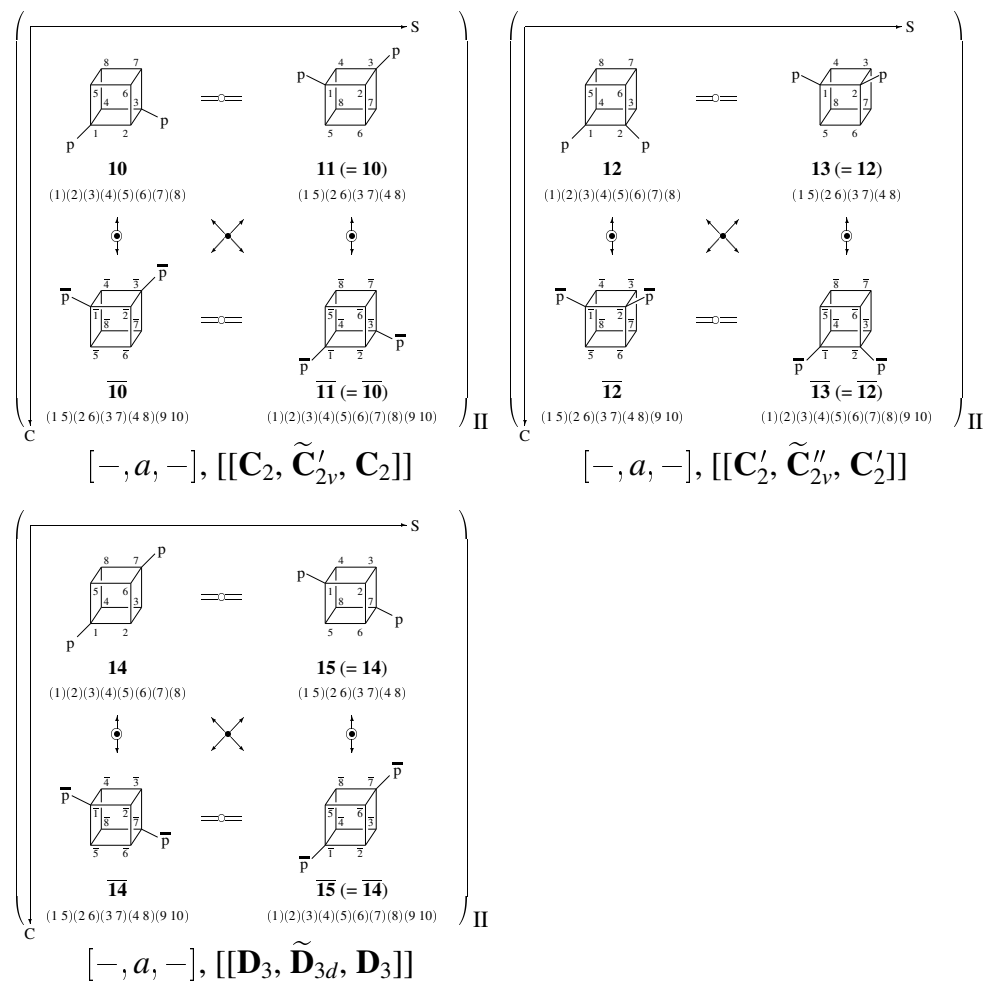

Figure 7. Stereoisograms of cubane derivative with the composition $\mathrm{H}_{6} \mathrm{p}_{2}\left(\mathrm{H}_{6} \overline{\mathrm{p}}_{2}\right)$. The type index $[-, a,-]$ is assigned to such a type-II stereoisogram, which is specified to be chiral, $R S$-astereogenic, and scleral.

$\left(\mathrm{H}_{6} \overline{\mathrm{p}}_{2}\right)$ under the point group $\mathbf{O}_{h}$ and the presence of $3(=3 / 2 \times 2)$ stereoisograms under the $R S$-stereoisomeric group $\mathbf{O}_{h \widetilde{\sigma} \widehat{I}}$. These gross values are consistent with the present symmetry-itemized enumeration.

The $[6,0,0,0,0,0,2,0,0,0]^{*}$-row of Table 4 indicates the apperance of the value 2 $(=1 \times 2)$ at the $\widetilde{\mathbf{C}}_{2 v}^{\prime}$-column (the 13th column), the $\widetilde{\mathbf{C}}_{2 v}^{\prime \prime}$-column (the 14 th column), and the $\widetilde{\mathbf{D}}_{3 d^{-}}$-column (the 28 th column) under the $R S$-stereogenic group $\mathbf{O}_{\tilde{\sigma}}$. They are depicted as $\mathbf{1 1}\left(\widetilde{\mathbf{C}}_{2 v}^{\prime}\right), \mathbf{1 3}\left(\widetilde{\mathbf{C}}_{2 v}^{\prime \prime}\right)$, and $\mathbf{1 5}\left(\widetilde{\mathbf{D}}_{3 d}\right)$ in the type-II sterereoisograms of Figure 7. Because one pair of self- $R S$-diastereomers is counted once under the $R S$-stereogenic group $\mathbf{O}_{\tilde{\sigma}}$, they are depicted along the horizontal direction, $([[\mathbf{1 0}(=\mathbf{1 1})]][[\overline{\mathbf{1 0}}(=\overline{\mathbf{1 1}})]])_{\mathrm{II}}$, $([[\mathbf{1 2}(=\mathbf{1 3})]][[\overline{\mathbf{1 2}}(=\overline{\mathbf{1 3}})]])_{\mathrm{II}}$, and $([[\mathbf{1 4}(=\mathbf{1 5})]][[\overline{\mathbf{1 4}}(=\overline{\mathbf{1 5}})]])_{\mathrm{II}}$, where each pair of self- 
$R S$-diastereomers (an $R S$-astereogenic promolecule) are surrounded by a pair of double square brackets. In this enumeration under $\mathbf{O}_{\widetilde{\sigma}}$, one pair of self- $R S$-diastereomers (an $R S$-astereogenic promolecule) is counted once. In each type-II stereoisogram in Figure 7, one pair of self- $R S$-diastereomers (an $R S$-astereogenic promolecule), e.g., ([[10 $=\mathbf{1 1})]]$, is linked by a horizontal equality symbol with a circle $(=0=)$. Another pair of self$R S$-diastereomers (an $R S$-astereogenic promolecule), e.g., $[[\overline{\mathbf{1 0}}(=\overline{\mathbf{1 1}})]])_{\mathrm{II}}$, is linked by a horizontal equality symbol with a circle $(=0)$. These two pairs of self- $R S$-diastereomers (two $R S$-astereogenic promolecules) are counted separately to give the value 2 under the $R S$-stereogenic group $\mathbf{O}_{\tilde{\sigma}}$.

The $[6,0,0,0,0,0,2,0,0,0]^{*}$-row of Table 7 indicates the appearance of the value $1(=$ $1 / 2 \times 2$ ) at the $\mathbf{C}_{2}$-column (the 2 nd column), the $\mathbf{C}_{2}^{\prime}$-column (the 3 rd column), and the $\mathbf{D}_{3}$-column (the 18th column) under the $L R$-permutation group $\mathbf{O}_{\widehat{I}}$. They are depicted as $\overline{\mathbf{1 1}}\left(\mathbf{C}_{2}\right), \overline{\mathbf{1 3}}\left(\mathbf{C}_{2}^{\prime}\right)$, and $\overline{\mathbf{1 5}}\left(\mathbf{D}_{3}\right)$ in the type-II sterereoisograms of Figure 7 . Because one pair of holantimers is counted once under the $L R$-permutation group $\mathbf{O}_{\widehat{I}}$, they are depicted along the diagonal direction, i.e., $\left(<\left[\begin{array}{ll}10 & \overline{\mathbf{1 1}}\end{array}\right]>\right)_{\mathrm{II}},\left(<\left[\begin{array}{ll}\mathbf{1 2} & \overline{\mathbf{1 3}}\end{array}\right]>\right)_{\mathrm{II}}$, and $\left(<\left[\begin{array}{ll}\mathbf{1 4} & \mathbf{1 5}\end{array}\right]\right)_{\mathrm{II}}$, each of which is counted once as a one pair of holantimers $(<[\ldots]>)$. In this enumeration under $\mathbf{O}_{\widehat{I}}$, one pair of holantimers is linked by a diagonal double-headed

arrow with a bullet $(\longrightarrow)$ in each type-II stereoisogram. Note that $\left(<\left[\begin{array}{ll}\mathbf{1 0} & \overline{\mathbf{1 1}}\end{array}\right]>\right)_{\text {II }}$ is selected to be counted, so as to nullify $\left(<\left[\begin{array}{ll}\mathbf{1 1} & \overline{\mathbf{1 0}}\end{array}\right]>\right)_{\text {II }}$ because of the presence of horizontal equality symbols $(\mathbf{1 0}=0=\mathbf{1 1}$ and $\overline{\mathbf{1 1}}=0=\overline{\mathbf{1 0}})$.

Each type-II stereoisograms depicted in Figure 7 is characterized by the type index $[-, a,-]$, which means chiral, $R S$-astereogenic, and scleral. By the present symmetryitemized enumeration, they are further characterized by $R S$-stereoisomeric indices, $\left[\left[\mathbf{C}_{2}\right.\right.$, $\left.\left.\widetilde{\mathbf{C}}_{2 v}^{\prime}, \mathbf{C}_{2}\right]\right],\left[\left[\mathbf{C}_{2}^{\prime}, \widetilde{\mathbf{C}}_{2 v}^{\prime \prime}, \mathbf{C}_{2}^{\prime}\right]\right]$, and $\left[\left[\mathbf{D}_{3}, \widetilde{\mathbf{D}}_{3 d}, \mathbf{D}_{3}\right]\right]$, in which the respective point-group symmetry $\left(\mathbf{C}_{2}, \mathbf{C}_{2}^{\prime}\right.$, or $\left.\mathbf{D}_{3}\left(\in \mathbf{O}_{h}\right)\right)$, the respective $R S$-stereogenic symmetry $\left(\widetilde{\mathbf{C}}_{2 v}^{\prime}, \widetilde{\mathbf{C}}_{2 v}^{\prime \prime}\right.$, or $\left.\widetilde{\mathbf{D}}_{3 d}\left(\in \mathbf{O}_{\widetilde{\sigma}}\right)\right)$, and the respective $L R$-permutation symmetry $\left(\mathbf{C}_{2}, \mathbf{C}_{2}^{\prime}\right.$, or $\left.\mathbf{D}_{3}\left(\in \mathbf{O}_{\widehat{I}}\right)\right)$ are collectively shown in a pair of double square brackets.

\subsection{Trisubstituted cubane derivatives}

\subsubsection{Derivatives with composition $\mathrm{H}_{5} \mathrm{ABX}$}

The $[5,1,1,1,0,0,0,0,0,0]$-row of Table 1 indicates the presence of the value 4 at the $\mathbf{C}_{1}$-column (the 1st column) and the value 6 at the $\mathbf{C}_{s}^{\prime}$-column (the 5 th column) under 
the point group $\mathbf{O}_{h}$.

Each of the four pairs of enantiomeric $\mathbf{C}_{1}$-derivatives belongs to a type-I stereoisogram shown in Figure 8, so that the value 4 at the $\mathbf{C}_{1}$-column means the appearance of four stereoisograms shown in Figure 8. The enantiomeric pair $\mathbf{1 6} / \overline{\mathbf{1 6}}$ is homomeric with the other enantiomeric pair $\mathbf{1 7} / \overline{\mathbf{1 7}}$ because of asclerality of the type-I stereoisogram $([-,-, a])$. Because of this type of homomerism, the four type-I stereoisograms are represented by $\left(\left[\begin{array}{ll}\mathbf{1 6} & \overline{\mathbf{1 6}}\end{array}\right)_{\mathrm{I}},\left(\left[\begin{array}{ll}\mathbf{1 8} & \overline{\mathbf{1 8}}\end{array}\right)_{\mathrm{I}},\left(\left[\begin{array}{ll}20 & \overline{\mathbf{2 0}}\end{array}\right)_{\mathrm{I}}\right.\right.\right.$, and $\left(\left[\begin{array}{ll}\mathbf{2 2} & \overline{\mathbf{2 2}}\end{array}\right)_{\mathrm{I}}\right.$ in a simplified manner.

In addition, the value 6 at the $\mathbf{C}_{s}^{\prime}$-column (the 5 th column) under the point group $\mathbf{O}_{h}$, which appear in the $[5,1,1,1,0,0,0,0,0,0]$-row of Table 1 , indicates the presence of six $\mathbf{C}_{s}^{\prime}$ cubane derivatives shown in Figure 9. They are achiral (belonging to the point group $\left.\mathbf{C}_{s}^{\prime}\left(\in \mathbf{O}_{h}\right)\right)$ and designated by the type-IV stereoisogram symbols: $([\mathbf{2 4}])_{\mathrm{IV}},([\mathbf{2 6}])_{\mathrm{IV}}$, $([\mathbf{2 8}])_{\mathrm{IV}},([\mathbf{3 0}])_{\mathrm{IV}},([\mathbf{3 2}])_{\mathrm{IV}}$, and $([\mathbf{3 4}])_{\mathrm{IV}}$.

The $[5,1,1,1,0,0,0,0,0,0]$-row of Table 3 of the previous gross-enumeration [26] reported the total number 10 of cubane derivatives of the composition $\mathrm{H}_{6} \mathrm{ABX}$ under the point group $\mathbf{O}_{h}$ and the presence of 10 stereoisograms under the $R S$-stereoisomeric group $\mathbf{O}_{h \widetilde{\sigma} \widehat{I}}$. These gross values are consistent with the present symmetry-itemized enumeration summarized in Figures 8 and 9.

The $[5,1,1,1,0,0,0,0,0,0]$-row of Table 4 indicates the presence of the value 4 at the $\mathbf{C}_{1}$-column (the 1st column) and the value 6 at the $\widetilde{\mathbf{C}}_{s}^{\prime}$-column (the 5 th column) under the $R S$-stereogenic group $\mathbf{O}_{\widetilde{\sigma}}$.

As a result, the four type-I stereoisograms shown in Figure 8 are alternatively examined by the action of the $R S$-stereogenic group $\mathbf{O}_{\tilde{\sigma}}$. For example, the $\mathbf{C}_{1}$-pair of $R S$-diastereomers $\mathbf{1 6} / \mathbf{1 7}$ is homomeric with the other $\mathbf{C}_{1}$-pair of $R S$-diastereomers $\overline{\mathbf{1 7}} / \overline{\mathbf{1 6}}$ because of asclerality of the type-I stereoisogram $([-,-, a])$. Because of this type of homomerism, there appear four simplified $\mathrm{C}_{1}$-pair of $R S$-diastereomers, $\left(\left[\begin{array}{ll}16 & 17\end{array}\right)_{\mathrm{I}},\left(\left[\begin{array}{ll}\mathbf{1 8} & \mathbf{1 9}\end{array}\right]\right)_{\mathrm{I}}\right.$, $\left(\begin{array}{ll}20 & 21\end{array}\right)_{\mathrm{I}}$, and $\left(\left[\begin{array}{ll}22 & 23\end{array}\right)_{\mathrm{I}}\right.$, which are selected under the action of the $R S$-stereogenic group $\mathbf{O}_{\widetilde{\sigma}}$. This set can be equalified to the set of $\left(\left[\begin{array}{ll}\mathbf{1 6} & \overline{\mathbf{1 6}}\end{array}\right)_{\mathrm{I}},\left(\left[\begin{array}{ll}\mathbf{1 8} & \overline{\mathbf{1 8}}\end{array}\right)_{\mathrm{I}},\left(\left[\begin{array}{ll}\mathbf{2 0} & \overline{\mathbf{2 0}}\end{array}\right)_{\mathrm{I}}\right.\right.\right.$, and $\left(\left[\begin{array}{ll}22 & \overline{22}\end{array}\right]\right)$, which are obtained under the point group $\mathbf{O}_{h}$.

In addition, the value 6 at the $\widetilde{\mathbf{C}}_{s}^{\prime}$-column (the 5 th column) under the $R S$-stereogenic group $\mathbf{O}_{\widetilde{\sigma}}$, which appear in the $[5,1,1,1,0,0,0,0,0,0]$-row of Table 4, indicates the presence of six $\widetilde{\mathbf{C}}_{s}^{\prime}$-cubane derivatives shown in Figure 9. For example, the derivative $\mathbf{2 4}$ in the first type-IV stereoisogram belongs to the $R S$-stereogenic group $\widetilde{\mathbf{C}}_{s}^{\prime}$, which is a subgroup 

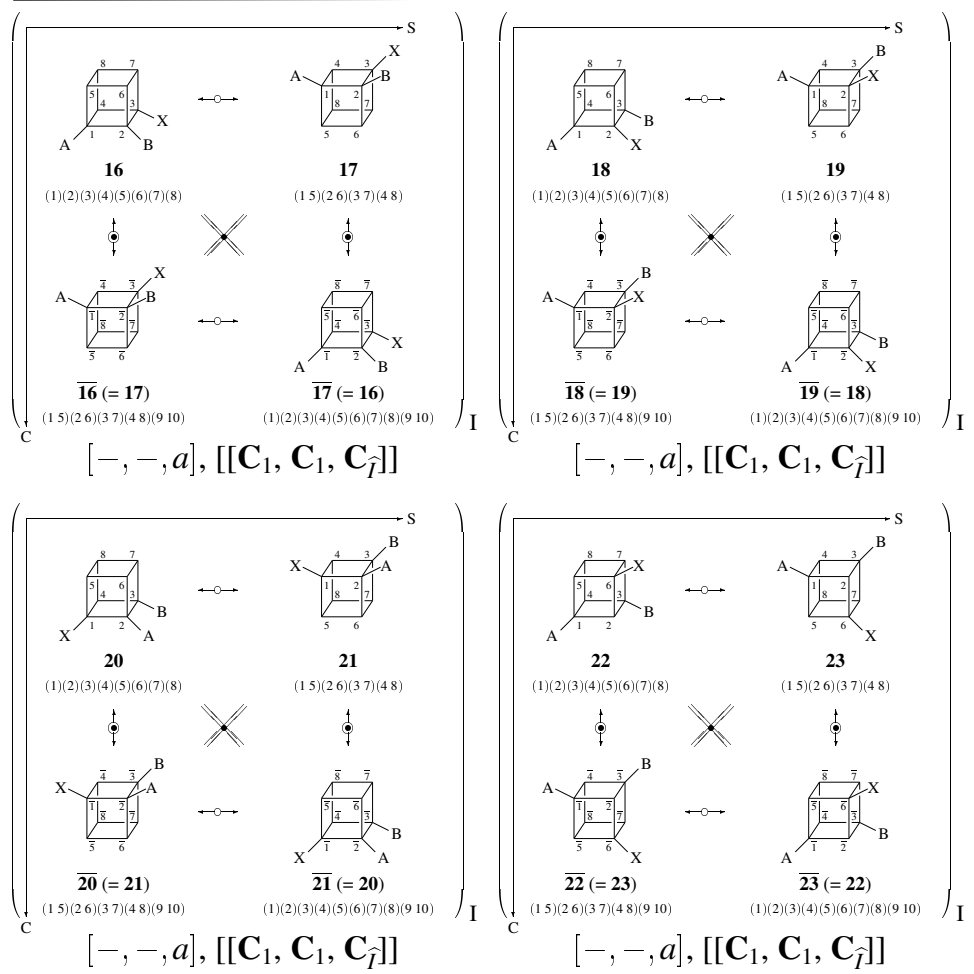

Figure 8. Four type-I stereoisograms of cubane derivative with the composition $\mathrm{H}_{5} \mathrm{ABX}$. The type index $[-,-, a]$ is assigned to such a type-I stereoisogram, which is specified to be chiral, $R S$-stereogenic, and ascleral.

of the $R S$-stereogenic group $\mathbf{O}_{\widetilde{\sigma}}$.

The $[5,1,1,1,0,0,0,0,0,0]$-row of Table 7 indicates the presence of the value 14 at the $\mathbf{C}_{\widehat{I}^{-}}$colum (the 4 th column) under the $L R$-permutation group $\mathbf{O}_{\widehat{I}}$.

Because one pair of (self-)holantimers is counted once under the $L R$-permutation group $\mathbf{O}_{\widehat{I}}$, the two promolecues along the diagonal direction are coupled to give a pair of holantimers or self-holantimers. For example, the set of two self-holantimeric pairs $(<\mathbf{1 6} \overline{\mathbf{1 7}}><\mathbf{1 7} \overline{\mathbf{1 6}}>)_{\text {I }}$ in the first type-I stereoisogram of Figure 8 are counted separately under $\mathbf{O}_{\widehat{I}}$. Thus, the self-holantimeric pair $<\mathbf{1 6} \overline{\mathbf{1 7}}>$ (an ascleral promolecule belonging to $\mathrm{C}_{\widehat{I}}$ ) and the other self-holantimeric pair $<\mathbf{1 7} \overline{\mathbf{1 6}}>$ (an ascleral promolecule belonging to $\mathbf{C}_{\widehat{I}}$ ) are not superimposable under the $L R$-permutation group $\mathbf{O}_{\widehat{I}}$. It follows 

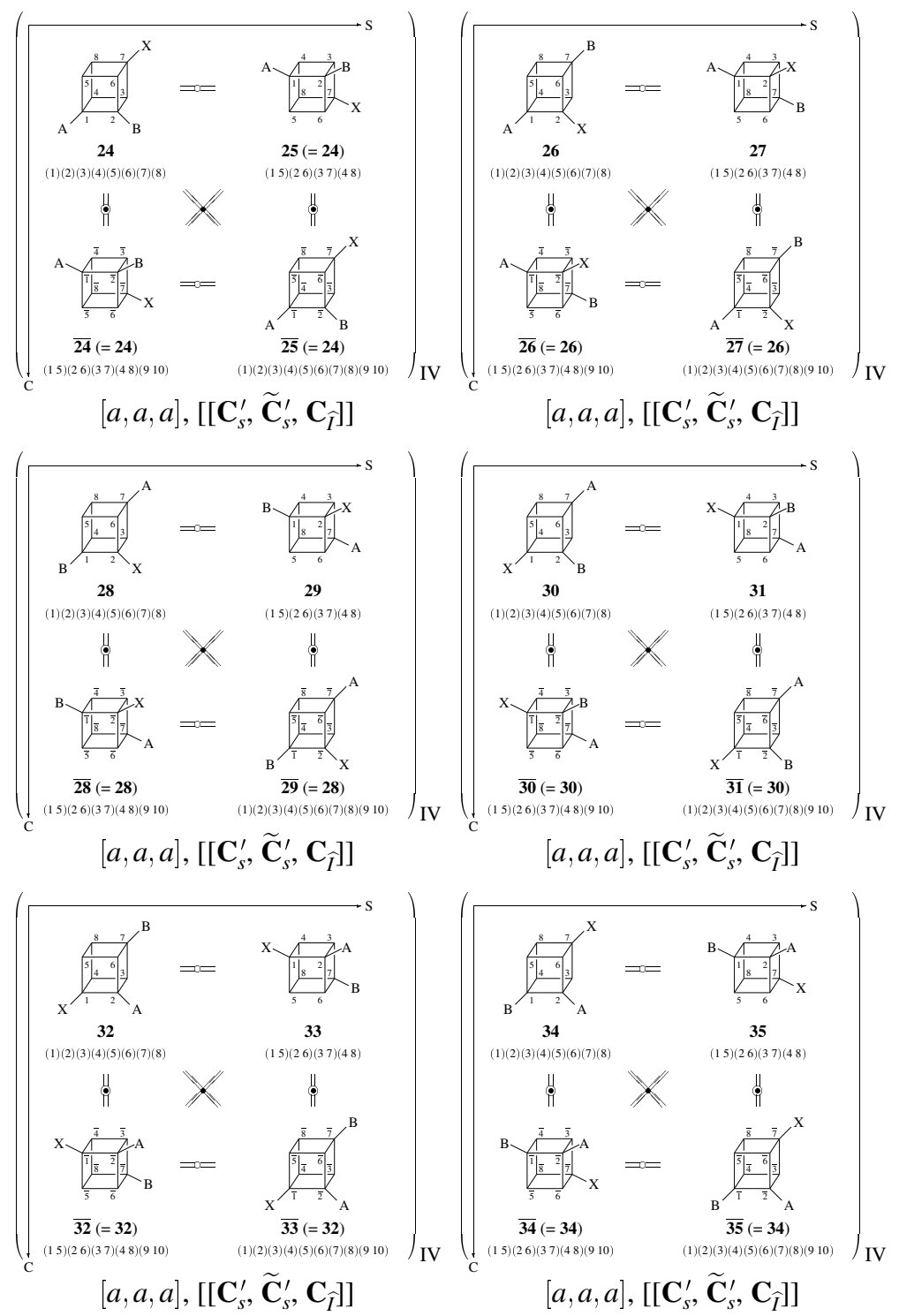

Figure 9. Six type-IV stereoisograms of cubane derivative with the composition $\mathrm{H}_{5} \mathrm{ABX}$. The type index $[a, a, a]$ is assigned to such a type-IV stereoisogram, which is specified to be achiral, $R S$-astereogenic, and ascleral. 
that two ascleral promolecules $<\mathbf{1 6} \overline{\mathbf{1 7}}>$ and $<\mathbf{1 7} \overline{\mathbf{1 6}}>$ are counted separately to give a vaule 2 under the $L R$-permutation group $\mathbf{O}_{\widehat{I}}$. The remaining type-I stereoisograms can be examined in a similar way to give the three sets: $(<\mathbf{1 8} \overline{\mathbf{1 9}}><\mathbf{1 9} \overline{\mathbf{1 8}}>)_{\mathrm{I}}$, $(<20 \overline{21}><21 \overline{20}>)_{\text {I }},(<22 \overline{\mathbf{2 3}}><23 \overline{\mathbf{2 2}}>)_{\text {I }}$, Totally, the value $8(=2 \times$ 4) are estimated due to the four type-I stereoisograms of Figure 8 and contribute to the value 14 under the $L R$-permuation group $\mathbf{O}_{\widehat{I}}$.

The remaining value $6(=14-8)$ among the value 14 in the enumeration under the $L R$-permutation group $\mathbf{O}_{\widehat{I}}$ can be explained by six type-IV stereoisograms shown in Figure 9 .

In the right of the above discussions, each type-I stereoisogram of Figure 8 is characterized a type index and the type index $[-,-, a]$ and the $R S$-stereoisomeric index $\left[\left[\mathbf{C}_{1}, \mathbf{C}_{1}\right.\right.$, $\left.\mathbf{C}_{\widehat{I}}\right]$.

Each of the type-IV stereoisograms depicted in Figure 9 is characterized by the type index $[a, a, a]$, which means achiral, $R S$-astereogenic, and ascleral. By the present symmetry-itemized enumeration, each of them is further characterized by a $R S$-stereoisomeric index, $\left[\left[\mathbf{C}_{s}^{\prime}, \widetilde{\mathbf{C}}_{s}^{\prime}, \mathbf{C}_{\widehat{I}}\right]\right]$, in which the point-group symmetry $\mathbf{C}_{s}^{\prime}\left(\in \mathbf{O}_{h}\right)$, the $R S$-stereogenic symmetry $\widetilde{\mathbf{C}}_{s}^{\prime}\left(\in \mathbf{O}_{\widetilde{\sigma}}\right)$, and the $L R$-permutation symmetry $\mathbf{C}_{\widehat{I}}\left(\in \mathbf{O}_{\widehat{I}}\right)$ are collectively shown in a pair of double square brackets.

\subsubsection{Derivatives with composition $\mathrm{H}_{5} \mathrm{Ap} \overline{\mathrm{p}}$}

Enumeration under the point group $\mathbf{O}_{h}$ is consistent with the previous enumeration [20], which was conducted by means of Maple programming system. The $[5,1,0,0,0,0,1,1,0,0]$ row of Table 1 indicates the presence of the value 5 at the $\mathbf{C}_{1}$-column (the first column) and the value 4 at the $\mathbf{C}_{s}^{\prime}$-column (the 5 th column). They are depicted in Figure 10 . Among them, the top three type-II stereoisograms are characterized by the presence of horizontal equality symbols. They are respectively composed of $\left(\left[\begin{array}{ll}\mathbf{3 6} & \overline{\mathbf{3 6}}\end{array}\right)_{\mathrm{II}},\left(\left[\begin{array}{ll}\mathbf{3 8} & \overline{\mathbf{3 8}}\end{array}\right)_{\mathrm{II}}\right.\right.$, and $\left(\left[\begin{array}{ll}40 & \overline{40}\end{array}\right)_{\mathrm{II}}\right.$, where each pair is a pair of enantiomers, which is counted once as a $\mathbf{C}_{1}$-derivative under the point group $\mathbf{O}_{h}$. In contrast, the type-III stereoisogram at the right side of the middle row of Figure 10 is characterized by the absence of equality symbols in all directions. This type-III stereoisogram is composed of two pairs of enantiomers, i.e., $\left(\left[\begin{array}{ll}42 & \overline{42}\end{array}\right]\left[\begin{array}{ll}43 & \overline{43}\end{array}\right)_{\text {III }}\right.$, and counted to give the value 2 as two pairs of $\mathbf{C}_{1}$-isomers. The sum value 3 from the three type-II stereoisograms and the value 2 from the one type-III stereoisogram totally explain the value 5 at the intersection between the 
$[5,1,0,0,0,0,1,1,0,0]$-row and the $\mathbf{C}_{1}$ column of Table 1.

The value 4 at the $\mathbf{C}_{s}^{\prime}$-column (the 5 th column) of the $[5,1,0,0,0,0,1,1,0,0]$-row of Table 1 is explained by the two type- $\mathrm{V}$ stereoisograms depicted at the bottom row of Figure 10. These type- $\mathrm{V}$ stereoisograms are characterized by the presence of vertical equality symbols. Each of the type-V stereoisograms contains two achiral cubane derivatives $([\mathbf{4 2}][43])_{\mathrm{V}}\left(\right.$ the bottom-left diagram) and $([\mathbf{4 4}][\mathbf{4 5}])_{\mathrm{V}}$ (the bottom-right diagram). Hence totally $4(=2 \times 2)$ achiral cubane derivatives are consistent with the data 4 at the intersection between the $[5,1,0,0,0,0,1,1,0,0]$-row and the 5 th $\mathbf{C}_{s}^{\prime}$-column of Table 1 .

The $[5,1,0,0,0,0,1,1,0,0]$-row of Table 4 indicates the presence of the value 4 at the $\mathbf{C}_{1}$-column (the 1st column) and the value 6 at the $\widetilde{\mathbf{C}}_{s}^{\prime}$-column (the 5 th column) under the $R S$-stereogenic group $\mathbf{O}_{\tilde{\sigma}}$.

The three type-II stereoisograms shown in Figure 10 are alternatively examined by the action of the $R S$-stereogenic group $\mathbf{O}_{\tilde{\sigma}}$. Because of the existence of horizontal equality symbols, the pair of self- $R S$-diastereomers (i.e. one $R S$-astereogenic promolecule) 36/37 (=36) is counted once under $\mathbf{O}_{\tilde{\sigma}}$; and at the same time, the other pair of self- $R S$ diastereomers (i.e. one $R S$-astereogenic promolecule) $\overline{\mathbf{3 6}} / \overline{\mathbf{3 7}}(=\overline{\mathbf{3 6}})$ is counted once under $\mathbf{O}_{\tilde{\sigma}}$. These two pairs in the top-left type-II stereoisogram of Figure 10 are counted separately under $\mathbf{O}_{\widetilde{\sigma}}$ (not under $\mathbf{O}_{h}$ ), so that one type-II stereoisogram contributes 2 among the value 6 at the 5 th column of the $[5,1,0,0,0,0,1,1,0,0]$-row of Table 4 . In a similar way, the pair of self- $R S$-diastereomers (i.e. one $R S$-astereogenic promolecule) $38 / 39$ (= 38 ) and the other pair of self- $R S$-diastereomers (i.e. one $R S$-astereogenic promolecule) $\overline{\mathbf{3 8}} / \overline{\mathbf{3 9}}(=\overline{\mathbf{3 8}})$ are counted separately in the top-right type-II stereoisogram of Figure 10; as well as the pair of self- $R S$-diastereomers (i.e. one $R S$-astereogenic promolecule) $\mathbf{4 0 / 4 1}$ $(=40)$ and the other pair of self- $R S$-diastereomers (i.e. one $R S$-astereogenic promolecule) $\overline{\mathbf{4 0}} / \overline{\mathbf{4 1}}(=\overline{\mathbf{4 0}})$ are counted separately in the middel-left type-II stereoisogram of Figure 10. Hence, the three type-II stereoisograms totally contribute $6(=2 \times 3)$ so as to be consistent with the enumeration data.

The value 4 at the intersection between the $[5,1,0,0,0,0,1,1,0,0]$-row and the $\mathbf{C}_{1^{-}}$ column (the 1st column) of Table 4 can be explained by the type-III stereoisogram (the middle-right diagram in Figure 10) and the two type-V stereoisograms (the bottom two diagrams in Figure 10). The one pair of $R S$-diastereomers 42/43 and the other pair of $R S$-diastereomers $\overline{\mathbf{4 2}} / \overline{\mathbf{4 3}}$ are counted separately so as to contribute 2 among the value 4 . 

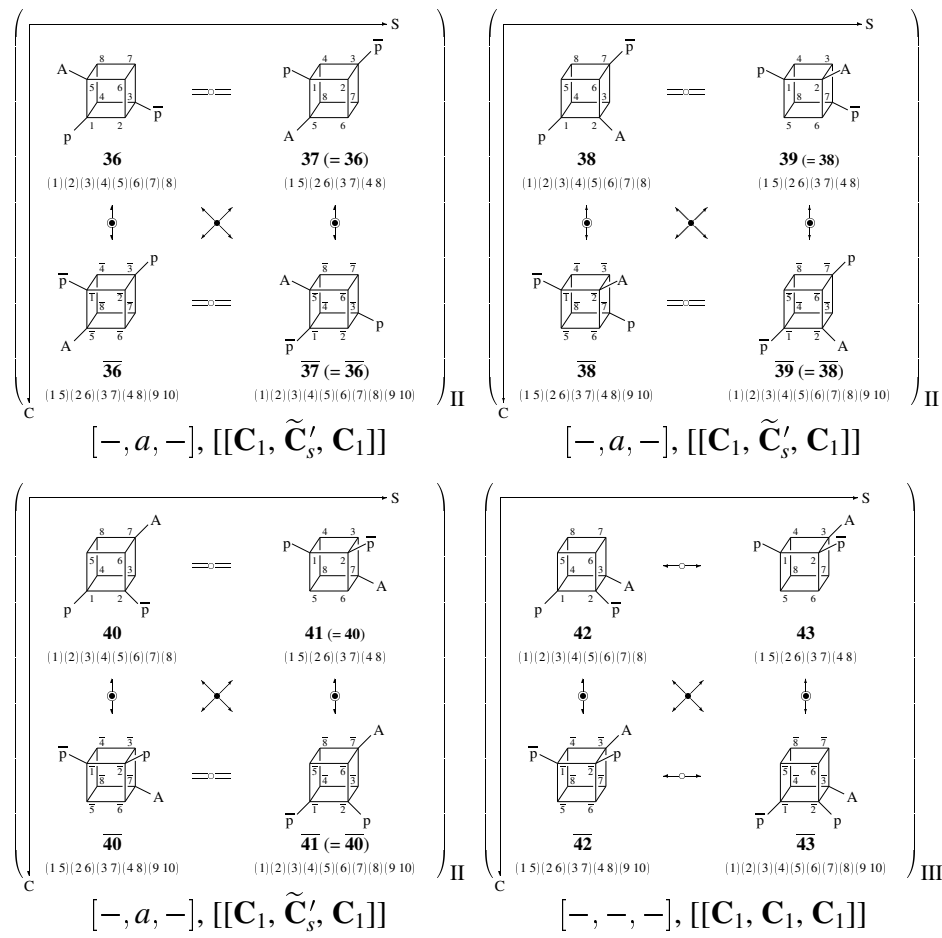

$[-, a,-],\left[\left[\mathbf{C}_{1}, \widetilde{\mathbf{C}}_{s}^{\prime}, \mathbf{C}_{1}\right]\right]$
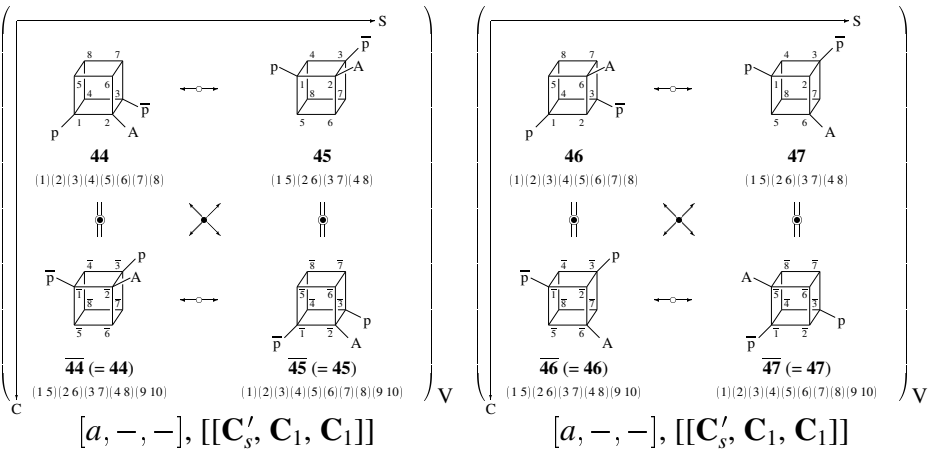

Figure 10. Stereoisograms of cubane derivatives with the composition $\mathrm{H}_{5} \mathrm{Ap} \overline{\mathrm{p}}$. The type index $[-, a,-]$ is assigned to such a type-II stereoisogram, which is specified to be achiral, $R S$-astereogenic, and ascleral. The type index $[-,-,-]$ is assigned to such a type-III stereoisogram, which is specified to be chiral, $R S$-stereogenic, and scleral. The type index $[a,-,-]$ is assigned to such a type- $\mathrm{V}$ stereoisogram, which is specified to be achiral, $R S$-stereogenic, and scleral. 
On the other hand, $([44][45])_{\mathrm{V}}$ is counted once as a pair of $R S$-diastereomers under $\mathbf{O}_{\widetilde{\sigma}}$ as well as $([\mathbf{4 6}][\mathbf{4 7}])_{\mathrm{V}}$ is separately counted once as another pair of $R S$-diastereomers under $\mathbf{O}_{\tilde{\sigma}}$. Hence, the two type- $\mathrm{V}$ stereoisograms contribute 2 among the value 4 . As a result, the type III stereoisogram and the two type-V stereoisograms totally contribute 4 $(=2+1 \times 2)$.

The $[5,1,0,0,0,0,1,1,0,0]$-row of Table 7 indicates the presence of the value 7 at the $\mathbf{C}_{1}$-column (the 1st column) under the $L R$-permutation group $\mathbf{O}_{\widehat{I}}$.

Under the $L R$-permutation group $\mathbf{O}_{\widehat{I}}$, all stereisograms shown in Figure 10 have diagonal double-headed arrows, which means that they are scleral to be concerned with pairs of holantimers. Thus, one pair of holantimers is counted once in each type-II stereoisograms (totally $1 \times 3=3$ ), two pairs of holantimers are counted to give 2 in type-III stereisograms (totally $2 \times 1=2$ ), and one pair of holantimers is counted once in each type- $\mathrm{V}$ stereoisogram (totally $1 \times 2=2$ ). As a result, we have the total value, as to be $3+2+2=7$.

In the light of the above discussions, each type-II stereoisogram of Figure 10 is characterized by a type index $[-, a,-]$ and by the corresponding $R S$-stereoisomeric index $\left[\left[\mathbf{C}_{1}\right.\right.$, $\left.\widetilde{\mathbf{C}}_{s}^{\prime}, \mathbf{C}_{1}\right]$; the type-III stereoisogram of Figure 10 is characterized by a type index $[-,-,-]$, and the corresponding $R S$-stereoisomeric index $\left[\left[\mathbf{C}_{1}, \mathbf{C}_{1}, \mathbf{C}_{1}\right]\right]$; and each type-V stereoisogram of Figure 10 is characterized by a type index $[a,-,-]$, and the corresponding $R S$-stereoisomeric index $\left[\left[\mathbf{C}_{s}^{\prime}, \widetilde{\mathbf{C}}_{s}^{\prime}, \mathbf{C}_{1}\right]\right]$.

The above type-V stereoisograms, i.e., $([\mathbf{4 4}][4 \mathbf{4 5}])_{\mathrm{V}}$ and $([\mathbf{4 6}][\mathbf{4 7}])_{\mathrm{V}}$, should be discussed in details, because they are futher examples of an extended pseudoasymmetry [41]. Thus, the two achiral cubane derivatives [44] and [45] with the composition $\mathrm{H}_{5} \mathrm{Ap} \overline{\mathrm{p}}$, which are contained in the type-V stereoisogram $([\mathbf{4 4}] \quad[\mathbf{4 5}])_{\mathrm{V}}$, are $R S$-diastereomeric to each other under the $R S$-stereogenic group $\mathbf{O}_{\tilde{\sigma}}$. Similarly, the two achiral cubane derivatives $[\mathbf{4 6}]$ and $[\mathbf{4 7}]$ with the composition $\mathrm{H}_{5} \mathrm{Ap} \overline{\mathrm{p}}$, which are contained in the other type-V stereoisogram $([\mathbf{4 6}][\mathbf{4 7}])_{\mathrm{V}}$, are $R S$-diastereomeric to each other under the $R S$ stereogenic group $\mathbf{O}_{\widetilde{\sigma}}$. For the assignment of $C / A$-descriptors, the code $C U$-8-14244434- $a$ is assigned to 44, while the code $C U-8-14244434-c$ is assigned to 45. The lowercase letters $a$ and $c$ are $C / A$-descriptors for specifying chirality-unfaithful features between 44 and $45[26]$.

In contrast, the $[6,0,0,0,0,0,1,1,0,0]$-row of Table 1 shows the presence of one $\mathbf{C}_{s^{-}}$, 
one $\mathbf{C}_{s^{-}}^{\prime}$, and one $\mathbf{C}_{3 i}$-promolecules with the composition $\mathrm{H}_{6} \mathrm{p} \overline{\mathrm{p}}$, which are found to be all achiral and to belong to type-IV stereoisograms by referring to the corresponding rows of the composition $\mathrm{H}_{6} \mathrm{p} \overline{\mathrm{p}}$ in Tables 4 and 7 . Note that the proligand $\mathrm{A}$ is present in the composition $\mathrm{H}_{5} \mathrm{Ap} \overline{\mathrm{p}}$ (type- $\mathrm{V}$, extended pseudoasymmetry) but absent in the composition $\mathrm{H}_{6} \mathrm{p} \overline{\mathrm{p}}$ (type-IV). This situation is akin to the case of $\mathrm{H}_{2} \mathrm{p} \overline{\mathrm{p}}$ (type-IV) vs. HAp $\overline{\mathrm{p}}$ (type-V, pseudoasymmetry) appearing in tetrahedral carbons $[8,9]$. This parallelism reveals the importance of skeletons in the proligand-promolecule model $[42,43]$ proposed by us. Thus, the cubane skeleton and the tetrahedral skeleton can be discussed on the basis of common standpoints under the proligand-promolecule model both for Fujita's USCI approach [42] and Fujita's stereoisogram approach [43].

\section{Conclusive remarks}

Symmetry-itemized enumerations of cubane derivatives are conducted by considering three-aspects of symmetry, i.e., the point group $\mathbf{O}_{h}$ for chirality/achirality, the $R S$ stereogenic group $\mathbf{O}_{\tilde{\sigma}}$ for $R S$-stereogenicity/ $R S$-astereogenicity, and the $L R$-permutation group $\mathbf{O}_{\widehat{I}}$ for sclerality/ascrelarity, where these groups are considered as subgroups of $R S$ stereoisomeric group $\mathbf{O}_{h \widetilde{\sigma} \widehat{I}}$. Each group is generated by using a set of generators under the GAP (Groups, Algorithms and Programming) system, where a combined-permutation representation $(\mathrm{CPR})$ is proposed as a new expression of reflection operations. Stereoisograms are adopted as diagramatical expressions of $R S$-stereoisomeric group $\mathbf{O}_{h \widetilde{\sigma} \widehat{I}}$. The partial-cycle-index (PCI) method of Fujita's unit-subduced-cycle-index (USCI) approach is adopted in symmetry-itemized enumerations due to Fujita's stereoisogram approach. Five types of stereoisograms are used to explain the data of symmetry-itemized enumerations after the type indices for stereoisograms (e.g., $[a,-,-]$ for a type-V stereoisogram) is sophisticated into $R S$-stereoisomeric indices (e.g., $\left[\left[\mathbf{C}_{s}^{\prime}, \widetilde{\mathbf{C}}_{s}^{\prime}, \mathbf{C}_{1}\right]\right]$ for a cubane derivative with the composition $\left.\mathrm{H}_{5} \mathrm{Ap} \overline{\mathrm{p}}\right)$. The type-V stereoisograms for cubane derivatives with the composition $\mathrm{H}_{5} \mathrm{Ap} \overline{\mathrm{p}}$ are discussed under extended pseudoasymmetry as a new concept.

\section{References}

[1] J. H. van't Hoff, Sur les formules de structure dans l'espace, Archives Néerlandaises des Sciences exactes et naturelles 9 (1874) 445-454.

[2] J. H. van't Hoff, A suggestion looking to the extension into space of the structural formulas at present used in chemistry. And a note upon the relation between the optical 
activity and the chemical constitution of organic compounds in: G. M. Richardson (Ed.), Foundations of Stereochemistry, Memoirs of Pasteur, van't Hoff, Le Bel and Wislicenus, American Book, New York, 1901, pp. 35-46.

[3] J. A. Le Bel, Sur les relations qui existent entre les formules atomiques des corps organique et le pouvoir rotatoire de leurs dissolutions, Bull. Soc. Chim. Fr. (2) 22 (1874) 337-347.

[4] J. A. Le Bel, On the relations which exist between the atomic formulas of organic compounds and the rotatory power of their solutions, in: O. T. Benfey (Ed.), Classics in the Theory of Chemical Combination, Dover, New York, 1963, pp 161-171.

[5] S. Fujita, Conceptual defects of modern stereochemistry. Comprehensive remedy by stereoisograms based on $R S$-stereoisomerism for mediating between enantiomerism and stereoisomerism, Tetrahedron: Asymmetry 28 (2017) 1-33.

[6] K. Mislow, J. Siegel, Stereoisomerism and local chirality, J. Am. Chem. Soc. 106 (1984) 3319-3328.

[7] W. T. Kelvin, The Baltimore Lectures on Molecular Dynamics and the Wave Theory of Light, Clay \& Sons, London, 1904, pp. 619-619.

[8] S. Fujita, Stereogenicity revisited. Proposal of holantimers for comprehending the relationship between stereogenicity and chirality, J. Org. Chem. 69 (2004) 31583165

[9] S. Fujita, Pseudoasymmetry, stereogenicity, and the $R S$-nomenclature comprehended by the concepts of holantimers and stereoisograms, Tetrahedron 60 (2004) 1162911638.

[10] S. Fujita, Chirality and $R S$-stereogenicity as two kinds of handedness. Their Aufheben by Fujita's stereoisogram approach for giving new insights into classification of isomers, Bull. Chem. Soc. Jpn. 89 (2016) 987-1017.

[11] S. Fujita, Integrated discussion on stereogenicity and chirality for restructuring stereochemisty, J. Math. Chem. 35 (2004) 265-287.

[12] S. Fujita, Combinatorial Enumeration of Graphs, Three-Dimensional Structures, and Chemical Compounds, Univ. Kragujevac, Kragujevac, 2013.

[13] S. Fujita, Combinatorial enumeration of cubane derivatives as three-dimensional entities. I. Gross enumeration by the proligand method, MATCH Commun. Math. Comput. Chem. 67 (2012) 5-24.

[14] S. Fujita, Combinatorial enumeration of cubane derivatives as three-dimensional entities. II. Gross enumeration by the markaracter method, MATCH Commun. Math. Comput. Chem. 67 (2012) 25-54.

[15] S. Fujita, Combinatorial enumeration of cubane derivatives as three-dimensional entities. III. Gross enumeration by the characteristic-monomial method, MATCH Commun. Math. Comput. Chem. 67 (2012) 649-668. 
[16] S. Fujita, Combinatorial enumeration of cubane derivatives as three-dimensional entities. IV. Gross enumeration by the extended superposition method, MATCH Commun. Math. Comput. Chem. 67 (2012) 669-686.

[17] S. Fujita, Combinatorial enumeration of cubane derivatives as three-dimensional entities. V. Gross enumeration by the double coset representation method, MATCH Commun. Math. Comput. Chem. 67 (2012) 687-712.

[18] S. Fujita, Symmetry and Combinatorial Enumeration in Chemistry, Springer-Verlag, Berlin, 1991.

[19] S. Fujita, Diagrammatical Approach to Molecular Symmetry and Enumeration of Stereoisomers, Univ. Kragujevac, Kragujevac, 2007.

[20] S. Fujita, Symmetry-itemized enumeration of cubane derivatives as three-dimensional entities by the partial-cycle-index method of the USCI approach, Bull. Chem. Soc. Jpn. 85 (2012) 793-810.

[21] S. Fujita, Symmetry-itemized enumeration of cubane derivatives as three-dimensional entities by the elementary-superposition method of the USCI approach, Bull. Chem. Soc. Jpn. 85 (2012) 811-821.

[22] S. Fujita, Cage-shaped molecules derived by applying the edge strategy to a cubane skeleton., J. Comput. Chem. Jpn. Int. Ed. 1 (2015) 1-4.

[23] S. Fujita, Systematic enumeration and symmetries of cubane derivatives, Chem. Rec. 16 (2016) 1116-1163.

[24] S. Fujita Stereoisograms: A remedy against oversimplified dichotomy between enantiomers and diastereomers in stereochemistry, in: M. V. Putz (Ed.), Chemical Information and Computational Challenge in the 21st Chentury, Nova, New York, 2012, pp. 223-242.

[25] S. Fujita, Mathematical Stereochemistry, De Gruyter, Berlin, 2015.

[26] S. Fujita, Stereoisograms of cubane derivatives., Bull. Chem. Soc. Jpn. 88 (2015) 1653-1679.

[27] S. Fujita, Computer-oriented representations of point groups and cycle indices with chirality fittingness (CI-CFs) calculated by the GAP system. Enumeration of threedimensional structures of ligancy 4 by Fujita's proligand method, MATCH Commun. Math. Comput. Chem. 76 (2016) 379-400.

[28] https://www.gap-system.org/.

[29] S. Fujita, Concordant generation of mark tables and USCI-CF (unit subduced cycle indices with chirality fittingness) tables on the basis of combined-permutation representations, MATCH Commun. Math. Comput. Chem. 82 (2019) 295-326.

[30] S. Fujita, Standardization of mark tables and USCI-CF (unit subduced cycle indices with chirality fittingness) tables derived from different $\mathbf{O}_{h}$-skeletons, $M A T C H$ Commun. Math. Comput. Chem. 82 (2019) 327-373. 
[31] S. Fujita, Promolecules with a subsymmetry of $\mathbf{O}_{h}$. Combinatorial enumeration and stereochemical properties, Polyhedron 12 (1993) 95-110.

[32] S. Fujita, Subduction of coset representations. An application to enumeration of chemical structures, Theor. Chim. Acta 76 (1989) 247-268.

[33] S. Fujita, Subduction of coset representations. An application to enumeration of chemical structures with achiral and chiral ligands, J. Math. Chem. 5 (1990) 121156.

[34] S. Fujita, Systematic enumeration of high symmetry molecules by means of unit subduced cycle indices with and without chirality fittingness, Bull. Chem. Soc. Jpn. 63 (1990) 203-215.

[35] S. Fujita, Enumeration of digraphs with a given automorphism group, J. Math. Chem. 12 (1993) 173-195.

[36] S. Fujita, Generalization of partial cycle indices and modified bisected mark tables for combinatorial enumeration, Bull. Chem. Soc. Jpn. 73 (2000) 329-339.

[37] S. Fujita, The USCI approach and elementary superposition for combinatorial enumeration, Theor. Chim. Acta 82 (1992) 473-498.

[38] S. Fujita, Graphs to chemical structures 1. Sphericity indices of cycles for stereochemical extension of Pólya's theorem, Theor. Chem. Acc. 113 (2005) 73-79.

[39] S. Fujita, Symmetry-itemized enumeration of cubane derivatives as three-dimensional entities by the fixed-point matrix method of the USCI approach, Bull. Chem. Soc. Jpn. 84 (2011) 1192-1207.

[40] S. Fujita, A proof for the existence of five stereogenicity types on the basis of the existence of five types of subgroups of $R S$-stereoisomeric groups. Hierarchy of groups for restructuring stereochemistry (Part 3), MATCH Commun. Math. Comput. Chem. 54 (2005) 39-52.

[41] S. Fujita, Extended pseudoasymmetry and geometric prochirality clarifying the scope of the concepts of holantimers and stereoisograms, Tetrahedron: Asymmetry $\mathbf{2 3}$ (2012) 623-634.

[42] S. Fujita, Importance of the proligand-promolecule model in stereochemistry. I. The unit-subduced-cycle-index (USCI) approach to geometric features of prismane derivatives, J. Math. Chem. 50 (2012) 2202-2222.

[43] S. Fujita, Importance of the proligand-promolecule model in stereochemistry. II. The stereoisogram approach to stereoisomeric features of prismane derivatives, J. Math. Chem. 50 (2012) 2168-2201. 


\section{Appendix A}

\#Read("c:/fujita000/fujita2021/cubaneType/gap/enum-Oh-cube.gap");

LogTo("c:/fujita000/fujita2021/cubaneType/gap/enum-Oh-cubelog.txt") ;

Read("c:/fujita000/fujita2021/cubaneType/gap/CICFgenCC.gapfunc"); \#Loading of CICFgenCC.gapfunc

b_1 := Indeterminate (Rationals, "b_1"); b_2 := Indeterminate (Rationals, "b_2");

$\mathrm{b}_{-} 3:=$ Indeterminate (Rationals, "b_3"); b_4 := Indeterminate (Rationals, "b_4");

b_5 := Indeterminate (Rationals, "b_5"); b_6 := Indeterminate (Rationals, "b_6");

b_7 := Indeterminate (Rationals, "b_7"); b_8 := Indeterminate (Rationals, "b_8");

a_1 := Indeterminate (Rationals, "a_1"); a_2 := Indeterminate (Rationals, "a_2");

$a_{-} 3:=$ Indeterminate (Rationals, "a_3"); a_4 := Indeterminate (Rationals, "a_4");

a_5 := Indeterminate (Rationals, "a_5"); a_6 := Indeterminate (Rationals, "a_6");

a_7 := Indeterminate (Rationals, "a_7"); a_8 := Indeterminate (Rationals, "a_8");

$c_{-} 2:=$ Indeterminate (Rationals, "c_2"); c_4 := Indeterminate (Rationals, "c_4");

c_6 := Indeterminate (Rationals, "c_6"); c_8 := Indeterminate (Rationals, "c_8");

$\operatorname{PCICF}:=[]$

PCICF [1] : = 1/48*b_1 8-1/8*c_2^2*a_1 4-1/12*b_1^2*b_3^2-3/16*b_2 4-1/12*c_2 4 4+1/4*a_1^2*a_3^2+1/8*a_2^4

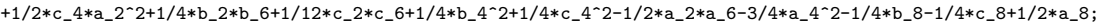
PCICF [2] : : $1 / 8 * b_{-} 2 \sim 4-1 / 8 * a_{-} 2 \sim 4-3 / 8 * b_{-} 4 \sim 2-3 / 8 * c_{-} 4 \sim 2+3 / 4 * a_{-} 4 \sim 2+1 / 4 * b_{-} 8+3 / 4 * c_{-} 8-a_{-} 8$;

PCICF [3] : = 1/4*b_2 4-1/2*c_4*a_2^2-1/2*b_2*b_6-1/4*b_4^2+1/2*a_2*a_6+1/2*a_4^2+1/2*b_8-1/2*a_8;

PCICF $[4]:=1 / 8 * c_{-} 2 \sim 4-1 / 4 * c_{-} 4 * a_{-} 2 \sim 2-3 / 8 * c_{-} 4 \sim 2+1 / 4 * a_{-} 4 \sim 2+1 / 4 * c_{-} 8$;

PCICF [5] : : $1 / 4 * c_{-} 2 \sim 2 * a_{-} 1 \sim 4-1 / 2 * a_{-} 1 \sim 2 * a_{-} 3 \sim 2-1 / 4 * a_{-} 2 \sim 4-1 / 2 * c_{-} 4 * a_{-} 2 \sim 2+1 / 2 * a_{-} 2 * a_{-} 6+a_{-} 4 \sim 2-1 / 2 * a \_8$;

$\operatorname{PCICF}[6]:=1 / 24 * c_{-} \_\sim 4-1 / 4 * c_{-} 4 * a_{-} 22^{\sim} 2-1 / 6 * c_{-} 2 * c_{-} 6-1 / 8 * c_{-} 4 \sim 2+1 / 2 * a_{-} 2 * a_{-} 6+1 / 4 * a_{-} 4 \sim 2+1 / 4 * c_{-} 8-1 / 2 * a_{-} 8$;

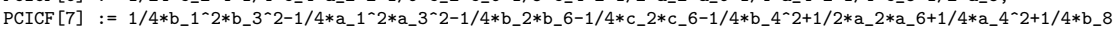
$\hookrightarrow+1 / 4 * c_{-} 8-1 / 2 * a_{-} 8$

PCICF [8] := 1/4*b_4^ $2-1 / 4 * a_{-} 44^{\wedge} 2-1 / 4 * b_{-} 8-1 / 4 * c_{-} 8+1 / 2 * a \_8$;

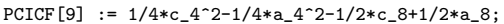

$\operatorname{PCICF}[10]:=0$;

$\operatorname{PCICF}[11]:=1 / 4 * b_{-} 44^{\sim} 2-1 / 4 * a_{-} 44^{\sim} 2-1 / 4 * b_{-} 8-1 / 4 * c_{-} 8+1 / 2 * a \_8$;

$\operatorname{PCICF}[12]:=1 / 4 * C_{-} 4^{\wedge} 2-1 / 4 * a_{-} 4^{\wedge} 2-1 / 2 * c_{-} 8+1 / 2 * a_{-} 8$;

$\operatorname{PCICF}[13]:=1 / 4 * \mathrm{a}_{-} 2 \sim 4-3 / 4 * \mathrm{a}_{-} 44^{\sim} 2+1 / 2 * \mathrm{a}_{-} 8$;

PCICF $[14]:=1 / 2 * c_{-} 4 * a_{-} 2 \sim 2-1 / 2 * a_{-} 4{ }^{\sim} 2$;

$\operatorname{PCICF}[15]:=1 / 4 * c_{-} 44^{\sim} 2-1 / 4 * a_{-} 4^{\wedge} 2-1 / 2 * c_{-} 8+1 / 2 * a_{-} 8$

$\operatorname{PCICF}[16]:=1 / 2 * c_{-} 4 * a_{-} 2 \sim 2-a_{-} 2 * a_{-} 6-1 / 2 * a_{-} 4 \sim 2+a_{-} 8 ;$

PCICF [17] : = 1/2*b_2*b_6-1/2*a_2*a_6-1/2*b_8+1/2*a_8;

PCICF [18] := 1/2*a_1 $2 * a_{-} 3{ }^{-} 2-1 / 2 * a_{-} 2 * a_{-} 6-1 / 2 * a_{-} 4 \sim 2+1 / 2 * a_{-} 8$;

PCICF $[19]:=1 / 2 * c_{-} 2 * c_{-} 6-1 / 2 * a_{-} 2 * a_{-} 6-1 / 2 * c_{-} 8+1 / 2 * a_{-} 8$;

$\operatorname{PCICF}[20]:=0$;

PCICF $[21]:=1 / 2 * a_{-} 4 \sim 2-1 / 2 * a_{-} 8$;

$\operatorname{PCICF}[22]:=1 / 2 * C_{-} 8-1 / 2 * a_{-} 8$

$\operatorname{PCICF}[23]:=0$;

PCICF $[24]:=1 / 2 * C_{-} 8-1 / 2 * a_{-} 8$

PCICF [25] :=0;

PCICF [26] := 1/2*a_4^2-1/2*a_8;

PCICF [27] := 1/4*b_4^2-1/4*a_4^2-1/4*b_8-1/4*c_8+1/2*a_8;

PCICF [28] := a_2*a_6-a_8;

PCICF [29] :=0;

PCICF $[30]:=1 / 2 * b_{-} 8-1 / 2 * a_{-} 8 ;$

PCICF $[31]:=1 / 2 * C_{-} 8-1 / 2 * a_{-} 8 ;$

PCICF [32] := 1/2*a_4^2-1/2*a_8;

$\operatorname{PCICF}[33]:=\mathrm{a}_{-} 8$;

$\mathrm{A}:=$ Indeterminate (Rationals, "A"); $\mathrm{B}:=$ Indeterminate (Rationals, "B");

$\mathrm{C}:=$ Indeterminate (Rationals, "C"); $\mathrm{D}:=$ Indeterminate (Rationals, "D");

$\mathrm{V}:=$ Indeterminate (Rationals, "V"); W := Indeterminate (Rationals, "W");

$\mathrm{p}:=$ Indeterminate (Rationals, "p"); $\mathrm{P}:=$ Indeterminate (Rationals, "P");

$\mathrm{q}:=$ Indeterminate (Rationals, "q"); $\mathrm{Q}:=$ Indeterminate (Rationals, "Q");

aa_1 : $=\mathrm{A}+\mathrm{B}+\mathrm{C}+\mathrm{D}+\mathrm{V}+\mathrm{W}$

aa_2 : $=A^{\wedge} 2+B^{\wedge} 2+C^{\wedge} 2+D^{\wedge} 2+V^{\wedge} 2+W^{\wedge} 2$

aa_3 $:=A^{\wedge} 3+B^{\wedge} 3+C^{\wedge} 3+D^{\wedge} 3+V^{\wedge} 3+W^{\wedge} 3$

aa_4 $:=A^{\wedge} 4+B^{\wedge} 4+C^{\wedge} 4+D^{\wedge} 4+V^{\wedge} 4+W^{\wedge} 4$

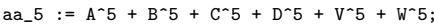




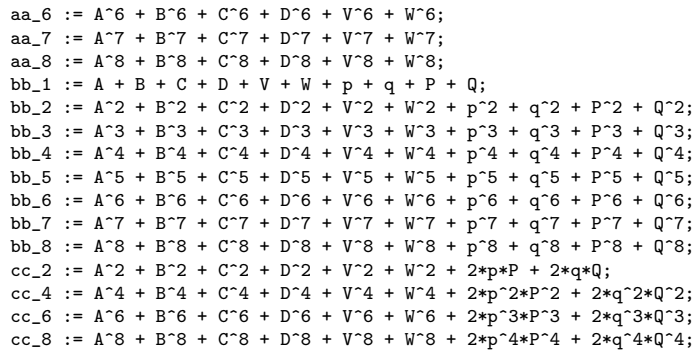


$\left[a_{-} 1, a_{-} 2, a_{-} 3, a_{-} 4, a_{-} 5, a_{-} 6, a_{-} 7, a_{-} 8\right.$,

$\left.b_{-} 1, b_{-} 2, b_{-} 3, b_{-} 4, b_{-} 5, b_{-} 6, b_{-} 7, b_{-} 8, c_{-} 2, c_{-} 4, c_{-} 6, c_{-} 8\right]$,

[aa_1, aa_2, aa_3, aa_4, aa_5, aa_6, aa_7, aa_8,

bb_1, bb_2, bb_3, bb_4, bb_5, bb_6, bb_7, bb_8, cc_2, cc_4, cc_6, cc_8]); ;

f_12:= Value (PCICF [12],

$\left[a_{-} 1, a_{-} 2, a_{-} 3, a_{-} 4, a_{-} 5, a_{-} 6, a_{-} 7, a_{-} 8\right.$,

b_1, b_2, b_3, b_4, b_5, b_6, b_7, b_8, c_2 $\left.c_{-} 4, c_{-} 6, c_{-} 8\right]$,

[aa_1, aa_2, aa_3, aа_ 4 , aа_ 5, aa_6, aa_7, aа_ 8 ,

bb_1, bb_2 $\left.\left.b_{-} b_{-} 3, b_{-} 4, b_{-} 5, b_{-} 6, b_{-} 7, b_{-} 8, c_{-} 2, c_{-} 4, c_{-} 6, c_{-} 8\right]\right)$; ;

(omitted)

f_29:=0; ;

f_30:= Value (PCICF $[30]$,

$\left[a_{-} 1, a_{-} 2, a_{-} 3, a_{-} 4, a_{-} 5, a_{-} 6, a_{-} 7, a_{-} 8\right.$,

b_1, b_2, b_3, b_4, b_5, b_6, b_7, b_8, c_2 , c_4, c_6, c_8],

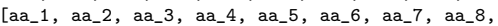

bb_1, bb_2, bb_3, bb_4, bb_5, bb_6, bb_7, bb_8, cc_2

f_31:= Value (PCICF [31],

$\left[a_{-} 1, a_{-} 2, a_{-} 3, a_{-} 4, a_{-} 5, a_{-} 6, a_{-} 7, a_{-} 8\right.$,

b_1, b_2, b_3, b_4, b_5, b_6, b_7, b_8, c_2 , c_4 , c_6, c_8],

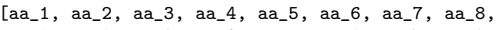

bb_1 1 b__ $\left.\left.2, b_{-} 3, b_{-} 4, b_{-} 5, b_{-} 6, b_{-} 7, b_{-} 8, c_{-} 2, c_{-} 4, c_{-} 6, c_{-} 8\right]\right)$; ;

f_32:= Value $($ PCICF $[32]$,

[a_1, a_2, a_3, a_4, a_5, a_6, a_7, a_8,

b_1, b_2, b_3, b_4, b_5, b_6, b_7, b_8, c_2 $\left.c_{-} 4, c_{-} 6, c_{-} 8\right]$,

[aа_1, aа_2, aа_3, aа_4, aа_5, aа_6, aа_ 7 , aа_8,

bb_1, bb_2, bb_3, bb_4, bb_5, bb_6, bb_7, bb_8, cc_2 $\left., \mathrm{cc}_{-} 4, \mathrm{cc}_{-} 6, \mathrm{cc}_{-} 8 \mathrm{~d}\right)$; ;

f_33:= Value (PCICF [33],

$\left[a_{-} 1, a_{-} 2, a_{-} 3, a_{-} 4, a_{-} 5, a_{-} 6, a_{-} 7, a_{-} 8\right.$,

b_1, b_2, b_3, b_4, b_5, b_6, b_7, b_8, c_2 , c_4, c_6, c_8],

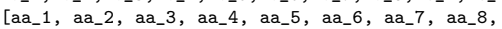

bb_1, bb_2, bb_3, bb_ $\left.\left.4, b_{-} 5, b_{-} 6, b_{-} 7, b_{-} 8, c_{-} 2, c_{-} 4, c_{-} 6, c_{-} 8\right]\right)$; ;

list_partitions :=[];

calcCoeffGencube := function(list_partitions)

local list_ligand_L, I_pp;

list_ligand_L : $=[A, B, C, D, V, W, p, P, q, Q]$;

$1_{\text {_pp }}:=$ list_partitions;

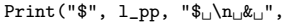

calcCoeffGen(f_1, list_ligand_L, list_partitions), "ப\&ப",

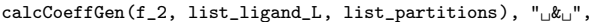
calcCoeffGen(f_3, list_ligand_L, list_partitions), "ப\& $\sqcup "$, calcCoeffGen (f_4, list_ligand_L, list_partitions), "ப\&४",

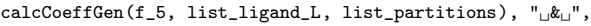
calcCoeffGen (f_6, list_ligand_L, list_partitions), "ப\&ப", calcCoeffGen(f_7, list_ligand_L, list_partitions), "ப\&४", calcCoeffGen(f_8, list_ligand_L, list_partitions), "ப\&ப", calcCoeffGen(f_9, list_ligand_L, list_partitions), "ப\&ப",

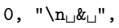

calcCoeffGen (f_11, list_ligand_L, list_partitions), "ப\& $\sqcup$ ", calcCoeffGen(f_12, list_ligand_L, list_partitions), "ப\&ப",

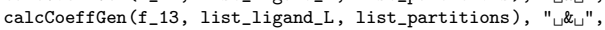
calcCoeffGen(f_14, list_ligand_L, list_partitions), "५\&५", calcCoeffGen(f_15, list_ligand_L, list_partitions), "ப\&৬", calcCoeffGen(f_16, list_ligand_L, list_partitions), "ப\& $\sqcup "$, calcCoeffGen(f_17, list_ligand_L, list_partitions), "ப\&ப", calcCoeffGen(f_18, list_ligand_L, list_partitions), " $\& \sqcup "$ ", calcCoeffGen(f_19, list_ligand_L, list_partitions), "८\&" , 0 , "\} \mathrm { n } _ { \sqcup } \& \sqcup "

calcCoeffGen(f_21, list_ligand_L, list_partitions), "ப\&४",

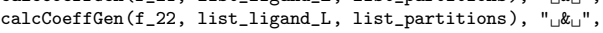
0 , "ப\&५",

calcCoeffGen(f_24, list_ligand_L, list_partitions), "ப\&ப", 0 , "५४५",

calcCoeffGen(f_26, list_ligand_L, list_partitions), "ப\&ப", calcCoeffGen(f_27, list_ligand_L, list_partitions), "ப\&ப", calcCoeffGen(f_28, list_ligand_L, list_partitions), "८\&し", 
0 , "४\&",

calcCoeffGen (f_30, list_ligand_L, list_partitions), "५\&ப", calcCoeffGen (f_31, list_ligand_L, list_partitions), "ப\&ப", calcCoeffGen (f_32, list_ligand_L, list_partitions), "৬\&ப",

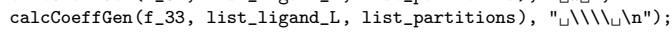
end;

calcCoeffGencube $([8,0,0,0,0,0,0,0,0,0])$; calcCoeffGencube $([7,1,0,0,0,0,0,0,0,0])$; calcCoeffGencube $([6,2,0,0,0,0,0,0,0,0])$; calcCoeffGencube $([6,1,1,0,0,0,0,0,0,0])$; calcCoeffGencube $([5,3,0,0,0,0,0,0,0,0])$; calcCoeffGencube $([5,2,1,0,0,0,0,0,0,0])$; calcCoeffGencube $([5,1,1,1,0,0,0,0,0,0])$; calcCoeffGencube $([4,4,0,0,0,0,0,0,0,0])$; calcCoeffGencube $([4,3,1,0,0,0,0,0,0,0])$; calcCoeffGencube $([4,2,2,0,0,0,0,0,0,0])$; calcCoeffGencube $([4,2,1,1,0,0,0,0,0,0])$; calcCoeffGencube $([4,1,1,1,1,0,0,0,0,0])$; calcCoeffGencube $([3,3,2,0,0,0,0,0,0,0])$; calcCoeffGencube $([3,3,1,1,0,0,0,0,0,0])$; calcCoeffGencube $([3,2,2,1,0,0,0,0,0,0])$; calcCoeffGencube $([3,2,1,1,1,0,0,0,0,0])$; calcCoeffGencube $([2,2,2,2,0,0,0,0,0,0])$; calcCoeffGencube $([2,2,2,1,1,0,0,0,0,0])$; calcCoeffGencube $([2,2,1,1,1,1,0,0,0,0])$;

(omitted)

calcCoeffGencube $([1,1,1,1,1,1,1,1,0,0])$; calcCoeffGencube $([1,1,1,1,1,0,1,1,1,0])$; calcCoeffGencube $([1,1,1,1,0,0,1,1,1,1])$; 


\section{Appendix B}

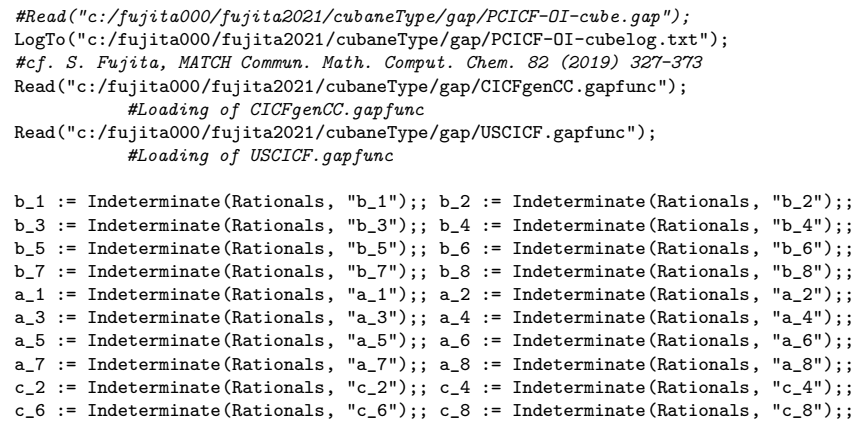

\#Read("c:/fujita000/fujita2021/cubaneType/gap/PCICF-OI-cube.gap"); LogTo ("c:/fujita000/fujita2021/cubaneType/gap/PCICF-OI-cubelog.txt"); \#cf. S. Fujita, MATCH Commun. Math. Comput. Chem. 82 (2019) 327-373 Read("c:/fujita000/fujita2021/cubaneType/gap/CICFgenCC.gapfunc"); \#Loading of CICFgenCC.gapfunc

Read ("c:/fujita000/fujita2021/cubaneType/gap/USCICF.gapfunc") ; \#Loading of USCICF.gapfunc

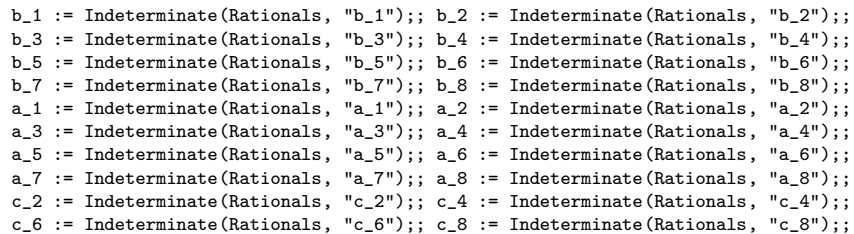

O_cube $:=\operatorname{Group}([(1,2,3,4)(5,6,7,8),(2,4,5)(3,8,6)])$; ;

CICF_O_cube := CalcConjClassCICF $\left(0 \_c u b e, 8,8\right)$;

Display (CICF_O_cube);

Display ("\#\#\#\#\#\#\#\#\#\#\#\#\#\#\#\#\#\#\#\#\#\#\#\#\#\#\#\#--03") ;

OI_cube := $\operatorname{Group}([(1,2,3,4)(5,6,7,8),(2,4,5)(3,8,6),(9,10)])$; ;

Size(OI_cube);

CICF_OI_cube := CalcConjClassCICF (OI_cube, 8, 10);

Display ("CICF_OI_cube=");

Display (CICF_OI_cube);

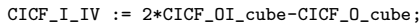

Display ("CICF_I_IV $:="$ ");

Display (CICF_I_IV);

CICF_II_III_V $:=$ CICF_O_cube-CICF_OI_cube

Display ("CICF_II_III_V

Display (CICF_II_III_V);

\#tom_OI_cube := TableOfMarks (OI_cube);

\#Display(tom_OI_cube);

\#

\#gen : $=[] ; ;$

\#for $i$ in [1..33] do

\#gen [i] := RepresentativeTom(tom_OI_cube, $i$ );

\#isplay (gen $[i])$;

\#od;

gen $:=[] ;$;

gen $[1]:=[] ; ; \# 1$

$\operatorname{gen}[2]:=[(1,3)(2,4)(5,7)(6,8)] ; ; \# 2$

gen $[3]:=[(1,7)(2,6)(3,5)(4,8)] ; ; \# 3$

gen $[4]:=[(9,10)] ; ; \# 4$

gen $[5]:=[(1,3)(2,4)(5,7)(6,8)(9,10)] ; ; \# 5$

gen $[6]:=[(1,7)(2,6)(3,5)(4,8)(9,10)] ; ; \# 6$

gen $[7]:=[(2,5,4)(3,6,8)] ; ; \# 7$

gen $[8]:=[(1,8)(2,7)(3,6)(4,5),(1,3)(2,4)(5,7)(6,8)] ; ; \# 8$

gen $[9]:=[(9,10),(1,3)(2,4)(5,7)(6,8)] ; ; \# 9$

gen $[10]:=[(1,8)(2,7)(3,6)(4,5)(9,10),(1,3)(2,4)(5,7)(6,8)] ; ; \# 10$

$\operatorname{gen}[11]:=[(1,2,3,4)(5,6,7,8)(9,10),(1,3)(2,4)(5,7)(6,8)] ; ; \# 11$

gen $[12]:=[(1,7)(2,6)(3,5)(4,8)(9,10),(1,3)(2,4)(5,7)(6,8)] ; ; \# 12$

$\operatorname{gen}[13]:=[(1,2,3,4)(5,6,7,8),(1,3)(2,4)(5,7)(6,8)] ; ; \# 13$

gen $[14]:=[(1,7)(2,6)(3,5)(4,8),(1,3)(2,4)(5,7)(6,8)] ; ; \# 14$

gen $[15]:=[(9,10),(1,7)(2,6)(3,5)(4,8)] ; ; \# 15$

$\operatorname{gen}[16]:=[(1,3)(2,4)(5,7)(6,8)(9,10),(1,7)(2,6)(3,5)(4,8)] ; ; \# 16$

gen $[17]:=[(9,10),(2,5,4)(3,6,8)] ; ; \# 17$

gen $[18]:=[(1,7)(2,6)(3,5)(4,8),(2,5,4)(3,6,8)] ; ; \# 18$

gen $[19]:=[(1,7)(2,6)(3,5)(4,8)(9,10),(2,5,4)(3,6,8)] ; ; \# 19$

$\operatorname{gen}[20]:=[(1,8)(2,7)(3,6)(4,5),(1,3)(2,4)(5,7)(6,8),(1,2,3,4)(5,6,7,8)] ;$; \#20 
$\operatorname{gen}[21]:=[(1,8)(2,7)(3,6)(4,5),(1,3)(2,4)(5,7)(6,8),(1,2,3,4)(5,6,7,8)(9,10)] ; ; \# 21$ $\operatorname{gen}[22]:=[(9,10),(1,2,3,4)(5,6,7,8),(1,3)(2,4)(5,7)(6,8)] ; ; \# 22$

gen $[23]:=[(9,10),(1,7)(2,6)(3,5)(4,8),(1,3)(2,4)(5,7)(6,8)] ; ; \# 23$

gen $[24]:=[(1,8)(2,7)(3,6)(4,5),(1,3)(2,4)(5,7)(6,8),(9,10)] ; ; \# 24$

gen $[25]:=[(1,8)(2,7)(3,6)(4,5)(9,10),(1,7)(2,6)(3,5)(4,8),(1,3)(2,4)(5,7)(6,8)] ; ; \# 25$

gen [26] := $[(1,8)(2,7)(3,6)(4,5)(9,10),(1,2,3,4)(5,6,7,8),(1,3)(2,4)(5,7)(6,8)] ; ; \# 26$

$\operatorname{gen}[27]:=[(9,10),(1,7)(2,6)(3,5)(4,8),(2,5,4)(3,6,8)] ; ; \# 27$

$\operatorname{gen}[28]:=[(1,8)(2,7)(3,6)(4,5),(1,3)(2,4)(5,7)(6,8),(2,5,4)(3,6,8)] ; ; \# 28$

gen $[29]:=[(1,8)(2,7)(3,6)(4,5),(1,3)(2,4)(5,7)(6,8),(9,10),(1,2,3,4)(5,6,7,8)] ; ; \# 29$

$\operatorname{gen}[30]:=[(1,8)(2,7)(3,6)(4,5),(1,3)(2,4)(5,7)(6,8),(2,5,4)(3,6,8),(1,2,3,4)(5,6,7,8)] ; ; \# 30$

$\operatorname{gen}[31]:=[(1,8)(2,7)(3,6)(4,5),(1,3)(2,4)(5,7)(6,8),(2,5,4)(3,6,8),(9,10)] ; ; \# 31$

gen $[32]:=[(1,8)(2,7)(3,6)(4,5),(1,3)(2,4)(5,7)(6,8),(2,5,4)(3,6,8)$,

$(1,2,3,4)(5,6,7,8)(9,10)] ; ; \# 32$

$\operatorname{gen}[33]:=[(1,2,3,4)(5,6,7,8),(2,4,5)(3,8,6),(9,10)] ; ; \# 33$

\#mark table sorted for USCI table

MarkTableOI_cube := MarkTableforUSCI (OI_cube,0_cube,33,gen, 8,10) ; ;

Display (MarkTableOI_cube);

USCITableOI_cube := constructUSCITable (OI_cube,0_cube, 33, gen, 8,10);

Display ("\#\#USCI-CF $\mathrm{table}_{\sqcup}$ (USCITableOI_cube) $\sqcup: "$ );

Display (USCITableOI_cube);

\#Matrix form of mark table

Matrix_tomOI_cube := MatTom(MarkTableOI_cube);

Display (Matrix_tomOI_cube);

Display ("\#ixed $\sqcup$ point $\sqcup$ vector for $_{\sqcup}$ cube") );

FPVcube := calculateFPvector (OI_cube,0_cube,33, gen,8,10); ;

Display (FPVcube);

Display ("\#)ultiplicity of $_{\sqcup}$ Orbits");

orbit_OI_cube := FPVcube * Inverse (Matrix_tomOI_cube);

Display (orbit_OI_cube);

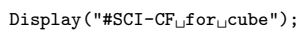

1_SCICF_OI_cube := constructSCICF (OI_cube,O_cube, Matrix_tomOI_cube,USCITableOI_cube,FPVcube); ;

Display (1_SCICF_OI_cube);

Display ("\#list of $_{\sqcup} \mathrm{PCI}-\mathrm{CF} \mathrm{s}_{\sqcup}$ for $_{\sqcup}$ cube");

1_PCICF_OI_cube := 1_SCICF_OI_cube * Inverse (Matrix_tomOI_cube); ;

Display (1_PCICF_OI_cube);

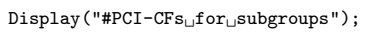

for $i$ in $[1 . .33]$ do

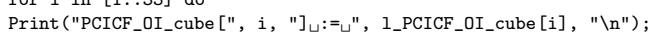

od;

$\log \mathrm{To}()$; 